University of Louisville

ThinkIR: The University of Louisville's Institutional Repository

Electronic Theses and Dissertations

$12-2016$

\title{
Distress, circadian rhythms, immunity, and survival in presurgical breast cancer patients.
}

Whitney Nicole Rebholz

University of Louisville

Follow this and additional works at: https://ir.library.louisville.edu/etd

Part of the Health Psychology Commons

\section{Recommended Citation}

Rebholz, Whitney Nicole, "Distress, circadian rhythms, immunity, and survival in presurgical breast cancer patients." (2016). Electronic Theses and Dissertations. Paper 2610.

https://doi.org/10.18297/etd/2610

This Doctoral Dissertation is brought to you for free and open access by ThinkIR: The University of Louisville's Institutional Repository. It has been accepted for inclusion in Electronic Theses and Dissertations by an authorized administrator of ThinkIR: The University of Louisville's Institutional Repository. This title appears here courtesy of the author, who has retained all other copyrights. For more information, please contact thinkir@louisville.edu. 


\title{
DISTRESS, CIRCADIAN RHYTHMS, IMMUNITY, AND SURVIVAL
} IN PRESURGICAL BREAST CANCER PATIENTS

\author{
By \\ Whitney Nicole Rebholz \\ B.A., University of Louisville, 2011 \\ M.A., University of Louisville, 2013

\begin{abstract}
A Dissertation
Submitted to the Faculty of the

College of Arts and Sciences of the University of Louisville

in Partial Fulfillment of the Requirements

for the Degree of
\end{abstract} \\ Doctor of Philosophy \\ in Clinical Psychology \\ Department of Psychological and Brain Sciences \\ University of Louisville \\ Louisville, Kentucky
}

December 2016 
Copyright 2016 by Whitney Nicole Rebholz

All rights reserved. 

DISTRESS, CIRCADIAN RHYTHMS, IMMUNITY, AND SURVIVAL IN PRESURGICAL BREAST CANCER PATIENTS

\author{
By \\ Whitney Nicole Rebholz \\ B.A., University of Louisville, 2011 \\ M.A., University of Louisville, 2013 \\ A Dissertation Approved on
}

October 10, 2016

by the following Dissertation Committee:

Dissertation Director
Sandra E. Sephton, Ph.D.

Paul Salmon, Ph.D.

Barbara Stetson, Ph.D.

Tamara Newton, Ph.D.

Elizabeth Cash, Ph.D. 


\section{DEDICATION}

This dissertation is dedicated to my Nanny and PaPa, Barbara and George Gesler, who taught me from a young age to never let anyone or anything keep me from my dreams, no matter how lofty. Their love and encouragement was never-ending, and for that I am eternally grateful. 


\section{ACKNOWLEDGEMENTS}

This dissertation would not have been possible without the endless support and guidance from Dr. Sandra Sephton. Her continuous support and nurturance, which began during my years as an undergraduate research assistant, have helped in shaping me personally and professionally. I would not be the Scientist-Practitioner that I am today without her steadfast commitment to my growth and development. For that, I am forever grateful. I am also thankful for the support and guidance from my friend and mentor, Dr. Elizabeth Cash, whose encouragement helped me through the most difficult of times throughout graduate school. I would like to thank those in the Biobehavioral Research Laboratory who made this study possible. While I was not a member of the lab during the recruitment of the study, I am very appreciative of the dedication, determination, and attention that went into data collection and processing, without which this dissertation would not be possible.

I would like to thank my family, particularly my parents, Rebecca and G. David Gesler, who taught me the value of hard work and to never stop learning. Their unconditional love, encouragement, and support helped me to become the person that I am today. Lastly, I would like to thank my husband, Luke Rebholz, who has stood by my side since I began this journey and has never wavered in his love and support of me.

Funding for this study was provided by a University of Louisville Research Grant, which funded a first wave of recruitment and collection of psychometric, actigraphy, 
saliva, and blood data. A Stanford Cancer Center Grant funded a second round of recruitment, as well as the shipment and assay of all immune outcomes. 


\section{ABSTRACT \\ DISTRESS, CIRCADIAN RHYTHMS, IMMUNITY, AND SURVIVAL IN PRESURGICAL BREAST CANCER PATIENTS

\author{
Whitney Nicole Rebholz
}

October 10, 2016

Women with breast cancer experience significant stress related to diagnosis and treatment, which may negatively affect physiology (e.g., circadian, endocrine, and immune) and contribute to cancer progression. Demographic (e.g., ethnic minority) and neighborhood factors can contribute to cancer-related stress. African American women with breast cancer experience more adverse disease pathology and earlier mortality; however, factors that explain these disparities rarely have been explored. It is hypothesized that psychosocial stressors (i.e., ethnicity, neighborhood stress, and cancerrelated stress) will be related to one another. Associations of psychosocial stress with physiological disruption are posited with regard to circadian, endocrine, and immune function. It is also hypothesized that psychosocial stress and physiological disruption will predict earlier cancer recurrence/mortality.

Presurgical breast cancer patients $(\mathrm{N}=57)$ reported on demographics and cancerrelated stress and provided three-day home-based collection of rest/activity rhythms and salivary cortisol. Neighborhood stress data were obtained via the 2010 Census. Immune parameters were assessed in serum samples. Progression-free survival status was obtained at a median of seven years post-enrollment. 
Bivariate correlations examined relationships between psychosocial stressors. Hierarchical linear regressions adjusted for age, cancer stage, and income in tests of associations between psychosocial stress and physiological disruption. Cox proportional hazards analyses tested the prognostic value of psychosocial stress and physiological disruption on progression-free survival from initial diagnosis and study entry.

African Americans had higher neighborhood stress compared to non-Hispanic White patients. Patients with greater neighborhood stress had greater overall cortisol secretion $\left(\Delta \mathrm{R}^{2}=.246, \mathrm{p}=.001\right)$. Patients reporting greater cancer-related stress had accentuated diurnal cortisol rhythms $\left(\Delta \mathrm{R}^{2}=.084, \mathrm{p}=.047\right)$. Exploratory post hoc analyses revealed this finding was likely driven by avoidance of cancer-related thoughts. Psychosocial stress was not significantly related to circadian or immune disruption, nor was psychosocial stress or physiological disruption predictive of progression-free survival.

Improved resources within disadvantaged neighborhoods may help counter the effects of neighborhood stress on cortisol disruption by reducing stress and improving health outcomes. Interestingly, during the acutely stressful presurgical period, avoidance appears to serve as an adaptive coping strategy. Therefore, the presurgical period may be the opportune time to introduce psychosocial interventions to combat challenges throughout one's cancer journey. 
ACKNOWLEDGMENTS ..................................................................................... iv

ABSTRACT .......................................................................................................... vi

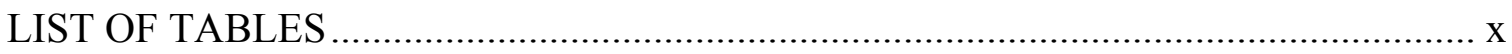

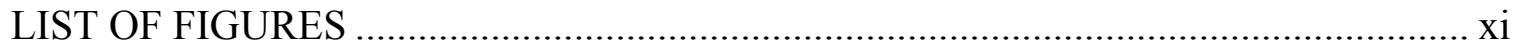

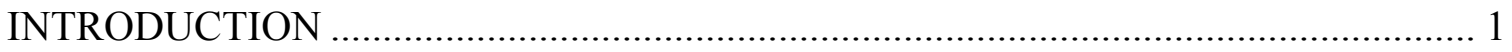

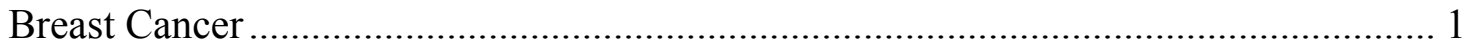

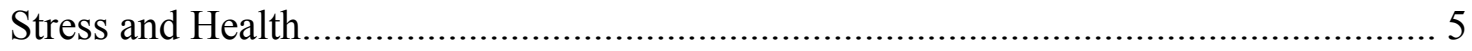

Stress Related to Ethnicity ................................................................................... 6

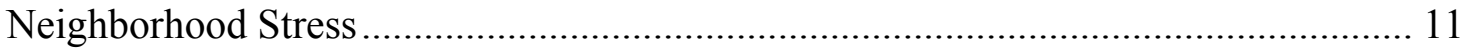

Cancer-Related Stress ........................................................................................ 12

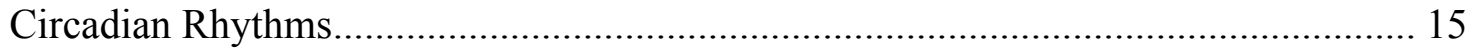

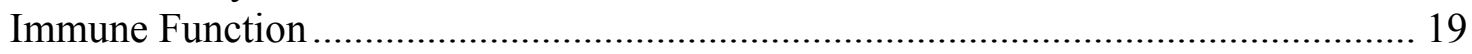

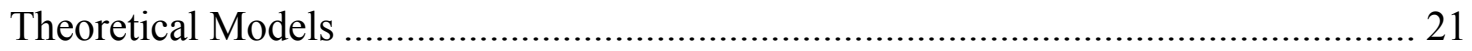

Proposed Model ................................................................................................ 22

Ethnicity and Physiological Disruption (Figure 1 Arrow A) ........................................ 23

Neighborhood Stress and Physiological Disruption (Figure 1 Arrow A) ..................... 27

Cancer-Related Stress and Physiological Disruption (Figure 1 Arrow A) ................... 30

Psychosocial Stress and Medical Outcomes of Cancer (Figure 1 Arrow B) ................ 32

Physiological Disruption and Medical Outcomes of Cancer (Figure 1 Arrow C)........ 37

Summary and Hypotheses...................................................................................... 42

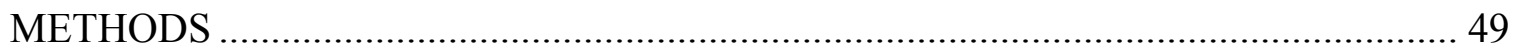

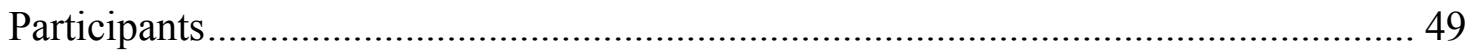

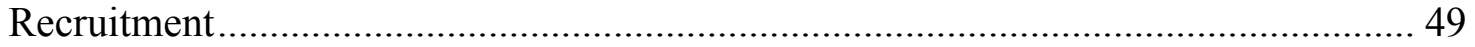

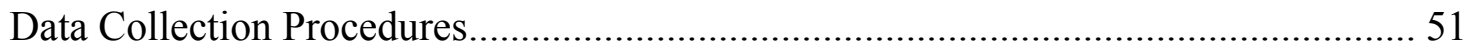

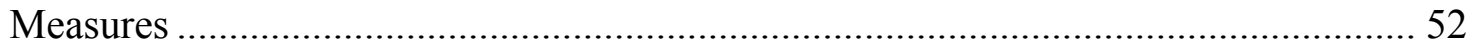

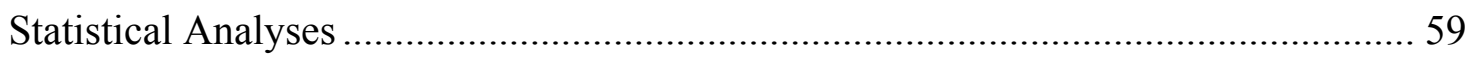

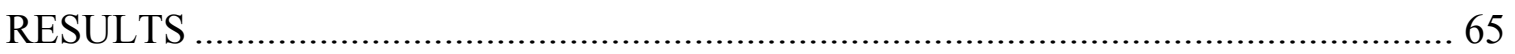

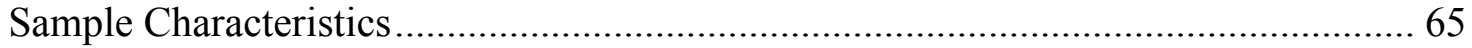

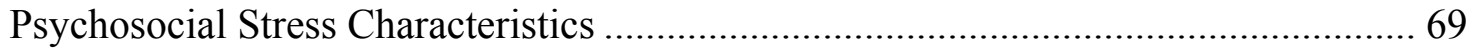

Physiological Disruption Characteristics ................................................................ 71

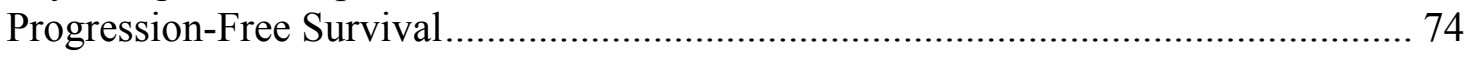

Principal Components Analysis of Neighborhood Stress ............................................ 74 
Primary Analyses ............................................................................................ 76

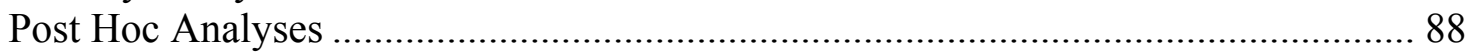

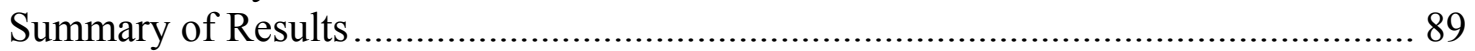

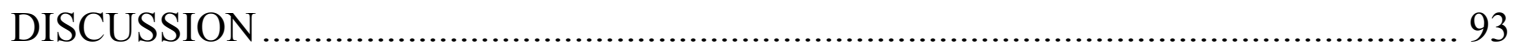

Associations Between Measures of Psychosocial Stress: Ethnicity was correlated with neighborhood stress, but not cancer-related stress. Neighborhood stress was not correlated with cancer-related stress (Figure 2) ................................................. 96

Associations Between Psychosocial Stress and Physiological Disruption .................. 98

No predictors of progression-free survival emerged. .......................................... 109

Low-income patients had poorer circadian coordination. ..................................... 112

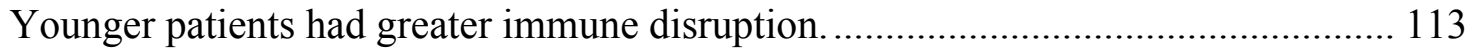

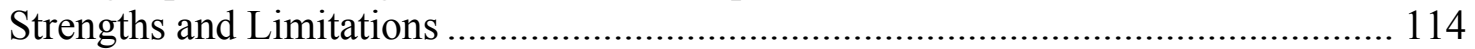

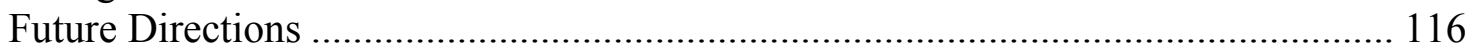

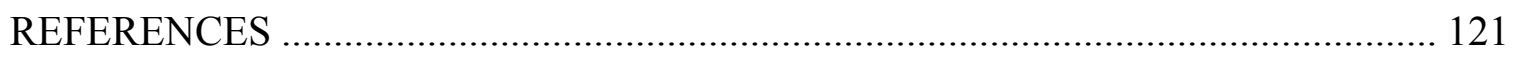

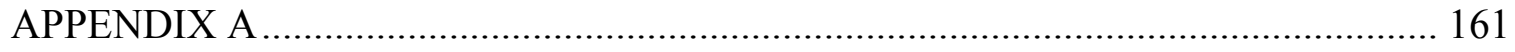

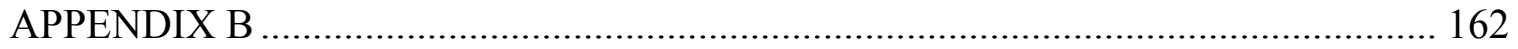

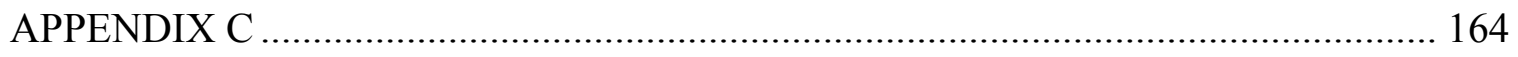

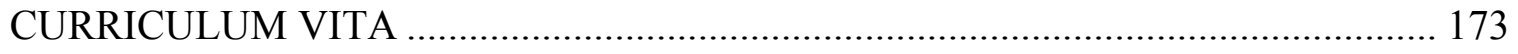




\section{LIST OF TABLES}

TABLE PAGE

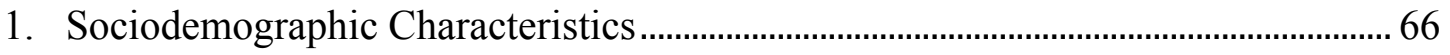

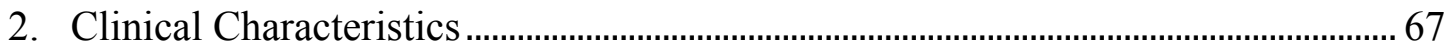

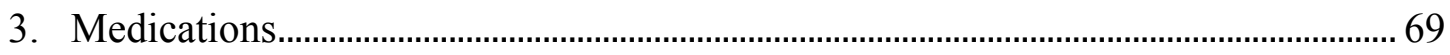

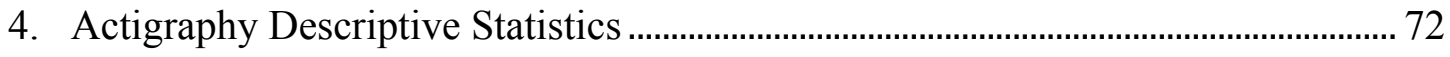

5. Diurnal Cortisol Descriptive Statistics ……….............................................................. 72

6. Immune Function Descriptive Statistics .................................................................... 73

7. Progression-Free Survival Descriptive Statistics ........................................................... 74

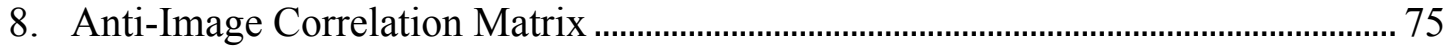

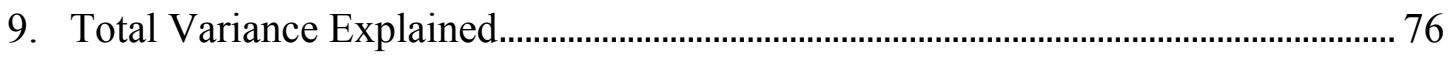

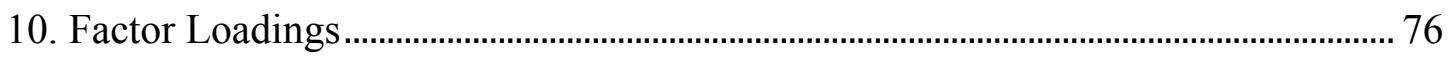

11. Spearman's rho correlation matrix for hypothesis 1. ................................................... 77

12. Significant results for hypothesis 2 A.2: Hierarchical regression analyses entered neighborhood stress as a predictor of endocrine disruption. ..................................... 81

13. Summary of results for hypothesis 2A.3: Hierarchical regression analyses entered cancer-related stress as a predictor of endocrine disruption. 85

14. Summary of results for post hoc analyses: Hierarchical regression analyses entered cancer-related stress subscales as a predictor of diurnal cortisol slope. ... 89 


\section{LIST OF FIGURES}

\section{FIGURE}

PAGE

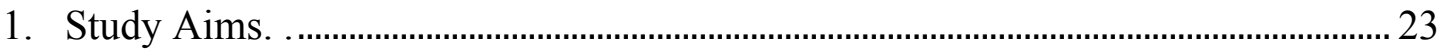

2. Aim 1, associations between psychosocial stress measures....................................... 43

3. Hypothesis 2A.1, associations of ethnicity with physiological disruption. ............ 44

4. Hypothesis 2A.2, associations of neighborhood stress with physiological

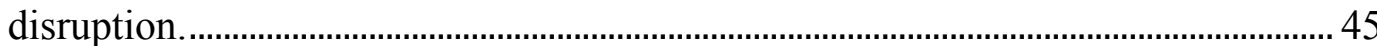

5. Hypothesis 2A.3, associations of cancer-related stress with physiological disruption.

6. Hypothesis 2B, associations of psychosocial stress with medical outcomes of cancer (progression-free survival time).

7. Hypothesis $2 \mathrm{C}$, associations of physiological disruption with medical outcomes of cancer (progression-free survival time)

8. Mean with $95 \%$ confidence interval diurnal salivary cortisol values over the threeday data collection period.

9. Significant associations with control variables for associations of ethnicity with physiological disruption.

10. Significant association between neighborhood stress and endocrine disruption (i.e., diurnal mean).

11. Significant associations with control variables for associations of neighborhood stress with physiological disruption.

12. Significant association between cancer-related stress and endocrine disruption (i.e., diurnal slope).

13. Mean and $95 \%$ confidence interval raw diurnal cortisol values with median split IES summary scores.

14. Significant associations with control variables for associations of cancer-related stress with physiological disruption. 
15. Mean and $95 \%$ confidence interval raw diurnal cortisol values with median split

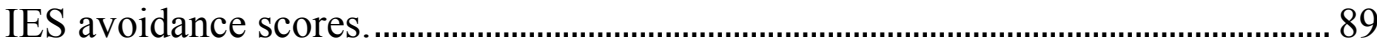




\section{INTRODUCTION}

\section{Breast Cancer}

Incidence, risk, and mortality. Breast cancer is the most common cancer diagnosed in women and accounts for approximately $29 \%$ of new female cancer diagnoses (Siegel, Ma, Zou, \& Jemal, 2014). In the 1980s, rates of breast cancer increased dramatically, primarily due to better detection through utilization of mammography (DeSantis, Ma, Bryan, \& Jemal, 2014). Increases in rates of obesity and use of menopausal hormones, both of which are breast cancer risk factors, partly explain a small increase in incidence rates in the late 1990s (DeSantis et al., 2014). In the early 2000s, the rates of breast cancer incidence dropped drastically, most likely due to a decrease in the use of hormone replacement therapy following a Women's Health Initiative publication (DeSantis et al., 2014). The current risk of being diagnosed with breast cancer is 1 in 8 for women living in the United States (DeSantis et al., 2014). Incidence rates of breast cancer have remained stable over the past decade (Siegel et al., 2014).

Breast cancer is the second most common cause of death from cancer among women, accounting for $15 \%$ of cancer deaths in women (Siegel et al., 2014). Among women aged 20 to 59, breast cancer is the leading cause of cancer death (Siegel et al., 2014). However, rates of mortality from breast cancer have been declining, particularly due to improvements in detection and treatment (DeSantis et al., 2014). 
Breast cancer types. There are different types of breast cancer, which are classified based on the origin and spread of cancer. Ductal carcinoma in situ (DCIS) is noninvasive carcinoma, meaning cancer occurs within the breast ducts and has not spread beyond the duct of origin (Van Goethem et al., 2006). Invasive breast carcinoma indicates that cancer has spread beyond the site of origin (e.g., duct or lobule) and into the surrounding breast tissue (Andersson, 2005). Invasive carcinomas often develop from DCIS. When cancer has spread to distant tissue and organs, it is considered metastatic disease.

Breast tumors can be classified as a carcinoma or sarcoma. Carcinoma is the most common type of breast cancer, which indicates that the cancer originated in the epithelial cell lining of breast tissue (Andersson, 2005). Sarcomas, which arise from the mesenchymal tissue of the breast (Adem, Reynolds, Ingle, \& Nascimento, 2004), account for less than one percent of breast cancer diagnoses (Pandey, Mathew, Abraham, \& Rajan, 2004).

Disease staging. Breast cancer is staged in order to measure the extent of the disease. The tumor-node-metastasis (TNM) staging system is widely used and is based on the size of the tumor, the presence of metastasis to lymph nodes, and the presence of distant metastases (Singletary et al., 2002). Stages of breast cancer range from 0 to IV. Stage 0 is a mass that has not yet spread beyond the lobule or duct where it originated, which is referred to as carcinoma in situ. Stage IV is cancer that has spread to distant organs of the body.

Hormone status. Determining the hormone receptor status of breast cancer cells has become a common practice, as there are implications for treatment options and 
predictions of subsequent survival time based on the presence or absence of certain hormone receptors. The status of three receptor types is determined using tumor tissue samples, taken during diagnosis of breast cancer. These are estrogen receptor (ER), progesterone receptor (PR), and human epidermal growth factor receptor 2 (HER2). The most common combination is ER/PR positive (+) and Her2 negative (-) (68.9\%; Onitilo, Engel, Greenlee, \& Mukesh, 2009). The absence of all three hormone receptors is known as "triple negative" breast cancer. For an individual diagnosed with triple negative cancer, there are fewer treatment options, as receptor-targeted hormone therapy is not effective with these cases (Bauer, Brown, Cress, Parise, \& Caggiano, 2007). Triple negative status is associated with the shortest survival rate (Onitilo et al., 2009). The prevalence of various hormone receptor types varies across racial/ethnic groups. Triple negative breast cancer is highest among African American women (Bauer et al., 2007).

Treatment options. Multiple standard treatments are used to treat the different stages and types of breast cancer (Carlson et al., 2011). Localized disease is treated with surgery, radiation therapy, or both, whereas widely invasive or metastatic disease is treated with chemotherapy, endocrine therapy, biologic therapy, or combination therapy (Carlson et al., 2011). Surgery is performed in order to remove cancer from the breast. One type of surgery is breast-conserving, (e.g., lumpectomy or partial mastectomy), indicating that tumor is removed but the entire breast is not (Parajuli, 2015). Alternatively, patients may opt for total mastectomy, which is the surgical removal of the entire breast (Parajuli, 2015). Chemotherapy, the use of systemic drug treatment to either kill cancer cells or inhibit their division, may be used prior to surgery, a strategy known as neoadjuvant treatment (Parajuli, 2015). Chemotherapy is used to reduce the tumor size 
and therefore the amount of tissue to be removed at the time of surgery (Parajuli, 2015) Chemotherapy and/or radiation therapy, the use of radiation to kill or shrink cancer cells, may be used after surgical treatment (Jacobs \& Finlayson, 2010). Endocrine therapy is a treatment that blocks the effects of naturally occurring hormones that stimulate the growth of breast tissue (e.g., estrogen; Miller \& Ingle, 2002). Biologic therapy is drug treatment that activates the immune system to inhibit tumor growth and spread (Vachani \& Millar, 2015) and includes HER2-targeted therapy (Carlson et al., 2011).

Psychological symptoms. Breast cancer can be thought of as a series of stressors that relate to diagnosis, treatment, reduction in functional ability, changes in social and vocational roles, and concerns related to morbidity and mortality (Spiegel \& Sephton, 2001). Psychological distress can manifest as depressive symptoms, anxiety, insomnia, and increasing social isolation; all of which are common following a cancer diagnosis (Mertz et al., 2012). In a study of women with breast cancer, clinically significant levels of either anxiety or depression were observed in $34 \%$ of patients two months following diagnosis (Gallagher, Parle, \& Cairns, 2002). These symptoms decreased by six months post-diagnosis; however, rates of psychological illness remain higher among cancer patients in comparison with those of the general population (Gallagher et al., 2002). Additionally, women newly diagnosed with breast cancer experience greater role limitations related to emotional difficulties than those with stable disease (i.e., women who have completed adjuvant treatment and are at least six-month post-diagnosis, and women who have not experienced a recurrence; Hanson Frost et al., 2000).

Tumor progression. The spread of cancer from the primary site to lymph nodes and distant organs constitutes metastatic disease. Cancer cells and associated tissue 
develop "Hallmark" capabilities that promote tumor growth and spread (i.e., movement out of the site of origin into other tissues; Hanahan \& Weinberg, 2011). Many steps are essential for cancer to metastasize, including proliferation (i.e., growth or production of cancer cells; Guo \& Hay, 1999), angiogenesis (i.e., development of a blood supply; Lutgendorf, Sood, \& Antoni, 2010), detachment, and invasion (i.e., cancer cells must separate from their site and move through the body in order to invade other organs; Armaiz-Pena, Cole, Lutgendorf, \& Sood, 2013). Embolism, circulation, stopping in an organ and adhering to vessel walls, extravasation (i.e., the movement of cells from blood vessel to tissue; Miles, Pruitt, Golen, \& Cooper, 2007), and the establishment of a tumor microenvironment, and blood supply (Fidler, 2003; Hanahan \& Weinberg, 2011) are also steps needed for metastasis. Additionally, cancer cells must evade growth suppression, immune destruction, and apoptosis and stimulate inflammation that helps to promote the tumor (Hanahan \& Weinberg, 2011). Metastatic tumors are responsible for $90 \%$ of cancer deaths, as compared with primary tumors (Sporn, 1996).

\section{Stress and Health}

Psychological stress is defined as the appraisal of an event or circumstance as demanding or overwhelming to the point that coping resources will be taxed or exceeded (Lazarus \& Folkman, 1984). Neuroendocrine and immune stress responses that function in a normal manner (i.e., activated in response to threats to survival) are essential to leading a healthy life. Under acute stress conditions, these systems serve protective functions for the human body. However, when stress responses are frequently and repeatedly activated over an extended period (i.e., chronically activated), elevations in 
exposure to stress hormones occur and may have detrimental effects on other body systems causing a loss of flexibility of biologic responses to stress (McEwen, 1998).

When stress responses are chronically activated, a buildup of physiological damage can occur (McEwen, 2006), leading to stress-related diseases (Sapolsky, 2004). Specifically, chronic stress can affect biological processes (i.e., neuroendocrine and immune responses) that influence cancer progression (i.e., metastasis; Moreno-Smith, Lutgendorf, \& Sood, 2010). A biobehavioral model (Andersen, Kiecolt-Glaser, \& Glaser, 1994) indicates that cancer diagnosis and treatment can serve as a stressor sufficient to impact tumor growth and immune defenses through overactivation and dysregulation of neuro-endocrine-immune pathways, promoting the progression of cancer (Andersen et al., 1994).

\section{Stress Related to Ethnicity}

A person's ethnicity, and their identification with their ethnicity, can contribute to psychological stress. Racial or ethnic identification is defined as the "cognitive-affective representation of ethnic group membership within a person" (Contrada et al., 2001, p. 1779). Ethnicity-related stress can be defined as the appraisal of the environment with regard to one's ethnicity, which may lead to the anticipation of harm, either physical or psychological, or to the belief that harm has already occurred (Contrada et al., 2001).

A review article described three contributing factors to ethnicity-related stress: ethnic discrimination (i.e., unfair treatment of an individual based on their race/ethnicity), stereotype threat (i.e., the risk of conforming to a negative stereotype for the group that one belongs to), and own-group conformity pressure (i.e., being pressured to comply with expectations from the group that one identifies with; Contrada et al., 2000). Ethnicity- 
related stress may develop in response to a number of events, such as believing that there are threats to one's self-esteem, recognizing a lack of normalization by the group to which one belongs, and experiencing hopelessness related to one's ethnic identification (Clark, Anderson, Clark, \& Williams, 1999). Additionally, maintaining one's ethnic or cultural identity may contribute to stress due to pressure to conform to the group to which one belongs or to conform to the perceptions of norms and expectations of the in-group (Contrada et al., 2000). However, ethnic identity has also been shown to be positively related to quality of life (QOL) and overall well-being (Utsey, Chae, Brown, \& Kelly, 2002). Therefore, it is important to delineate what aspects related to ethnicity (e.g., racial discrimination) contribute to ethnicity-related stress and what facets may enhance wellbeing.

Certain life stressors are more often experienced in ethnic minority groups, which may promote stress in these individuals. These stressors are largely based on socioeconomic disadvantages (Lillie-Blanton \& Laveist, 1996; Harrell, 2000). As is well known, socioeconomic status (SES) covaries with ethnicity. Generally, SES can be characterized as a measure of opportunities, both educational and economic, that are available in an individual's environment (Lillie-Blanton \& Laveist, 1996). In a large longitudinal study that followed Baltimore schoolchildren for years, in adulthood the African American men earned less and had lower employment than the non-Hispanic $(\mathrm{NH})$ White men with equal educational attainment (Alexander, Entwisle, \& Olson, 2014). Additionally, African Americans were more likely to be single parents, and while African American women earned similar income to NH White women, because of the larger number of African American single-mother households, the family income was 
substantially lower compared to NH Whites (Alexander et al., 2014). Although all participants in the study were socioeconomically disadvantaged, it was determined that $\mathrm{NH}$ White participants had access to greater resources, especially with respect to obtaining employment, and these resources may be beneficial in creating a pathway toward better SES over time (Alexander et al., 2014). In a study of NH White and Hispanic lesbian, gay, and bisexual individuals an association between ethnicity and perceived stress was observed, and this relationship was mediated by SES, as well as lifetime discrimination (Kim \& Fredriksen-Goldsen, 2016). Furthermore, ethnic minorities experience greater perceptions of stress related to employment, financial strain, life discrimination, job discrimination, relationships, and early life (Sternthal, Slopen, \& Williams, 2011).

A biopsychosocial model of perceived racism can further illuminate the effects of ethnicity-related stress. Sociodemographic, psychological, and behavioral factors, as well as coping resources, interact in response to the perception of racial discrimination, playing a vital role in the impact of ethnicity-related stress on health outcomes (Clark et al., 1999). Both negative physical and psychological health outcomes have been observed in those who experience perceived racism (Clark et al., 1999) and ethnicity-related stressors (Contrada et al., 2000). Additionally, effects of both intergroup and intragroup racism may culminate in adverse health outcomes (Clark et al., 1999). Perceived racial discrimination has been shown to be associated with psychological distress (Sellers, Caldwell, Schmeelk-Cone, \& Zimmerman, 2003; Kessler, Mickelson, \& Williams, 1999), as well as depressed mood and anxiety (Kessler et al., 1999). The accumulation of ethnicity-related stress along with other general stressors may deem ethnic minorities at 
greater risk for mentally and physically detrimental effects than those who are within the ethnic majority, for example, NH Whites in the United States. In a sample of first year African American medical students, those with lower racial identification were less likely to endorse depressive and anxiety symptoms (Hardeman et al., 2016).

Disparities exist among ethnic minority groups in health status, morbidity, and mortality (Myers, 2009). It has been shown that ethnic differences in disease relate more to underlying social conditions (i.e., childhood poverty, inadequate education, low income, minimal employment, and separated living conditions), rather than to behavioral differences (e.g., coping style; Harrell, 2000). Socioeconomic factors also contribute substantially to ethnic health disparities (Lillie-Blanton \& Laveist, 1996). Also, ethnic health disparities may be due to one's surroundings (i.e., neighborhood and work conditions), social relations, and power arrangements (i.e., individual and community control and influence; Lillie-Blanton \& Laveist, 1996).

Ethnic minorities differ from the majority in the trajectory of chronic disease and in response to treatment (Myers, 2009). Ethnicity-related stress has been shown to accelerate the progression of a number of diseases, one of which is cancer (Myers, 2009). Specifically, adverse disease outcomes occur at higher rates in ethnic minorities, with African Americans experiencing the greatest burden (Myers, 2009). Breast cancer incidence is highest among NH Whites, but African Americans suffer the highest mortality (DeSantis et al., 2014). Additionally, in comparison with NH Whites, African American breast cancer patients are more likely to be diagnosed at an earlier age, and are diagnosed with more advanced stage disease or more aggressive cancer types (Middleton et al., 2003). 
Access to health care and receipt of treatment do play a role in the association between ethnicity and cancer. Ethnic minorities experience disparities in receipt of definitive primary (i.e., surgery and radiation) therapy, conservative surgery, and adjuvant treatments (i.e., chemotherapy and hormonal therapy; Shavers \& Brown, 2002). Additionally, NH Whites are more likely to receive aggressive cancer treatments (Shavers \& Brown, 2002). Evidence suggests there are relatively fewer ethnic differences in health care access in systems with universal accessibility (i.e., Department of Defense), supporting the notion that poor access may be a major determinant of ethnic differences in health outcomes (Mayberry, Mili, \& Ofili, 2000). Concerning cancer outcomes, the literature is less clear due to a lack of treatment data and limited quality of previous research (Mayberry et al., 2000). Therefore, further research should more broadly explore factors that contribute to the relationships observed between ethnicity and cancer to better understand ethnic differences in clinical characteristics (e.g., age and stage at diagnosis), as well as differences in treatment modalities and outcomes (e.g., morbidity and mortality).

Some data suggests that ethnic differences in health behaviors also explain a portion of the differences in cancer progression. A telephone survey of 42,044 households revealed that, in general, African Americans are more likely to smoke, report a lack of physical exercise, and be overweight compared to NH Whites (Shariff-Marco, Klassen, \& Bowie, 2010), all of which enhance the risk of breast cancer. Compared to NH Whites, Latino participants were more likely to binge drink and be overweight, Asian American participants were more likely to be less physically active, and American Indian/Alaskan Native participants were more likely to smoke, binge drink, report a lack 
of physical exercise, and be overweight (Shariff-Marco et al., 2010). These health behaviors may contribute to cancer progression (Calle, Rodriguez, Walker-Thurmond, \& Thun, 2003; Tammemagi, Neslund-Dudas, Simoff, \& Kvale, 2004; Vucenik \& Stains, 2012). The possible effect of health behavior differences on cancer outcomes is a nascent research area.

It is also possible that genetic differences related to ethnicity may explain some disease outcome variance. Indeed, genetic factors can interact with environmental ones to produce discrepancies in health outcomes (Caspi et al., 2003).Thus, gene-environment interactions related to alleles more prevalent in ethnic minorities may potentially explain some ethnic differences in cancer outcomes (Braun, 2002).

Research has primarily focused on perceived racism/racial discrimination as determinants of ethnicity-related stress. While this research aids in understanding interactions of ethnicity and stress, there are additional areas that warrant attention. Perceived stress in relation to minority ethnic identification should be investigated, as understanding these associations may help in identifying ethnic differences in the perception of stress. Additionally, exploring ethnicity-related stress and health outcomes may help to elucidate potential mechanisms that drive ethnicity-related health disparities.

\section{Neighborhood Stress}

Research has shown that neighborhood disadvantages may impact health more than individual socioeconomic and demographic factors (Ross \& Mirowsky, 2001). Neighborhood-level variables may include the built environment (e.g., the presence of toxic waste facilities, traffic exposure, and medical infrastructure; Matthews \& Yang, 2010). Additionally, neighborhood safety, residential stability, and measures of social 
disadvantage, including poverty, unemployment, and receipt of public assistance are considered social characteristics of neighborhoods (Matthews \& Yang, 2010).

Neighborhood factors can be viewed as a source of chronic stress that may have an adverse impact on health (Steptoe \& Feldman, 2001). Neighborhood factors that may influence the health of residents include the percentage of people that are poor, lack of education, are unemployed, and the percent of single mother households (Hill, Ross, \& Angel, 2005). In low socioeconomic neighborhoods, there are greater rates of cancer (Adler, Boyce, Chesney, Folkman, \& Syme, 1993). Furthermore, research has shown that even though the relationship between neighborhood-level stress and health of the residents has been established, the mechanisms of this relationship remain underexplored (Ellen, Mijanovich, \& Dillman, 2001; Ross \& Mirowsky, 2001).

Environmental stressors may "get under the skin" by way of psychological, physiological, and behavioral pathways. Indeed, unstable neighborhoods (i.e., high turnover and low homeownership), appear to negatively influence the health of the residents (Boardman, 2004). Given the importance of stress in the progression of cancer, it will be very informative to consider the role neighborhood factors in stress-cancer associations.

\section{Cancer-Related Stress}

Acute stress disorder (ASD) and post-traumatic stress disorder (PTSD) are classified as trauma- and stressor-related disorders in the fifth edition of the Diagnostic and Statistical Manual of Mental Disorders (DSM 5; American Psychiatric Association, 2013). ASD and PTSD are diagnosed after the development of a constellation of symptoms (i.e., intrusions, avoidance, negative alterations in mood or cognition, and 
changes in arousal or reactivity) following a traumatic event (American Psychiatric Association, 2013). In ASD the symptoms are present from three days to one month after the traumatic event, whereas PTSD is diagnosed when the symptoms endure longer than one month (American Psychiatric Association, 2013). It is important to note that DSM-IV allows for a life-threatening illness as a traumatic stressor; however, with changes to DSM-5, life-threatening illnesses are no longer considered a traumatic stress event (Swartzman, Booth, Munro, \& Sani, 2016). Therefore, it is possible that individuals with cancer may experience symptoms of PTSD (e.g., intrusions or avoidance) and no longer meet diagnostic criteria for PTSD.

Cancer-related stress differs from ASD and PTSD, as both are mental disorders recognized in DSM-5, whereas cancer-related stress is not a diagnosable mental disorder. Cancer-related stress includes two symptoms of PTSD, intrusions and avoidance. Intrusions include intrusive thoughts, nightmares, intrusive imagery, and dissociative-like re-experiencing, whereas avoidance includes numbing of responsiveness and avoiding feelings, situations, and ideas (Horowitz, Wilner, \& Alvarez, 1979). The presence of both symptoms indicates elevated cancer-related stress and more severe impact of illness (Mcbride, Clipp, Peterson, Lipkus, \& Demark-Wahnefried, 2000). Cancer-related ASD, cancer-related PTSD, and cancer-related stress have been observed throughout the breast cancer trajectory.

In a sample of 85 newly diagnosed (i.e., average of 11.65 days after diagnosis) breast cancer patients, those who experience high levels of intrusions or both intrusions and avoidance had the poorest psychological adjustment to diagnosis (Primo et al., 2000). In contrast, those who reported low levels of both symptoms had the lowest level of 
depressive and anxiety symptoms at six-month follow-up (Primo et al., 2000). In a study of 166 breast cancer patients, four met criteria for cancer-related ASD and two met criteria for cancer-related PTSD after diagnosis and prior to surgery (i.e., lumpectomy or mastectomy; Voigt et al., 2016). After chemotherapy (i.e., seven and one-half months after baseline assessment) and one year after baseline assessment, three of the patients in the study met criteria for either cancer-related ASD or cancer-related PTSD (Voigt et al., 2016). Of note, while not meeting criteria for ASD or PTSD, $82.5 \%$ of patients endorsed cancer-related PTSD symptoms at baseline, and 57.3\% endorsed symptoms at one-year follow-up (Voigt et al., 2016). In a sample of 437 women with breast cancer, three percent $(\mathrm{n}=13)$ met criteria for PTSD, diagnosed with a Structured Clinical Interview for DSM-IV, at baseline (i.e., average of 65.4 days after diagnosis; Kwakkenbos, Coyne, \& Thombs, 2014). At three month follow-up, nine patients met criteria for PTSD (Kwakkenbos et al., 2014). One-hundred seventeen patients with primary breast cancer within six months of a diagnosis experienced cancer-related stress at a level that is moderately high (Koopman et al., 2002). In a sample of 150 women with breast cancer, high levels of intrusions and avoidance following diagnosis and prior to surgery remained two years later (Arnaboldi et al., 2014).

Women with metastatic breast cancer also report high rates of intrusions and avoidance, rates that are comparable to those who have had experienced other traumatic stressors such as survivors of multiple shootings and motor vehicle accidents (Butler, Koopman, Classen, \& Spiegel, 1999). However, it appears that levels of cancer-related stress are lower among women with primary breast cancer (Koopman et al., 2002a) as compared with metastatic patients (Butler et al., 1999). In a sample of women who had 
completed treatments for breast cancer, five to ten percent met criteria for the diagnosis of cancer-related PTSD (Cordova et al., 1995). A recent meta-analysis revealed that survivors of cancer are 1.66 times more likely to have PTSD than controls without cancer (Swartzman et al., 2016).

Possible factors that may increase the likelihood of cancer-related PTSD include younger age at diagnosis, receiving chemotherapy, and time since diagnosis (i.e., PTSD rates appear to decrease over time; Swartzman et al., 2016). Cancer-related stress has been shown not to be associated with time since diagnosis (Mcbride et al., 2000). It appears that cancer-related stress is present from the time of a breast cancer diagnosis and remains throughout the treatment trajectory. Therefore, the stress associated with a breast cancer diagnosis may be psychologically impactful and salient in the long-term for the patient, rather than only present initially following the diagnosis (Mcbride et al., 2000).

\section{Circadian Rhythms}

Healthy circadian regulation. Human physiology is synchronized with the rotation of the earth by the hypothalamic suprachiasmatic nuclei (SCN). The central circadian clock, located in the SCN, synchronizes the peripheral clocks of most mammalian cells through an array of circadian physiological rhythms that include rest/activity rhythms, hormonal secretions, body temperature, and feeding (Mohawk, Green, \& Takahashi, 2012; Schibler et al., 2015). Light-dark cycles are the strongest zeitgebers of central control over peripheral rhythms (Schibler et al., 2015). The traditional view of the relationship between the $\mathrm{SCN}$ and the peripheral cellular clocks is believed to be hierarchal in nature, with the central clock of the SCN regulating the timing of peripheral clocks; however, recent research is beginning to show that the 
relationship may be more "federated," such that various oscillators are equal to one another (Husse, Eichele, \& Oster, 2015, p. 1120). Therefore, it is likely that peripheral clocks are influenced by independent zeitgebers (e.g., light-dark cycles, hormones, bloodborne signals, body temperature; Husse et al., 2015).

Circadian rhythms and cancer. Research has shown that dysregulated circadian rhythms, specifically shift work that involves the disruption of circadian rhythms, is probably carcinogenic to humans, which makes it a Class 2A carcinogen (Straif et al., 2007). Shift work is associated with changes in rest/activity rhythms, such that patterns of activity become deregulated and circadian synchronization is lost, which can lead to dysregulation of the endocrine and immune system (Herichova, 2013). Research regarding the prognostic value of circadian disruption and cancer risk has confirmed higher incidence of breast cancer in nurses who worked night shift for many years (Davis, Mirick, \& Stevens, 2001; Schernhammer et al., 2001; Schernhammer, Kroenke, Laden, \& Hankinson, 2006).

Changes to circadian rhythms occur in tumor tissues, animal models with tumors, and human cancer patients. As a tumor progresses, circadian alterations worsen (Mormont \& Levi, 1997). Research on effects of lesions to the SCN in murine models has revealed changes to implanted tumors that result in accelerated growth and shorter survival (Filipski et al., 2002; Filipski, Li, \& Levi, 2006). When a tumor is present, it is possible that disruption of circadian rhythms may be a response to biological effects of the tumor, or may be the consequence of the physiological and psychological demands of disease (Eismann, Lush, \& Sephton, 2010).

\section{Circadian disruption.}


Rest/activity rhythms. Rest/activity rhythms, patterns of activity levels over multiple days, are strong indicators of central clock activity and have been shown to be related to cancer outcomes (e.g., QOL and survival; Mormont \& Waterhouse, 2002). Circadian rhythms can be measured noninvasively in humans through the use of wristworn accelerometers, known as actigraphs. While polysomnography is widely considered the "gold standard" in measuring sleep and related variables, actigraphy is a valid and reliable approach to measuring rest/activity rhythms (Ancoli-Israel et al., 2003). Given its portability, this method is often preferred in research.

Rest/activity rhythms have been shown to be disturbed prior to and during chemotherapy treatment in women with breast cancer (Ancoli-Israel et al., 2006; Savard et al., 2009). Additionally, women with metastatic breast cancer have dysregulated rest/activity rhythms (Koopman et al., 2002). While relationships have been observed between rest/activity rhythms, QOL, and cancer survival in colorectal samples (Innominato et al., 2009; Lévi et al., 2014; Mormont et al., 2000; Mormont \& Waterhouse, 2002), limited research has been conducted in breast cancer samples. In a sample of 57 presurgical breast cancer patients, no relationship was observed between rest/activity rhythms and QOL (Rebholz et al., 2016). Continued research exploring the relationship between rest/activity rhythms and cancer outcomes (i.e., QOL and survival) within breast cancer patients may be beneficial in understanding relationships between rest/activity rhythms and cancer outcomes and mechanisms that may contribute to the associations. Additionally, research with this focus may help in identifying whether changes occur throughout the cancer trajectory (e.g., presurgical, between surgery and adjuvant treatment, during adjuvant treatment, and after the completion of treatment). 
Hypothalamic pituitary adrenal (HPA) axis. The HPA axis is one of the two major physiological stress responses systems. It is likely a zeitgeber by which the SCN coordinates peripheral oscillators, and loss of its circadian rhythmicity is prognostic for mortality among several cancer types (Innominato et al., 2009; Lévi et al., 2014; Mormont, Bogdan, Cormont, Touitou, Levi, 2002; Sephton, Sapolsky, Kraemer, \& Spiegel, 2000; Sephton et al., 2013). The HPA axis is activated by perceived stress (McEwen, 1998; Miller, Chen, \& Zhou, 2007). Specifically, to maintain physiological stability when presented with a challenge, whether real or perceived, the HPA axis is activated. Frequent and repeated (i.e., chronic) activation of the HPA axis can lead to the development and progression of disease, including cancer, heart disease, obesity, and diabetes (McEwen \& Stellar, 1993; Smith \& Vale, 2006).

The primary glucocorticoid produced by the human body is cortisol, which rises in the blood within a few minutes of experiencing a stressor (Jameson, 2003), and peaks about 20 minutes after the onset of a continued stressor. The cortisol awakening response (CAR) is a measure of HPA axis activity following awakening (Kudielka \& Wüst, 2010). A positive relationship between response to awakening and perception of chronic stress has been observed among 100 individuals (Schulz, Kirschbaum, Prüßner, \& Hellhammer, 1998), among 212 individuals chronically stressed from work overload (Schlotz, 2004), and among 66 public school teachers (Pruessner, Hellhammer, \& Kirschbaum, 1999). CAR is only weakly associated with the response of the HPA axis to acute stress (Kudielka \& Wüst, 2010). Cortisol levels are generally low during the late evening hours, increase rapidly in the morning prior to waking, and peak just after waking. Levels then gradually decline throughout the day (Chan \& Debono, 2010). Examining the rhythmic 
pattern of cortisol levels throughout the day yields the diurnal cortisol slope, which reflects the rate of change of cortisol secretion. Unhealthy slopes are those that appear consistently flat with little variance, regardless of the whether there is a high or low intercept for the slope. In cancer patients, cortisol slopes often appear to be idiosyncratic with erratic peaks and troughs, as well as phase changes (Touitou et al., 1995). Although the HPA axis is under circadian control, it can also be influenced by stress and other factors.

\section{Immune Function}

Healthy immune defenses. The immune system is the human body's natural defense against illness and disease, which includes a complex array of cellular responses that are coordinated in order to protect the body. Cell-mediated inflammatory processes are brought about by $\mathrm{T}_{\mathrm{H}^{-1}}$ cells (Mosmann $\&$ Sad, 1996). $\mathrm{T}_{\mathrm{H}^{-1}}$ cytokines may initiate both cytotoxic and inflammatory functions, while antibody production is linked to $\mathrm{T}_{\mathrm{H}}-2$ cytokines (Mosmann \& Sad, 1996). Type 1 cytokines are classified as pro-inflammatory and type 2 as anti-inflammatory (Elenkov \& Chrousos, 1999).

Immune function and cancer. Psychoneuroimmune pathways may mediate observed relationships between stress and cancer outcomes (Andersen et al., 1994; Antoni et al., 2006; Eismann et al., 2010; Lutgendorf et al., 2010). The first evidence of a link between inflammation and cancer came in the 1800 s when the presence of leukocytes was discovered in tumors (Grivennikov, Greten, \& Karin, 2010). Immune cells may impact cancer cells by producing cytokines, chemokines, growth factors, prostaglandins, and reactive oxygen and nitrogen species (Grivennikov et al., 2010). 
Several specific immune parameters have been implicated in the relationship between immune function and tumor progression. Vascular endothelial growth factor (VEGF) is released by cells that are not receiving sufficient oxygen. It promotes angiogenesis - the growth of new blood vessels - and is released by some tumors. In ovarian cancer cells, stress-related hormones (i.e., epinephrine and norepinephrine) have been shown to increase the production of VEGF (Lutgendorf et al., 2003). VEGF overproduction is linked to both growth and metastasis of solid tumors (Veikkola \& Alitalo, 1999). Matrix metalloproteinase (MMP)-9 helps to facilitate tumor invasion and metastasis, particularly in response to signals from stress hormones (Sood et al., 2006). In the early stages of cancer, transforming growth factor (TGF) $-\beta$ suppresses development; however, the invasiveness and potential of metastasis are enhanced by TGF- $\beta$ in the later stages (Zheng, Safina, \& Bakin, 2008). Large quantities of TGF- $\beta$ are produced in the tumor microenvironment, and it is generally immunosuppressive; however, the exact means by which this cytokine interacts with the tumor is dependent upon the tumor microenvironment and the other immune parameters that are present (Zamarron \& Chen, 2011). Additionally, TGF- $\beta$ serves to inhibit macrophage activation, which may decrease production of proinflammatory immune parameters (Zamarron \& Chen, 2011). Downstream effectors of tumor growth are triggered by interleukin-6 receptor (IL-6R; Grivennikov \& Karin, 2008). Monocyte chemoattractant protein (MCP)-1 also contributes to inflammation and the progression of tumors (Fujimoto et al., 2009). Myeloid-derived suppressive cells and the cytokines that are secreted, including interleukin (IL)-6, tumor necrosis factor (TNF)- $\alpha$, and IL-1 $\beta$, are vital in the development of tumors (Zamarron \& Chen, 2011). Additionally, immune parameters that are 
proinflammatory in nature, including IL- $1 \beta$, TNF- $\alpha$, IL-6, IL-12, and interferon (IFN)- $\gamma$, promote tumors by stimulating proliferation, angiogenesis, and metastasis (Mantovani, Allavena, Sica, \& Balkwill, 2008).

\section{Theoretical Models}

It has been proposed that psychoenuroendocrine pathways, including activation of the HPA axis, may directly or indirectly influence cancer progression (Eismann et al., 2010). It is suggested that a relationship between the HPA axis and psychosocial factors exist and that this relationship may influence cancer progression (Eismann et al., 2010). Stressors, and an individual's psychological response to stressors, can trigger neuroendocrine activity (Antoni et al., 2006). In turn, endocrine stress responses can impact the tumor microenvironment and promote migration and/or invasion of cancer cells, as well as stimulate angiogenesis, and alter immune disease-resistance capability (Antoni et al., 2006).

In a related theoretical model, it has been proposed that biobehavioral factors influence cancer-related processes through biological, neuroendocrine, and immune factors (Antoni et al., 2006). Such biobehavioral factors may include life stress, psychological processes, and health behaviors (Antoni et al., 2006). A similar model explores the relationships between psychosocial processes, biological factors, health behaviors, life stress, and psychological interventions and posits neuroendocrine and immune pathways leading to cancer progression and mortality (Lutgendorf \& Costanzo, 2003).

These models illustrate the complexity of factors relevant to the progression of cancer. While cancer-related stress has been explored in the context of some of these 
models, no studies have examined whether stress related to ethnicity, neighborhood factors, and cancer-related stress may impact cancer progression via the hypothesized pathways. Therefore, exploring the relationships of psychosocial stress and physiological disruption with each other and in relation to cancer progression-free survival contributes to a missing area of the literature. By better understanding these relationships within the cancer context, there is potential to begin to elucidate factors that contribute to cancer disparities and identify interventions that may improve quantity and/or quality of life in patients with cancer.

\section{Proposed Model}

The proposed model (Figure 1) builds upon our lab's prior of model of circadian effects on tumor progression (Eismann et al., 2010). This study proposes interrelationships of psychosocial stress related to ethnicity, neighborhood stressors, and the cancer diagnosis and suggests specific associations between psychosocial stress, physiological disruption, and medical outcomes of cancer (progression-free survival time). A significant contribution of this model is the focus on multiple domains of psychosocial and physiological distress. The measures of psychosocial stress take into account an individual's ethnicity; their environment, by exploring stress associated with their neighborhood; and their individual response to a cancer diagnosis. Additionally, the measures of physiological disruption take into account circadian rhythms measured by rest/activity rhythms and endocrine hormone secretion, as well as functioning of the immune system. By exploring multiple domains of stress, one can begin to investigate what areas are most salient in cancer patients that have not yet received treatment. Given that treatment for cancer affects biological parameters, studying presurgical patients who 
have yet to begin treatment allows for exploration of associations while the tumor is still present and prior to biological changes brought about by treatment. This can help us to understand the relationship between stress and physiological disruption, which can provide strong implications for medical and psychological clinical interventions.

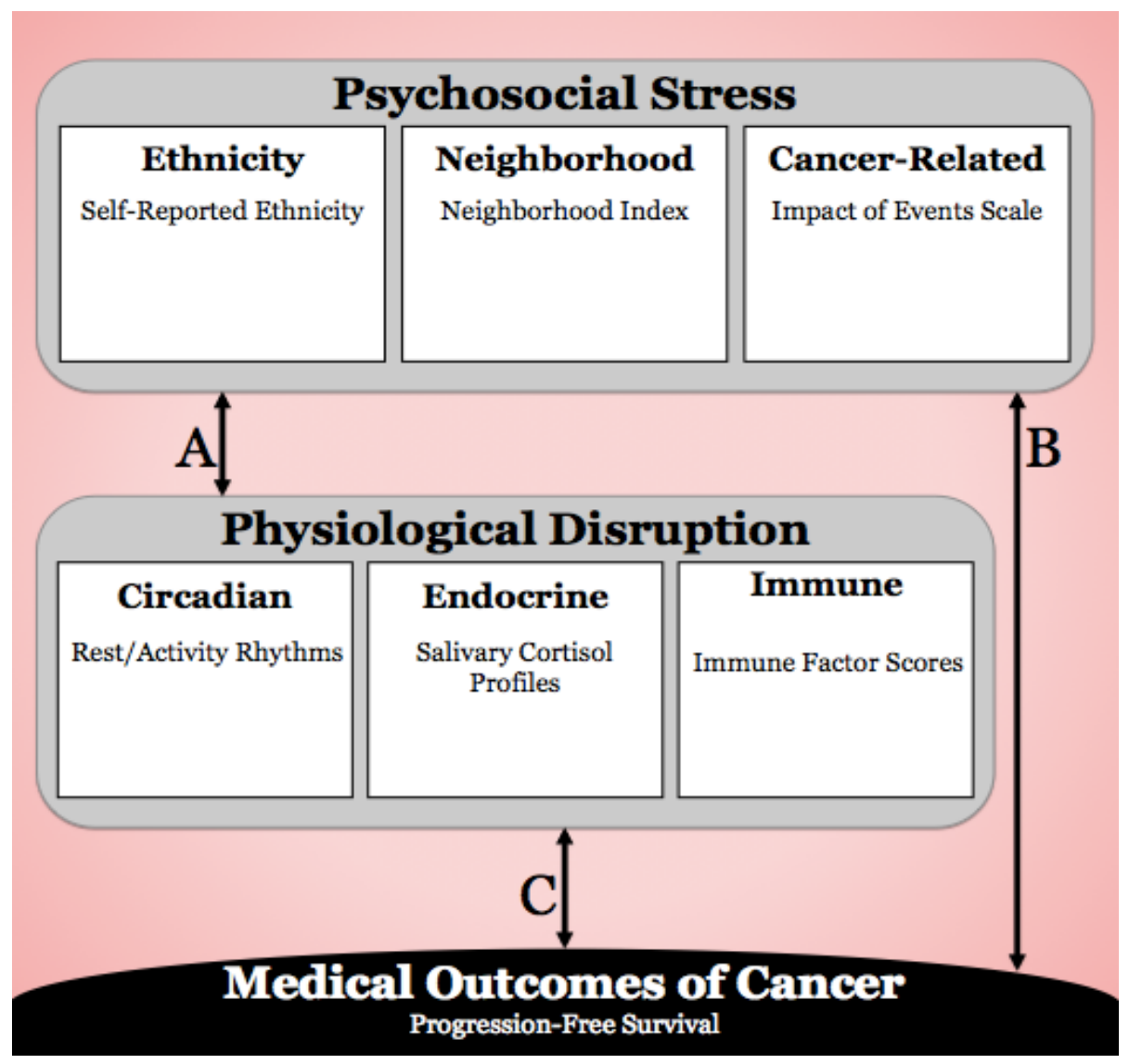

Figure 1. Study aims.

\section{Ethnicity and Physiological Disruption (Figure 1 Arrow A)}

\section{Associations with circadian disruption.}

Rest/activity rhythms. No research could be found exploring ethnic differences in rest/activity rhythms. Research has focused on sleep-related ethnic differences, which is likely correlated with rest/activity rhythms. Research has shown no differences between ethnicities in sleep architecture (Rao et al., 1999). Other studies have demonstrated that 
African Americans had longer rapid eye movement sleep, greater total sleep time, a lower percentage of deep sleep (Profant, Ancoli-Israel, \& Dimsdale, 2002), and less slow wave sleep (Stepnowsky, Moore, \& Dimsdale, 2003). Among older adults, sleep disturbances were associated with ethnicity, specifically with European Americans having worse sleep disturbance (Jean-Louis et al., 2001). This is in contrast to research showing African American older adults having more sleep difficulties, including daytime sleepiness and problems falling asleep (Ancoli-Israel et al., 1995), difficulties initiating and maintaining sleep, as well as early morning awakening (Jean-Louis, Kripke, Ancoli-Israel, Klauber, \& Sepulveda, 2000), short or long sleep duration (Hale \& Do, 2007), greater variation in day-to-day sleep duration, lower sleep efficiency, greater wake after sleep onset, lower total sleep time (Lemola et al., 2013), and greater odds of daytime sleepiness (Chen et al., 2015).

Mixed results have been observed with regard to ethnic differences in sleep. Research has shown ethnic differences in sleep architecture and sleep, while other research has not observed these differences. It is possible that the differences in results are due to differences in methodology and study populations. Therefore, much research remains in order to better elucidate the relationships between ethnicity and rest/activity rhythms and potential factors that help to explain these relationships. Factors could include psychosocial variables, such as racial/ethnic discrimination, or genetic factors. It is also possible that rest/activity rhythms help to explain ethnic health disparities.

Hypothalamic pituitary adrenal (HPA) axis. Ethnic differences have been observed in cortisol, such that, after controlling for both education and managerial status, greater overall salivary cortisol secretion was observed for European Americans than 
African Americans (Bennett, Merritt, \& Wolin, 2004). An interaction of ethnicity and education was observed, with lower educated African American participants having lower levels of cortisol upon awakening (Bennett et al., 2004). It is suggested that low waking cortisol could be an indication of chronic stress which, in turn, may influence systemic HPA axis dysregulation (Bennett et al., 2004).

Lower waking salivary cortisol and higher levels at bedtime were observed in African Americans compared to White young adults, which is indicative of a flattened cortisol slope (Cohen et al., 2006). Similarly, among adolescents, both African Americans and Hispanics had flatter salivary cortisol slopes than Caucasians (DeSantis et al., 2007). Flattened slopes were observed in African American and Hispanic middle to older age participants, indicating the possibility that a below normal salivary cortisol response occurs due to chronic stress (Hajat et al., 2010). Additionally, among middle to older adults, African Americans had lower waking salivary cortisol and a slower decline in the afternoon (Samuel, Roth, Schwartz, Thorpe, \& Glass, 2016), which is indicative of a less healthy diurnal cortisol pattern. However, no ethnic differences have been observed in urinary cortisol levels among middle-aged adults (Masi, Rickett, Hawkley, \& Cacioppo, 2004).

Among women who were pregnant, African Americans had lower levels of plasma cortisol, specifically with a decrease in levels from 24-26 weeks gestation to 3032 weeks gestation (Glynn, Schetter, Chicz-DeMet, Hobel, \& Sandman, 2007). Additionally, an association between cumulative stress and flat salivary cortisol rhythms was seen in African American women who were pregnant; however, this association was not observed in Hispanic women (Suglia et al., 2010). A possible explanation for this 
association could be due to the African American women having higher levels of stress, or Hispanic women having greater social support coping strategies (Suglia et al., 2010). Furthermore, salivary cortisol levels have been explored among those whom are caregivers of individuals with dementia. Ethnicity was associated with cortisol slope with African American caregivers having flatter slopes (McCallum, Sorocco, \& Fritsch, 2006) and in a sample of caregivers and non-caregivers, Hispanic women had flatter diurnal cortisol slopes than NH Whites (Gallagher-Thompson et al., 2006).

Cortisol, when measured in saliva, has been shown to differ in NH Whites and ethnic minorities, with less cortisol secretion and flattened slopes observed in ethnic minorities. Differences in plasma cortisol levels have also been observed. However, in a study exploring urinary cortisol, no ethnic differences were observed. This lack of a difference in only urinary cortisol, and neither salivary nor plasma cortisol could be due to the difference in the collection of bodily fluids in which cortisol was measured.

Associations with immune function. Ethnic differences with regard to immune function have been explored to a limited degree. Genetic differences for cytokines have been observed, such that African Americans have significant differences compared to $\mathrm{NH}$ Whites in the distribution of polymorphic alleles for some cytokines; therefore, inheriting certain cytokine genes may be determined in part by ethnicity and have implications for the optimization of immunosuppressive regimes in those receiving transplants (Hoffmann et al., 2002) and those with Hepatitis C (Zein, Germer, El-Zayadi, \& Vidigal, 2004). Genotypes that upregulate inflammation are more common in African American women than Whites (Ness, Haggerty, Harger, \& Ferrell, 2004). Additionally, among newborns, African Americans have higher frequencies of some alleles that have been implicated in 
cancer risk; which may help to explain disparities in the incidence of certain cancers (Zabaleta et al., 2008).

In addition to cytokine allelic distribution differences, limited research has explored ethnic differences in circulating immune parameters. IL-8 and granulocyte colony-stimulating factor (G-CSF) levels were higher and neutrophil count was lower in African participants compared to Caucasians, (Mayr et al., 2007) and greater levels of IL6 and C-reactive protein (CRP) have been observed in African Americans compared to NH Whites (Stowe, Peek, Cutchin, \& Goodwin, 2010). However, NH Whites have been shown to have higher levels of IL-4, IL-5, IL-8, TNF- $\alpha$, and IFN- $\gamma$ compared to African Americans (Pine et al., 2016). Furthermore, elevated levels of IL-1 $\beta$, IL-10, TNF- $\alpha$ were associated with lung cancer among African Americans and elevated levels of IL-6 and IL-8 were associated with lung cancer in both NH Whites and African Americans (Pine et al., 2016).

There is a dearth of literature regarding ethnic differences in immune function, particularly circulating immune parameters. However, given the limited research, this appears to be an area that may help to explain ethnic disparities in the development and progression of disease. Additional research with regard to immune function and the relationships between ethnicity and disease may help to illuminate mechanisms by which ethnic disparities are observed, particularly in patients with cancer.

\section{Neighborhood Stress and Physiological Disruption (Figure 1 Arrow A)}

\section{Associations with circadian disruption.}

Rest/activity rhythms. Research regarding neighborhood stress and rest/activity rhythms has not been conducted; however, is an important area to study given the 
relationships observed between chronic stress and disruption of sleep. In neighborhoods characterized as high stress due to crime, there are more self-reported sleep difficulties among its residents (Steptoe, O’Donnell, Marmot, \& Wardle, 2008). Residents in neighborhoods with high levels of stress based on safety, cleanliness, and noise report worse sleep quality (Hill, Burdette, \& Hale, 2009). Furthermore, sleep quality has been shown to moderate the relationship between neighborhood stress and psychological distress (Hill et al., 2009) and mediate the relationship between neighborhood stress and physical health (Hale, Hill, \& Burdette, 2010), as well as the relationship between neighborhood stress and psychological distress (Hill et al., 2009).

The limited research has shown relationships between variables of neighborhood stress and self-reported sleep. Research regarding neighborhood stress and rest/activity rhythms may be beneficial in further understanding possible relationships. It is likely that a variety of factors related to neighborhood stress, perceived and observed, may be related to rest/activity rhythms, in addition to sleep. Further, there may be different types of neighborhood stress (e.g., socioeconomic or violence) that may be more predictive of rest/activity rhythms than others.

Hypothalamic pituitary adrenal (HPA) axis. It is possible that neighborhood stress can influence disease through neuroendocrine pathways (Steptoe \& Feldman, 2001). Flattened salivary cortisol rhythms have been observed in individuals who live in neighborhoods with high levels of both perceived (e.g., perceived violence) and observed (e.g., homicide rates) stressors (Karb, Elliott, Dowd, \& Morenoff, 2012). Flattened diurnal slopes and flatter decline from 30 minutes post-awakening to about two hours post-awakening were associated with neighborhood poverty (Hajat et al., 2015). African 
Americans with high glucocorticoid genetic sensitivity living in neighborhoods with low SES had lower waking cortisol (Coulon, Wilson, Horn, Hand, \& Kresovich, 2015). Neighborhood violence was associated with low waking cortisol; however, neighborhood poverty associations with salivary cortisol in this sample were weaker and less consistent (Do et al., 2011). Additionally, African American children with greater exposure to neighborhood stress had higher levels of serum cortisol; however, this association was not observed in European American children (Dulin-Keita, Casazza, Fernandez, Goran, \& Gower, 2012).

Previous research has shown relationships between neighborhood stress and endocrine disruption. These associations have been seen for both perceived and observed stressors. Given the relationships between chronic stress and dysregulation of the HPA axis, and knowing that neighborhood stress is chronic in nature, exploring the associations of neighborhood stress and dysregulation of the HPA axis is imperative in understanding potential mechanisms by which neighborhood stress impacts health (Ross \& Mirowsky, 2001). Further research could help to elucidate the relationships between neighborhood stress and cortisol, which could have strong implications for progression of disease, including cancer.

Associations with immune function. Limited research has been conducted with regard to neighborhood stress and immune function. In a study of African American women, an association was seen between neighborhood SES and CRP, such that those living in the poorest neighborhoods had worse biomarker levels compared to those living in neighborhoods with better SES (Cozier et al., 2016). Additionally, elevated levels of CRP were observed in children living in areas with high poverty or crime (Broyles et al., 
2012). The relationship between elevated CRP and low neighborhood SES was also detected in a sample of Mexican-American women (Gallo et al., 2012).

It has been suggested that immune function may be a mediating factor in the relationship between neighborhood stress and disease (Steptoe \& Feldman, 2001). By focusing on the possibility of an association between neighborhood stress and immune function, it may be possible to begin to better understand the role of these variables in various disease processes, including cancer. It appears that CRP, a protein component that elevates in concentration in the acute phase of inflammation (Koj, 1974), and possibly other pro-inflammatory cytokines may be immune markers of interest in relation to neighborhood stress.

\section{Cancer-Related Stress and Physiological Disruption (Figure 1 Arrow A) Associations with circadian disruption.}

Rest/activity rhythms. Daily intrusions have been shown to be associated with consistency of rest/activity rhythms over several days, as well as sedentary behaviors during the day (Dedert et al., 2012). Additionally, daily avoidant coping was also associated with consistency of rest/activity rhythms over several days, sedentary behaviors during the day, and restlessness at night (Dedert et al., 2012). Differences in sleep have also been observed in a sample of presurgical breast cancer patients, the night prior to surgery intrusive thoughts were associated with less sleep duration and lower sleep efficiency (Wright, Schnur, Montgomery, \& Bovbjerg, 2010). In breast cancer survivors, intrusive thoughts were shown to be related to self-reported symptoms of insomnia (Taylor et al., 2012). 
Within samples of women with breast cancer and breast cancer survivors, research has shown relationships between components of cancer-related stress (i.e., intrusions and avoidance) and rest/activity rhythms, sleep, and symptoms of insomnia. More research has focused on associations with intrusions, rather than avoidance. This is expected given the established relationship between intrusive thoughts and sleep disturbance (Harvey, 2002; Harvey, Tang, \& Browning, 2005; Wicklow \& Espie, 2000). While focusing on intrusions seems intuitive, avoidance may also be associated with rest/activity rhythms, particularly daytime sedentariness.

Hypothalamic pituitary adrenal (HPA) axis. Cancer-related stress and its associations with endocrine activation have also been explored. Relationships between intrusions and alterations in endocrine activation have been observed in individuals who have suffered a psychological trauma (Yehuda, 2002). Among healthy women, breast cancer-specific intrusions were related to greater levels of cortisol during a work period, which was considered a life stressor in this study (Dettenborn, James, Valdimarsdottir, Montgomery, \& Bovbjerg, 2006). In this same sample of women, daily intrusions and daily avoidant coping was not related with measures of salivary cortisol (Dedert et al., 2012). Exploring the relationship between cancer-related stress and endocrine disruption allows for a better understanding of the role a cancer diagnosis and the associated symptoms of stress plays in changes to cortisol. In turn, this may be beneficial in elucidating effects on chronic disease.

Associations with immune function. Generally, the immune system is adaptive and responds to various signals from the body, including distress. How the immune system reacts is dependent upon whether the stressor is acute or chronic in nature. Acute 
stressors, which are time-limited, upregulate natural immunity (e.g., inflammation) and suppress specific immunity (e.g., proliferation), whereas chronic stressors trigger global immunosuppression (Segerstrom, 2004). Specifically, psychosocial stressors and depression are associated with decreased cytotoxic T-cell and natural killer cell activities, which influence immune processes of tumors (Reiche, Morimoto, \& Nunes, 2005;

Reiche, Nunes, \& Morimoto, 2004). Among breast cancer patients that have completed surgery, cancer-related stress has been shown to be related to inhibition of cellular immune response (Andersen et al., 1998), declines in natural kill cell activity and IFN- $\gamma$ (Von Ah, Kang, \& Carpenter, 2007), and elevations of IL-1R $\alpha$ (Mundy-Bosse, Thornton, Yang, Andersen, \& Carson, 2011). More research regarding the effects of cancer-related stress, including intrusions and avoidance, and their influence on immune function may be advantageous in understanding mechanisms of cancer progression.

\section{Psychosocial Stress and Medical Outcomes of Cancer (Figure 1 Arrow B)}

Associations with ethnicity. Much disparity exists between ethnic groups with regard to the progression of cancer, which can encompass advanced stage disease, metastasis, recurrence, and mortality. Data from the National Center for Health Statistics has been compiled regarding cancer mortality rates, as well as projections for the number of cancer deaths for the year 2014 (Siegel et al., 2014). This epidemiological data indicates five-year survival rates are lower among African Americans than for Whites for female breast, colorectum, esophageal, kidney and renal pelvis, liver and intrahepatic bile duct, lung and bronchus, melanoma of the skin, non-Hodgkin lymphoma, oral cavity and pharynx, ovary, prostate, urinary bladder, uterine cervix, and uterine corpus cancer 
(Siegel et al., 2014). The only cancer site with equal five-year survival between ethnic groups is pancreas (Siegel et al., 2014).

By observing death rates in this epidemiological study, ethnic disparities are evident. The death rates for cancer are 207.7 in 10,000 among African Americans and 172.4 in 10,000 among Whites (DeSantis et al., 2007). It is suggested that barriers to care, including socioeconomic disparities, may help to explain the ethnic differences observed in cancer outcomes; however, research has suggested that these differences remain after controlling for such factors (DeSantis et al., 2007).

The question of ethnicity-related differences in cancer outcomes is one that is complex in nature, specifically in that associations with minority status are not all in the same direction. Additionally, differences in cancer outcomes may exist within our general conceptualization of ethnic groups. For example, differences in Chinese Americans, Japanese Americans, and Korean Americans may be observed; however, we would conceptualize all of the three ethnic subgroups as Asian Americans. Research has explored the differences in cancer characteristics and mortality among ethnic groups for various cancer tumor sites.

It has been shown that African American women have a significantly greater risk of more advanced breast cancer, as measured by larger tumors (Haas et al., 2008), more advanced cancer stage at diagnosis (Bradley, Given, \& Roberts, 2001; Haas et al., 2008; Komenaka et al., 2010; Li, Malone, \& Daling, 2003; Ooi, Martinez, \& Li, 2011), higher grade tumors (Chlebowski et al., 2005; Li et al., 2003), a higher likelihood of negative hormone receptor status (Chlebowski et al., 2005; Komenaka et al., 2010), and greater distant recurrences (Komenaka et al., 2010), compared to NH White women. However, 
one study found no differences in tumor size or stage among these ethnic groups (Chlebowski et al., 2005). Furthermore, African Americans with breast cancer have been shown to have greater disease-specific mortality (Bradley et al., 2001; Chlebowski et al., 2005; Du, Lin, Johnson, \& Altekruse, 2011; Haas et al., 2008; Komenaka et al., 2010; Li et al., 2003; Ooi et al., 2011; Perkins, Cooksley, \& Cox, 1996) and overall mortality (Du et al., 2011; Komenaka et al., 2010), compared to NH White women.

The differences in disease-specific mortality remained after controlling for either stage or Medicaid/Medicare status (Bradley et al., 2001), body mass index, age, tumor stage, and whether the patient was in the observational or clinical arm of the study (Chlebowski et al., 2005), receipt of adequate care (Haas et al., 2008), age and employment (Komenaka et al., 2010), and stage, SES, and treatment (Perkins et al., 1996). The differences in overall survival remained after controlling for patient characteristics, tumor characteristics, income, poverty, education, treatment, and health insurance (Du et al., 2011), as well as after controlling for clinical stage and age at diagnosis (Komenaka et al., 2010). However, after controlling for age at diagnosis, clinical stage, and hormone status, disease-specific survival was no longer significantly different between ethnic groups and when controlling for estrogen-receptor status, overall survival was not significantly different (Komenaka et al., 2010).

The majority of research regarding ethnic differences in cancer outcomes has focused on differences in African American and NH White women with breast cancer. Little research has explored ethnic differences between other cancer types or in other ethnicities. This is an area that much improvement is needed. Epidemiological studies are the primary method of exploring ethnic differences in cancer outcomes. Obtaining data 
from cancer registries and surveillance programs may not be very informative, as it is possible that considerable data is missing and the data that are available often are dichotomous, leaving little room to conduct complex analyses. For example, Surveillance, Epidemiology, and End Results Program (SEER) public use data often does not contain detailed information on tumor characteristics, cancer treatment, and prognosis (Yu et al., 2009). Data from large databases are excellent for helping to recognize areas and trends in cancer outcomes. However, the results from such datasets should inform cross-sectional, experimental, and longitudinal research to understand and explain ethnic cancer disparities.

Access to care does not seam to explain much of the variance in cancer outcomes. It appears that presenting at a later stage to physicians, which is a strong prognostic indicator of mortality, does not explain why African Americans have poorer outcomes. Therefore, it is not an issue of incidence, but rather other factors are playing a role in the differences in cancer outcomes that are observed.

A theoretical model has been introduced to help elucidate racial disparities in cancer morbidity and mortality. Several factors have been proposed to explain differences in cancer mortality between ethnic groups. Patient factors, provider factors, and the health system are embedded in social, economic, and the public health landscape in a proposed model of mortality rate ratio among individuals with cancer (Blackman \& Masi, 2006). Patient factors include many important features of the disparities seen in mortality, including, among others, cultural attitudes and beliefs, being Non-English speaking, and a delay in seeking medical care (Blackman \& Masi, 2006). Additionally, more advanced stage at diagnosis and having tumors that are more aggressive are also included 
(Blackman \& Masi, 2006); however, there is little examination as to why these differences in tumor characteristics are observed in those who are ethnic minorities. Blackman and Masi (2006) believe that the factors above all play a role in the ethnic differences observed in cancer outcomes. It is suggested that by implementing interventions from the various perspectives (i.e., patient, provider, and health system) disparities may decline.

Associations with neighborhood stress. Research has shown that there is an interaction between individual and neighborhood SES with regard to mortality (Winkleby, Cubbinm \& Ahn, 2006). It is suggested that individuals with low individual SES living in a neighborhood with high SES have the highest all-cause mortality rates, presumably due to not having full access to resources in their neighborhood because of their personal SES (Winkleby et al., 2006). However, specifics regarding mortality due to cancer were not explored in the aforementioned study. It has been shown that individuals living in poverty areas and who are younger, age 25 through 54, are statistically more likely to die from cancer than those living in non-poverty areas; however, this relationship was not observed for individuals aged 55 to 74 (Waitzman \& Smith, 1998). In a sample of women with ovarian cancer, neighborhood disadvantage was shown to be related to higher tumor grade and neighborhood affluence was shown to be related to more advanced tumor stage at diagnosis (Peterson et al., 2014). In a study of colorectal and head and neck cancer patients in Taiwan, those with low individual SES living in disadvantaged neighborhoods has the highest mortality, as did those with breast and lung cancer with low individual SES living in advantaged neighborhoods (Chang et al., 2012). 
Interestingly, the importance of researching the neighborhood effect on mortality has been emphasized as larger effects are observed with mortality rather than morbidity (Ellen et al., 2001). This is due to the notion that there are only modest differences across communities concerning specific diseases; however, the disease trajectory is significantly informed by one's neighborhood environment (Ellen et al., 2001). Mixed results have been shown regarding mortality and neighborhood stress, and it appears that individual income, and possibly the interaction with access to care and resources may help to explain these differences. Given the significant role that neighborhood stress plays on the health and disease processes of an individual, and knowing the link between stress and cancer progression and mortality, examining these associations is crucial.

Associations with cancer-related stress. Limited research has been completed concerning cancer-related stress and cancer progression and survival. Among a sample of individuals with various types of cancer, avoidance but not intrusive thoughts were shown to be predictive of disease progression one year after diagnosis (Epping-Jordan, Compas, \& Howell, 1994). These findings seem intuitive in that those who are avoidant may not follow through with treatments or may not present to their physicians promptly when symptoms of recurrence are evident. Therefore, there appear to be strong implications for cancer-related stress regarding tumor progression and survival, which warrant additional exploration in the hopes of better understanding the consequences of cancer-related stress.

Physiological Disruption and Medical Outcomes of Cancer (Figure 1 Arrow C) Associations with circadian disruption. 
Rest/activity rhythms. Research has shown that disruption of circadian rhythms can be linked to progression of tumors as well as early mortality in cancer patients. A literature review indicated that clock genes influence cell cycles in mammary epithelium, which can lead to an improper division of the cells, leading to more aggressive breast tumors (Blakeman, Williams, Meng, \& Streuli, 2016). In a murine model, ablation of the SCN resulted in the loss of circadian rhythmicity, which was associated with faster tumor growth than in the control mice, which in turn lead to earlier mortality (Filipski et al., 2009). Additionally, mice with tumors had increased tumor growth and shorter survival when light/dark cycles disrupted circadian rhythms (Li \& Xu, 1997). Among patients with metastatic colorectal cancer, rest/activity rhythms have been shown to be significantly related to survival (Innominato et al., 2009; Lévi et al., 2014; Mormont et al., 2000). Additionally, sleep disruption was significantly predictive of survival in women with advanced breast cancer (Palesh et al., 2014). Given the demonstrated rest/activity rhythm relationships with survival in colorectal cancer patients, it would be interesting to see if this relationship generalizes to other cancer populations. Additionally, determining whether rest/activity rhythms, when measured at various time points throughout the cancer treatment trajectory (e.g., presurgically, during adjuvant treatment, and during survivorship), influence survival is worthy of exploration.

Hypothalamic pituitary adrenal (HPA) axis. Women with more advanced stage ovarian cancer had higher levels of salivary cortisol in the morning and afternoon compared to women with early stage ovarian cancer (Weinrib et al., 2010). Additionally, when comparing cortisol levels of women with ovarian cancer to healthy controls, it has been observed that afternoon and evening salivary cortisol is significantly higher in 
women with ovarian cancer (Weinrib et al., 2010). The afternoon cortisol levels were not significantly higher in women with ovarian cancer compared to those with benign disease; however, the evening cortisol levels were significantly different (Weinrib et al., 2010). With regard to morning cortisol levels, no significant differences were observed between any of the three groups (i.e., ovarian cancer, benign disease, healthy controls; Weinrib et al., 2010). Among women with metastatic breast cancer, those with more severe metastatic spread had higher mean salivary cortisol levels; however, no significant differences were observed in mean cortisol levels of any samples collected throughout each day between women with breast cancer and healthy controls (Abercrombie et al., 2004).

Diurnal cortisol slopes and cancer has also been explored. Flatter diurnal salivary cortisol slopes were observed in women with cancer compared to healthy controls; however, no significant diurnal salivary cortisol slope differences were found in women with more severe metastatic spread (Abercrombie et al., 2004). Among women with ovarian cancer, no significant differences were observed between those with early or advanced stage cancer (Weinrib et al., 2010).

Diurnal salivary cortisol slope was associated with mortality, such that those with earlier mortality had flattened slopes, which is indicative of a lack of diurnal variation in cortisol (Sephton et al., 2013). Additionally, flattened cortisol slopes were associated with advanced stage lung cancer, as well as poorer performance status (Sephton et al., 2013). This study also explored CAR and diurnal area under the curve (AUC); however, no association was observed with these cortisol variables and survival (Sephton et al., 2013). 
Among those with metastatic renal cell carcinoma, salivary cortisol slope was significantly associated with survival (Cohen et al., 2012). In women with metastatic breast cancer, similar results were observed, such that those with flattened diurnal salivary cortisol slopes had shorter length of survival (Sephton, Sapolsky, Kraemer, \& Spiegel, 2000). Furthermore, steeper salivary cortisol slopes were associated with metastases in the chest wall and adjacent lymph nodes rather than bone or distant organ metastases (Sephton et al., 2000). Additionally, it has been shown that increased nighttime cortisol levels and flattened cortisol slope were associated with early mortality in women with ovarian cancer (Schrepf et al., 2015). However, in a sample of individuals with metastatic colorectal cancer, neither serum total cortisol nor salivary free cortisol were predictive of survival over a period of four-year follow-up (Mormont, Bogdan, Cormont, Touitou, \& Levi, 2002).

Clinical human research has shown that those with cancer have greater mean cortisol levels compared to controls and those with benign disease and advanced cancer have higher cortisol levels that those with early stage cancer. Those with cancer also have flatter slopes compared to healthy controls. Furthermore, in differing cancer types flattened cortisol slopes have been shown to be associated with early mortality. Literature reviews have demonstrated that circadian disruption of endocrine rhythms exists in patients with cancer, and this dysregulation plays a vital role in tumor burden and cancer progression (Antoni et al., 2006; Eismann et al., 2010). Specifically, glucocorticoids may promote cancer progression through angiogenesis, invasion, migration, and apoptosis (Antoni et al., 2006). 
Associations with immune function. Immune parameters may act to suppress or promote the growth of tumors. Inflammation tends to play a vital role in the growth and spread of cancer and the interplay between tumor promoting, and tumor suppressing immune factors help in the determination of the outcome of the tumor (Zamarron $\&$ Chen, 2011). High peripheral levels of VEGF have been shown to be indicative of early mortality in hepatocellular carcinoma (Jin-no et al., 1998) and gastric cancer (Karayiannakis et al., 2002). MMP-9 plays a role in the metastasis of cancer, particularly elevated levels are associated with rapid progression, secondary metastasis, and poorer survival (Deryugina \& Quigley, 2006). Either disruption or loss of TGF- $\beta$ signaling leads to either migration, invasion, and metastasis or rapid growth and metastasis, respectively (Derynck, Akhurst, \& Balmain, 2001).

A specific genetic polymorphism of IL-1 $\beta$ leads to significantly higher production of IL-1 $\beta$ and shorter survival in a group of individuals with pancreatic cancer (Barber, Powell, Lynch, Fearon, \& Ross, 2000). TNF- $\alpha$ is involved in all processes of the progression of tumors (Balkwill, 2006). IL-6 serves to enhance growth and progression of tumors, as well as influences survival (Grivennikov et al., 2010). Higher IL-6 levels have been shown to be associated with a greater number of metastatic sites in women with breast cancer (Salgado et al., 2003) and are associated with poorer prognosis in both lung and breast cancer patients (Hodge, Hurt, \& Farrar, 2005). Increases in IL-6 may be due in part to increased production of IL-6R (Scambia et al., 1995).

Expression of MCP-1 plays a role in the histologic grade of cancers, such that MCP-1 negative tumors tend to be well-differentiated (Valković, Lučin, Krstulja, DobiBabić, \& Jonjić, 1998). Additionally, IL-12 and IFN- $\gamma$ may play a role in the progression 
of tumors (Mantovani et al., 2008). Research has shown relationships between immune function and tumor characteristics, progression, and mortality. Therefore, continued research may be beneficial in further understanding the relationship between immune function and the progression of cancer, as well as survival. Exploring relationships in individuals with active tumors prior to treatment allows for better understanding of the role that cancer plays in associations with immune disruption, compared to treatment effects.

\section{Summary and Hypotheses}

Health disparities, and more specifically, differences in cancer outcomes, are increasingly reported both for ethnicity and neighborhood stress-related variables. Additionally, research has shown a strong relationship between psychosocial stress, physiological disruption, and disease progression, particularly within a cancer population. However, life context (e.g., ethnicity and neighborhood-related stressors) has rarely been explored within this framework and little research has explored relationships between psychosocial stress and physiological disruption among individuals with cancer.

Two specific aims were explored in this dissertation: The first explored the relationships between ethnicity, neighborhood stress, and cancer-related stress in a group of women with breast cancer. The second aim explored the relationships between psychosocial stress, physiological disruption, and medical outcomes of cancer (progression-free survival time).

Aim 1. Associations between psychosocial stress measures.

Hypothesis 1A. Ethnicity and neighborhood will be significantly and positively correlated with one another (Figure 2, Arrow A). 

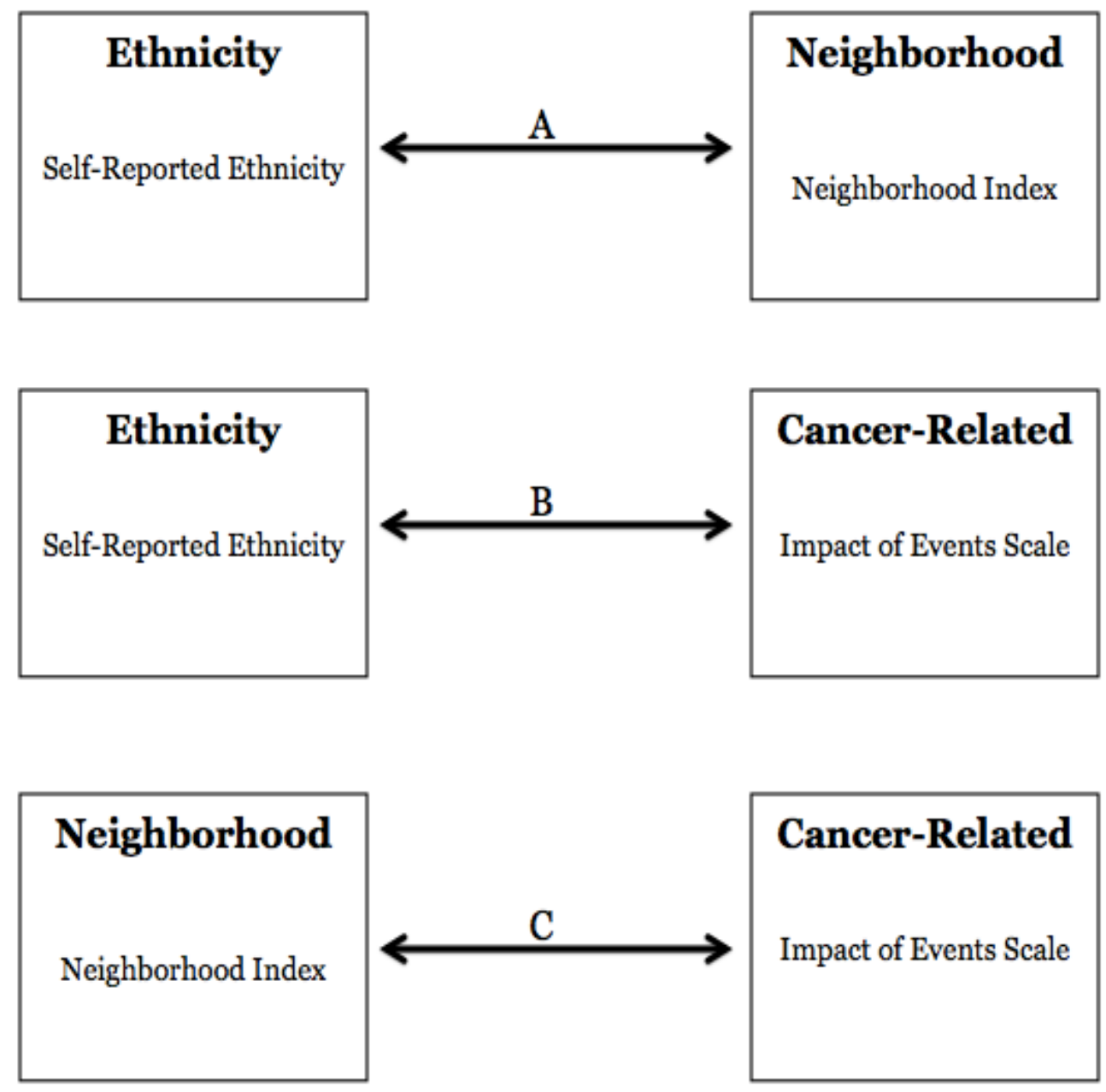

Figure 2. Aim 1, associations between psychosocial stress measures.

Hypothesis $1 B$. Ethnicity and cancer-related stress will be significantly and positively correlated with one another (Figure 2, Arrow B).

Hypothesis 1C. Neighborhood socioeconomic stress and cancer-related stress will be significantly and positively correlated with one another (Figure 2, Arrow C).

Aim 2. Associations between psychosocial stress, physiological disruption, and medical outcomes of cancer (progression-free survival). 
Hypotheses 2A. Tests of hypotheses 2 will explore associations of psychosocial stress (i.e., ethnicity, neighborhood stress, and cancer-related stress) with physiological disruption (i.e., circadian, endocrine, and immune; Figure 1, Arrow A).

Hypothesis 2A.1. African American ethnicity will be independently associated with greater circadian disruption, endocrine disruption, and immune disruption (Figure $3)$.

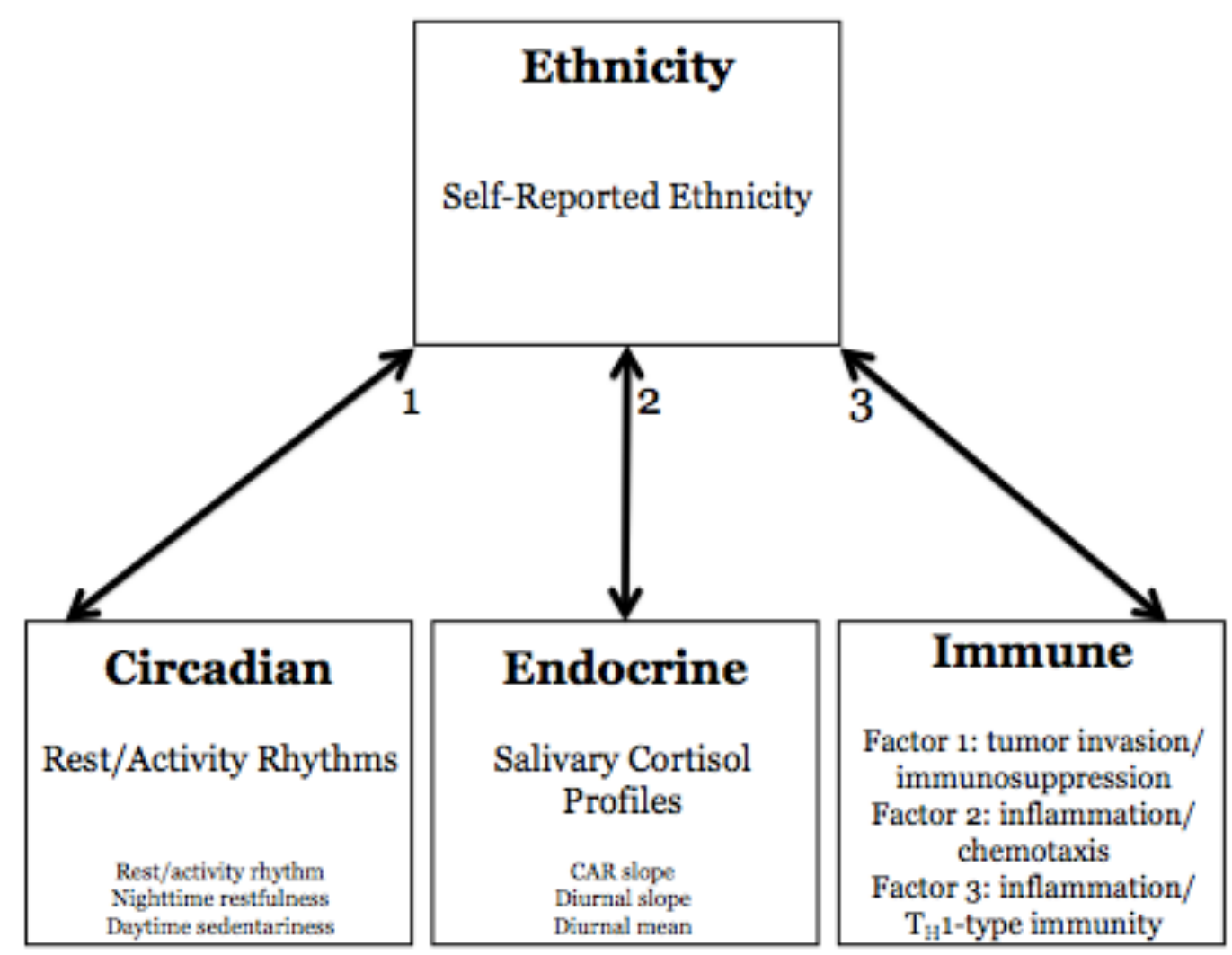

Figure 3. Hypothesis 2A.1, associations of ethnicity with physiological disruption. 
Hypothesis 2A.2. Neighborhood stress will be independently associated with greater circadian disruption, endocrine disruption, and immune disruption (Figure 4).

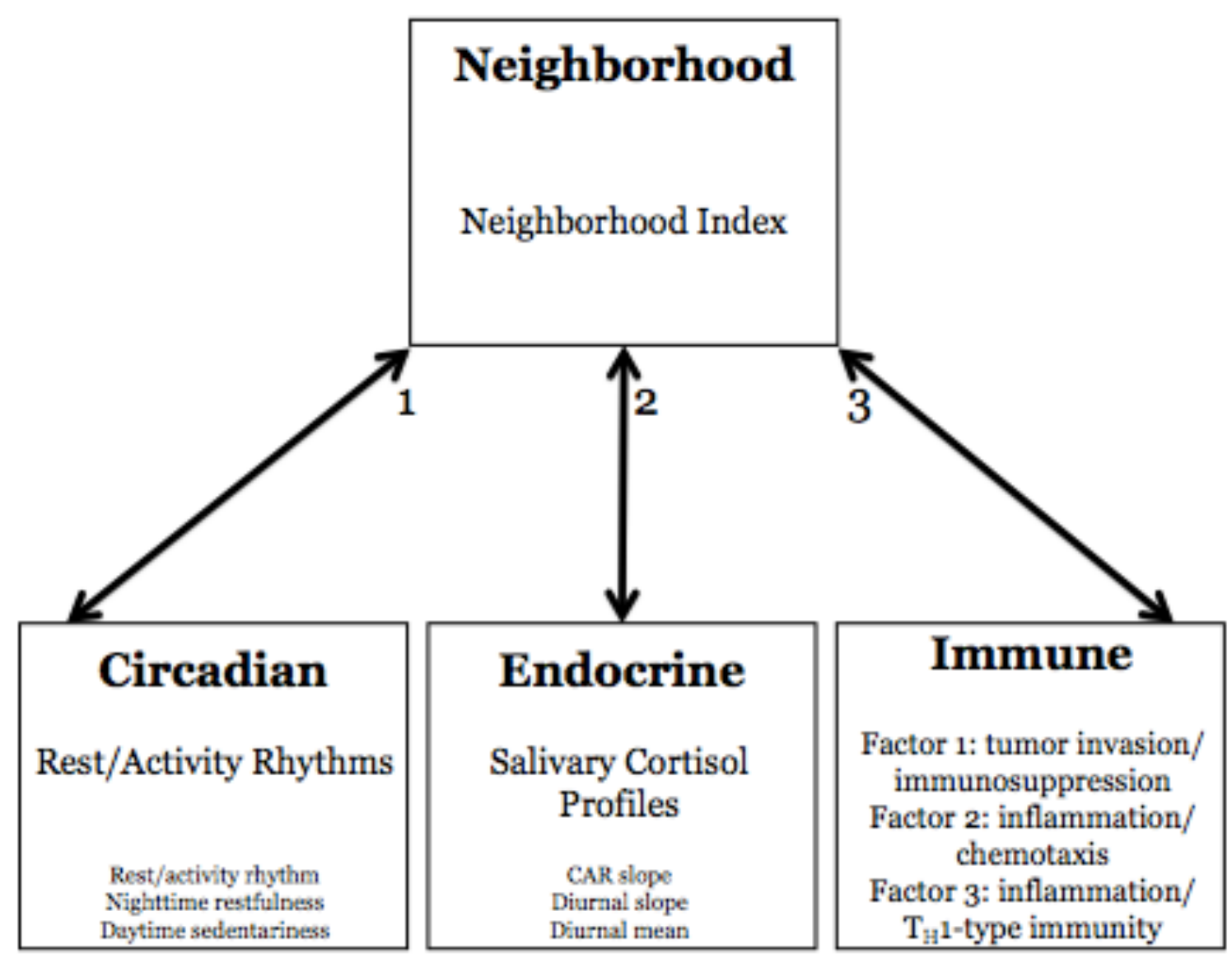

Figure 4. Hypothesis 2A.2, associations of neighborhood stress with physiological disruption. 
Hypothesis $2 A .3$. Cancer-related stress will be independently associated with greater circadian disruption, endocrine disruption, and immune disruption (Figure 5).

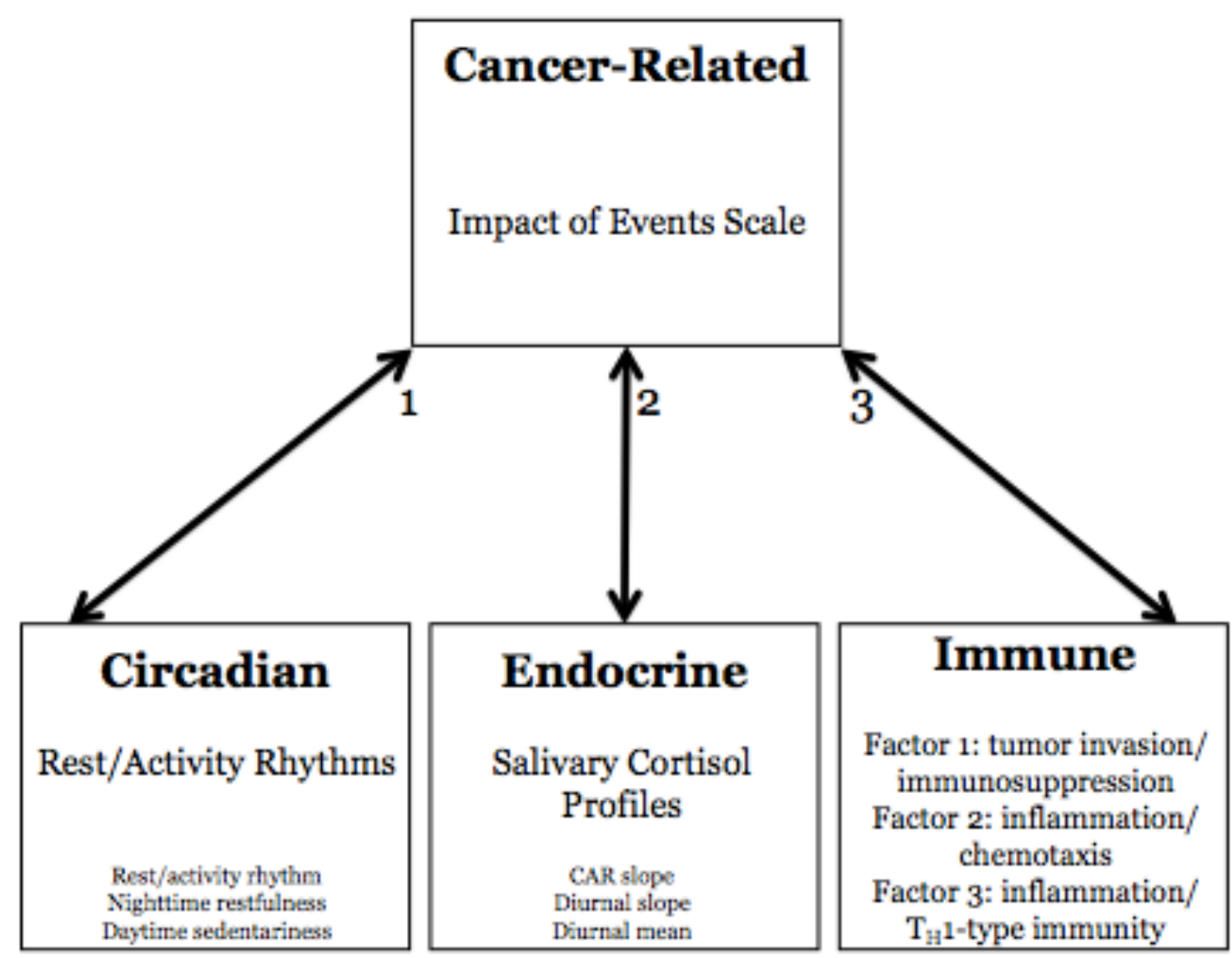

Figure 5. Hypothesis 2A.3, associations of cancer-related stress with physiological disruption. 
Hypotheses 2B. Tests of hypotheses 2B will explore associations of psychosocial stress with medical outcomes of cancer (progression-free survival time; Figure 1, Arrow B).

Hypothesis 2B.1. African American ethnicity will be independently associated with shorter breast cancer progression-free survival time (Figure 6, Arrow 1).

Hypothesis 2B.2. Neighborhood stress will be independently associated with shorter breast cancer progression-free survival time (Figure 6, Arrow 2).

Hypothesis $2 B .3$. Cancer-related stress will be independently associated with shorter breast cancer progression-free survival time (Figure 6, Arrow 3).

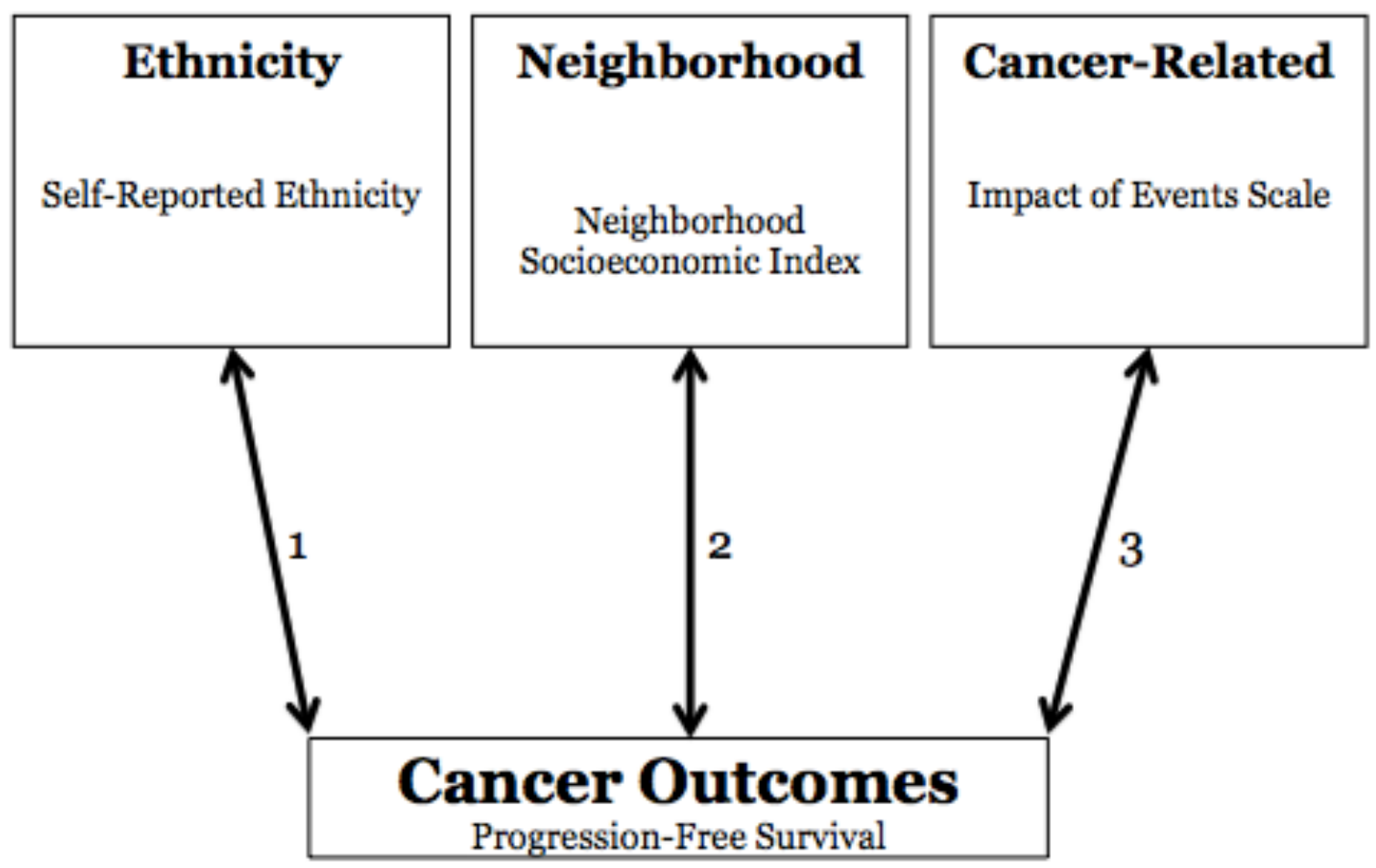

Figure 6. Hypotheses 2B, associations of psychosocial stress with medical outcomes of cancer (progression-free survival time). 
Hypotheses 2C. Tests of hypotheses $2 \mathrm{C}$ will explore associations of physiological disruption and medical outcomes of cancer (progression-free survival time; Figure 1, Arrow C).

Hypothesis 2C.1. Circadian disruption will be independently associated with shorter breast cancer progression-free survival time (Figure 7, Arrow 1).

Hypothesis 2C.2. Endocrine disruption will be independently associated with shorter breast cancer progression-free survival time (Figure 7, Arrow 2).

Hypothesis 2C.3. Immune disruption will be independently associated with shorter breast cancer progression-free survival time (Figure 7, Arrow 3).

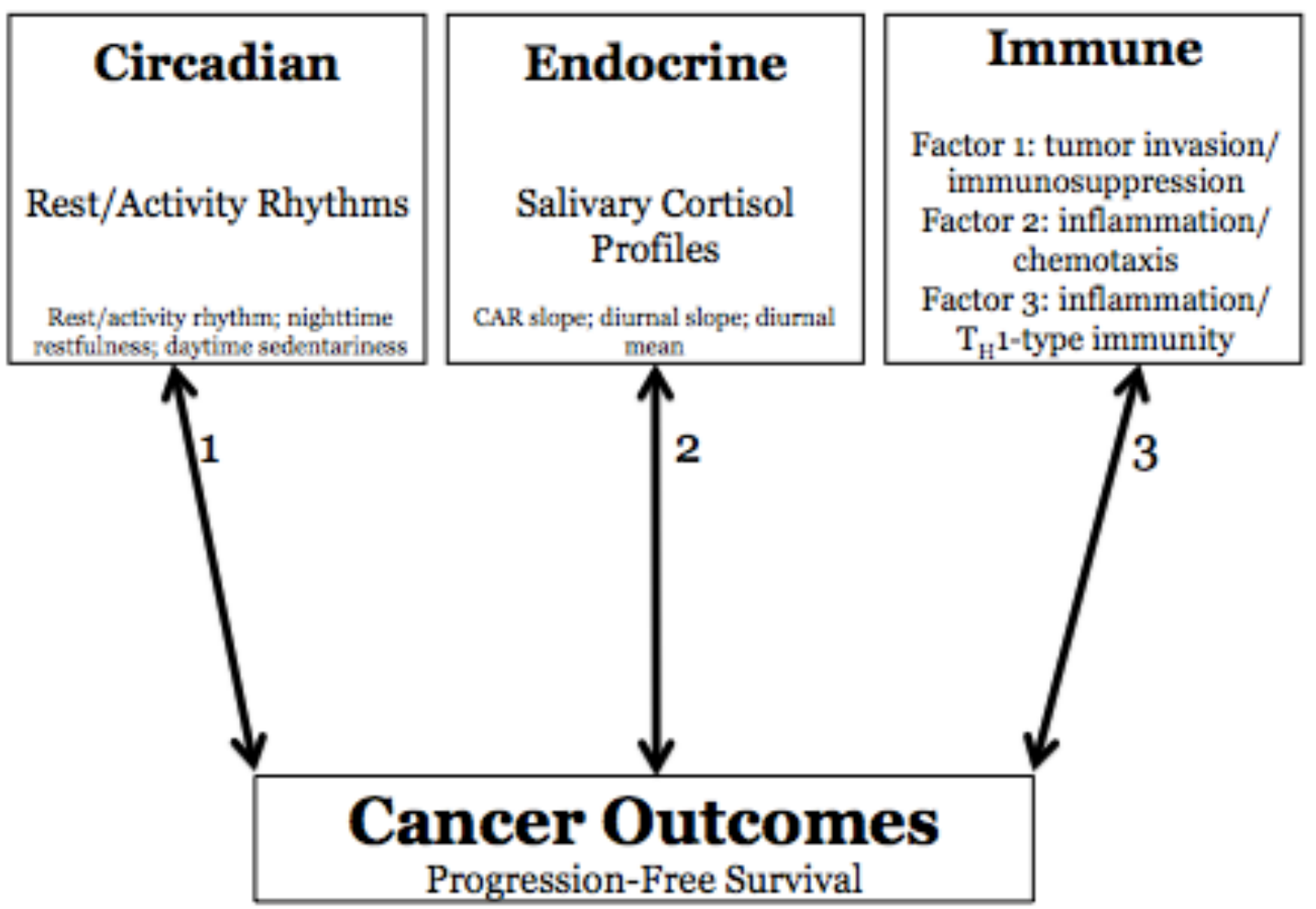

Figure 7. Hypotheses 2C, associations of physiological disruption with medical outcomes of cancer (progression-free survival time). 


\section{METHODS}

\section{Participants}

Participants in this study were recruited as part of a previous study titled "Stress, Coping and Sleep in Newly Diagnosed Women with Breast Cancer.” The study was designed to understand how a breast cancer diagnosis impacts stress and the ability to cope; therefore, participants were recruited as close as possible to their diagnosis of breast cancer. The prior studies that utilized the data tested hypotheses that are separate from those examined in this study. The prior studies explored relationships between daily ratings of cancer-related stress and coping with actigraphy and cortisol measures (Dedert et al., 2012) and the associations between social integration, support, and immune function (serum and stimulated; Cash et al., 2015).

Women diagnosed with primary, recurrent, or metastatic breast cancer who had not begun treatment (i.e., surgery, chemotherapy, or radiation) were eligible for enrollment according to a protocol approved by the University of Louisville Institutional Review Board. Inclusion criteria required that all participants be English speakers without active psychoses.

\section{Recruitment}

Recruitment personnel (i.e., graduate research assistants from Dr. Sandra Sephton's laboratory) were present in the clinic of Dr. Anees Chagpar, the referring 
surgical oncologist, to recruit eligible participants. Dr. Kelly McMasters also referred patients when Dr. Chagpar did not hold clinic. Recruitment occurred when the referring physician was in clinic, which occurred two days per week. Therefore, recruitment took place in two clinics, one day at the University Surgical Associates clinic and the other day at the James Graham Brown Cancer Center. Medical records were examined to determine which patients were possibly eligible for recruitment. Additionally, clinic personnel would help alert research assistants to potentially eligible patients. Eligible patients were introduced to the study by surgical oncologist, Dr. Anees Chagpar after a biopsy to confirm diagnosis of breast cancer was completed. Patients who were not referred during their visit to the clinic were invited to enroll by phone.

Initial recruitment occurred from May 2005 through June 2007. During this wave of recruitment, 49 participants were enrolled and provided at least partial data on selfreport questionnaire, actigraphy, saliva, and immune measures. Complete serum immune data was obtained from 30 participants. Incomplete immune data occurred due to inability to complete a blood draw. A second wave of recruitment from December 2008 to February 2009 occurred to increase the sample size. An additional 11 participants were enrolled, for a total of 60 participants.

During both waves of recruitment, 91 potentially eligible patients were referred to the study. Five were introduced to the study; however, due to their time constraints were not enrolled in the clinic and were unable to be reached by phone. Response burden and/or time constraints were the reasons that 22 potential patients declined to participate. One participant was not enrolled because of difficulties understanding data collection procedures and low English proficiency. Additionally, one patient felt she lived too far 
away to participate, one patient was scheduled for treatment too soon to complete data collection, and one patient declined because she was uncomfortable consenting to review of medical records. Of the 60 participants enrolled, one participant provided no data due to an illness, one provided no data due to time demands of having family visit, and one was unable to complete collection due to increased time constraints following enrollment. Seven participants provided partial self-report data, two participants experienced problems with actiwatch recording, which prevented actigraphy data collection, one participant did not provide saliva samples, and seven participants did not provide blood samples. Overall, there was enough data provided by 57 participants to be included in analyses.

\section{Data Collection Procedures}

A graduate research assistant obtained informed consent and research authorizations per requirements of HIPAA. Study rationale and procedures were explained and data collection materials were provided to participants. Participants were provided with two questionnaire packets, one that contained demographic and self-report measures and one that contained daily items to assess variables related to actigraphy (e.g., time going to bed and waking up) and cortisol (e.g., alcoholic and caffeinated beverage consumption). A wrist-worn actiwatch was placed on the participant's wrist at this time and information was provided regarding actiwatch care (e.g., the watch was not waterresistant) and an opportunity to ask questions. The participants were asked to wear the actiwatch for the coming three days. Participants were also provided with a saliva collection kit, as well as a demonstration of saliva collection and an opportunity to 
practice and ask questions. Participants were asked to collect four saliva samples per day over the next three days. Participants had blood drawn by a trained phlebotomist.

Neighborhood stress and progression-free survival data were collected at a later date by a research assistant. The neighborhood stress data were gathered via the United States Census. Progression-free survival data were obtained from the Kentucky Cancer Registry (KCR).

\section{Measures}

Demographics. Participants completed a background questionnaire, which assessed various demographic variables, including age, ethnicity, religious affiliation, education, employment, household income, and living situation. Additionally, the background questionnaire also assessed medical history and current medications that the participant was taking.

\section{Psychosocial Stress Measures}

Ethnicity. Self-reported ethnicity was a categorical variable for which participants indicated which of the following ethnicities they identified with most: non-Hispanic (NH) White, African American, Hispanic, Asian American, Native American, and other. Out of respect for potential ethnic/cultural differences, analyses only included those reporting their ethnicity as NH White or African American.

Neighborhood stress. Participant addresses were provided as a part of the demographic data collection. Data regarding each participant's neighborhood status was obtained from the United States Census Bureau's 2010 census data using the participant addresses. For each participant, the following four variables were the only neighborhood data extracted from www.census.gov's American FactFinder application: percentage of 
households below the poverty line, median household income, percentage of singleparent homes, and percentage of renter-occupied housing in the neighborhood. The data was obtained at the census tract level, which is a small subdivision of a county. Sarah Ehresman, research manager for the Kentucky State Data Center, served as a consultant for obtaining data from the American FactFinder application by providing information regarding geocoding and demonstrating how to use the software.

An index of neighborhood stress was created with principal components analysis (PCA), as we believe that one factor score would be more representative of the cumulative neighborhood stress, rather than variables used individually. Additionally, a PCA was chosen in order to minimize the number of statistical tests that were run. To conduct a PCA, first neighborhood stress variables were log-transformed due to skewness, then standardized into z-scores since the four variables of neighborhood stress are not the same scale of measurement (i.e., percentages and median values). Then factor scores were created for each individual case with the regression method, as we expected the variables to be correlated with one another, in SPSS using loadings from the PCA with varimax rotation. Factor scores are the sum of the product of the factor loading and the value of the individual variable of neighborhood stress. Factor scores were used as the independent variable, neighborhood stress, in analyses.

Cancer-related stress. The Impact of Events Scale (IES) is a measure of a participant's current amount of distress related to their cancer (Horowitz et al., 1979). The IES measures both intrusive thoughts and avoidance associated with the diagnosis and treatment of cancer. The 15 -item measure asked that participants rate the frequency of the occurrence of each item over the past seven days on a four-point Likert scale 
ranging from "not at all" to "often." The test-retest reliability of the total score is 0.87 , and for the intrusions and avoidance subscales 0.89 and 0.79 , respectively (Horowitz et al., 1979).

Scores greater than 19 on the IES total score are of high clinical concern (Horowitz, 1982); however, this is an arbitrary cutoff and does not indicate a specific clinical diagnosis (Joseph, 2000). Scores of 35 or above may be clinically significant for the presence of symptoms of PTSD (Haagsma et al., 2012; Wohlfarth, van den Brink, Winkel, \& ter Smitten, 2003).

While there are no specific African American norms for the IES, it has been used in studies that recruited both NH White women and African American women with breast cancer (Cordova et al., 1995), as well as studies of only African American women with breast cancer (Taylor et al., 2003). Therefore, it appears that this measure is appropriate to use in exploring cancer-related stress in both NH White and African American populations.

\section{Circadian rhythms.}

Rest/activity rhythms. Circadian rest/activity rhythms were measured via wristworn movement recording devices (Ancoli-Israel et al., 2003; MicroMini-Motionlogger; Ambulatory Monitoring Systems, Inc, Ardsley NY). Over a consecutive period of four nights and three days, the participants' body movements were recorded. Participants were asked to remove the actigraph watch when doing activities that would cause the device to get wet. Sleep intervals for each participant were set by integrating self-reported times for sleep onset and awakening with actigraphy data, with preference given to actigraphy data when discrepant. 
Commercially available software was utilized to score all one-minute epochs and the following measures were obtained: rest/activity rhythm measured by the autocorrelation coefficient of activity measured across 24 hours, and two dichotomy indices comparing daytime activity level to the activity level while the participant was in bed (i.e., daytime sedentary behavior, nighttime restfulness). Rest/activity rhythm, a measure of circadian consistency or the similarity of patterns of activity across days, was calculated by correlating the epochs of activity during the first day of collection with the epoch of activity at the same time on the other days. Similar activity levels evidence a strong circadian rhythm at similar times of day, which yields a high rest/activity rhythm. The dichotomy indices compare activity when in bed with activity when out of bed. The dichotomy index for inactivity out of bed, which is daytime sedentary behavior, is the percentage of activity counts out of bed that fall below the median level of activity counts when the participant is in bed. The dichotomy index for inactivity in bed, indicative of nighttime restfulness, is the percentage of activity counts in bed that is below the median level of activity counts when the participant is out of bed. A higher dichotomy index indicates more frequent inactivity. Therefore, greater daytime sedentariness is consistent with circadian disruption, whereas greater nighttime restfulness is consistent with strong circadian rhythmicity.

Salivary cortisol profiles. Participants provided a total of 12 saliva samples, specifically three consecutive days at waking, 30 minutes after waking $(+30)$, at four in the afternoon, and immediately prior to bed. Medication Event Monitoring System (MEMS) bottles and caps (Aardex, 2001) were used to store cotton saliva collection swabs and recorded the exact date and time the bottle was opened. Participants were 
asked not to eat, drink, brush their teeth, use mouthwash, chew gum, or smoke for 30 minutes prior to saliva collection. Upon completion of data collection, the saliva samples were centrifuged, aliquoted, and stored at $-80^{\circ} \mathrm{C}$.

Assays were conducted using an enzyme immunoassay (EIA) developed for use in saliva (Salimetrics, Inc., State College, PA). The sensitivity of the assay was 0.003 $\mu \mathrm{g} / \mathrm{dL}$. To ensure accuracy between assay plates, assay reliability is measured as the coefficient of variation (CV; mean divided by standard deviation). CVs for low and high controls should be less than $15 \%$ and 5\%, respectively. For these data, the inter-assay CV was $10.45 \%$ for low controls and $4.03 \%$ for high controls.

The MEMS and self-reported saliva collection times were highly correlated (Spearman's $r=.962$ ), which suggests that the participant reports of collection times were reliable (Kraemer et al., 2006). MEMS collection times were used when there were discrepant reports. Additionally, actigraphy data were reviewed to examine for discrepancies between waking and bedtime self-reports, with preference given to actigraphy data. Collection time and cortisol values greater than four standard deviations from the mean were considered outliers and deleted ( $\mathrm{n}=9$ samples, $1.3 \%)$. Also, nonadherence to the collection time protocol resulted in deletion of the cortisol values $(\mathrm{n}=$ 83, 12.3\%). See Dedert et al., 2012 for further details.

Cortisol values were log-transformed. The cortisol awakening response (CAR) was calculated as the unstandardized beta of natural log-transformed cortisol regressed on collection time and included the waking and +30 min samples. Diurnal cortisol slope was calculated with the waking, 4:00 o'clock, and bedtime samples, excluding the +30 min 
sample, as the unstandardized beta of log-transformed cortisol regressed on collection time. Diurnal mean cortisol was calculated using all 12 log-transformed values.

Immune function. A trained phlebotomist drew blood using an empty Vacutainer $^{\mathrm{TM}}$ tube. Blood was collected as close to study entry as possible, always before surgical treatment. Circadian patterns of immune molecule release in system circulation occur (Kronfol, Nair, Zhang, Hill, \& Brown, 1997); therefore, a restriction in the timing of the blood draw was attempted as much as possible within the limits of the schedule at the clinic where recruitment occurred. The majority of blood draws took place in the early afternoon $(\mathrm{M}=1: 15 \mathrm{PM}$, median = 1:32 PM $)$ with 40 of the samples being collected in the afternoon and 11 in the morning (Cash et al., 2015).

When the blood draw was completed, whole blood samples from the nonanticoagulant tube were centrifuged to separate serum from red blood cells. Serum, which gives the measure of the general level of immune parameters present, was pipetted, and aliquots were immediately frozen at $-80^{\circ} \mathrm{C}$ until assayed.

Cytokines were analyzed using electrochemiluminescent quantitative arrays from MesoScale Discovery (Gaithersburg, MD). Each sample was analyzed in duplicate according to the manufacturer protocol. Assay plates were imaged on a SECTOR Imager 2400A and data were analyzed using Discovery Workbench software (MesoScale). In addition to running all manufacturer specified controls, a pooled internal control was used, and validation and maintenance of assay quality were assured by running additional samples of the pooled control "spiked" with known amounts of analytes to fall within specific regions of the standard curve. 
Factor analyses were previously completed to determine groupings of the 10 immune parameters with a data-driven approach (Cash et al., 2015). The factor score from each of the three clusters that emerged from the exploratory factor analysis (EFA) was utilized in analyses. Factor 1 included VEGF, MMP-9, and TGF- $\beta$ and is indicative of tumor invasion and immunosuppression. Factor 2 included proinflammatory and chemotactic markers, including IL-1 $\beta$, IL-6R, TNF- $\alpha$, and MCP-1. Factor 3 included IL6 , IL-12, and IFN- $\gamma$, measures of proinflammation and $\mathrm{T}_{\mathrm{H}}-1$ type processes.

Medical outcomes of cancer (progression-free survival time) Consent was obtained in order to access patient data from the KCR for determination of recurrent status or survival in future analyses. Progression-free survival data were obtained from KCR approximately five years after the last participant was recruited.

Medical records review. Functional status, measured with the Karnofsky Performance Status scale (Schag, Heinrich, \& Ganz, 1984), and information regarding medications were collected during the initial interview. Medical charts were reviewed after surgery in order to obtain accurate data on treatment choice and surgical pathology information, including cancer stage and histology.

Control variables. The control variables utilized were age at time of cancer diagnosis and cancer stage for all analyses, as both are associated with variance in the speed of the progression of the tumor, as well as the level of associated psychosocial stress (Andersen, Kiecolt-Glaser, \& Glaser, 1994; Antoni et al., 2006). Additionally, yearly income was controlled in all analyses as recruitment took place in two different clinics, under the same surgical oncologist, where income varied significantly (Cash et al., 2015; Dedert et al., 2012). 


\section{Statistical Analyses}

Data reduction and approach to analyses. All questionnaire data were manually entered, cleaned, and examined for abnormal responses. Data were analyzed using an exploratory/hypothesis generating approach, rather than hypothesis testing, in order to maximize breadth of investigation to better understand the data provided by this sample of presurgical breast cancer patients. Exploratory analyses are useful in elucidating relationships that are not yet fully understood. However, there are drawbacks to exploratory research, including that a significant number of analyses are likely to yield some significant relationships that are spurious. Therefore, it is necessary to be cautious in the interpretation of exploratory research. By hypothesis generating, a large number of analyses were run in order to better understand and characterize associations within the sample and to inform future directions, while taking care not to generalize outside this sample.

\section{Tests of hypotheses.}

Preliminary Analytical Procedures. The assumption of normal distribution of variables was evaluated by examining boxplots, histograms, and the skewness statistic. Variables that were non-normally distributed were log-transformed. Additionally, for variables in Hypothesis 1 that did not exhibit a normal distribution, Spearman correlations will be used, as opposed to Pearson correlations.

Hypothesis 1A. Bivariate correlations were utilized to test the correlations between ethnicity and neighborhood stress. A total of one correlation was used to test hypothesis $1 \mathrm{~A}$. 
Hypothesis 1B. Bivariate correlations were utilized to test the correlations between ethnicity and cancer-related stress. A total of one correlation was used to test hypothesis $1 \mathrm{~B}$.

Hypothesis 1C. Bivariate correlations were utilized to test the correlation between neighborhood stress and cancer-related stress. A total of one correlation was used to test hypothesis $1 \mathrm{C}$.

Hypothesis 2A.1. Hierarchical linear regressions were performed to determine the relationship between ethnicity and physiological disruption. The theoretically-derived control variables, age at diagnosis, stage, and income, were entered in the first block, ethnicity was entered in the second block, and each physiological disruption variable was entered separately as the outcome. A total of nine regressions were used to test hypothesis 2 A.1.

Hypothesis 2A.2. Hierarchical linear regressions were run to determine the relationship between neighborhood stress and physiological disruption. Analyses were completed in the same manner as hypothesis $2 \mathrm{~A} .1$; however, neighborhood stress was entered into the second block as the independent variable. A total of nine regressions were used to test hypothesis $2 \mathrm{~A} .2$.

Hypothesis 2A.3. Hierarchical linear regressions were conducted to determine the relationship between cancer-related stress and physiological disruption. Cancer-related stress was entered as the independent variable in block two, with all other variables treated in the same manner as hypothesis $2 \mathrm{~A} .1$. A total of nine regressions were used to test hypothesis $2 \mathrm{~A} .3$. 
Hypothesis 2B.1. Survival analyses estimate the likelihood of an event occurring at a certain time. Participants were tracked over a period of time, with the focus on the time at which the event (i.e., recurrence/death or last contact for those who have not experienced recurrence or death) occurred. Therefore, time refers to the amount of time from either date of initial diagnosis or date of study entry until the event. In Cox proportional hazards analyses, the dependent variable is composed of two parts, the event status (i.e., recurrence/death or no recurrence/death) and the time to the event.

A two-tailed Cox proportional hazards model was used to test the effects of ethnicity on progression-free survival time, measured from the date of initial diagnosis and the date of study entry. The two time variables were entered, independently, into the time box, the event (i.e., progression-free survival status) was entered into the status box, and the independent variable, ethnicity, was entered into block one. For significant associations, additional tests will be completed as a check for potential confounding variables and their effects on the findings (Kraemer et al., 2001; Kraemer et al., 2008). A total of two regressions were used to test hypothesis $2 \mathrm{~B} .1$

Hypothesis 2B.2. The Cox proportional hazards model was used in survival analyses testing the prognostic value of neighborhood stress on progression-free survival time. The analyses were completed similarly to hypothesis $2 \mathrm{~B} .1$; however, for this hypothesis the independent variable was neighborhood stress. A total of two regressions were used to test hypothesis 2B.2.

Hypothesis 2B.3. The Cox proportional hazards model was used in survival analyses testing the prognostic value of cancer-related stress on progression-free survival time. For these analyses, the independent variable was cancer-related stress, and the 
analyses were conducted in the same way as hypothesis $2 \mathrm{~B} .1$. A total of two regressions were used to test hypothesis 2 B.3.

Hypothesis 2C.1. The Cox proportional hazards model was used in separate survival analyses testing the prognostic value of autocorrelation coefficient, daytime sedentary behavior, and nighttime restlessness on progression-free survival time. Analyses were completed in the same manner as hypothesis $2 \mathrm{~B} .1$; however, in these analyses, the independent variables were variables of circadian disruption (i.e., autocorrelation coefficient, daytime sedentary behavior, and nighttime restlessness). A total of six regressions were used to test hypothesis $2 \mathrm{C} .1$.

Hypothesis 2C.2. The Cox proportional hazards model was used in separate survival analyses testing the prognostic value of CAR, diurnal cortisol slope, and mean cortisol on progression-free survival time. Hypothesis 2C.2 looks at CAR, diurnal cortisol slope, and mean cortisol as individual predictors and, as in hypothesis $2 \mathrm{~B} .1$, all other variables were treated in a similar fashion. A total of six regressions were used to test hypothesis 2 C.2.

Hypothesis 2C.3. The Cox proportional hazards model was used in separate survival analyses testing the prognostic value of three immune factors on progressionfree survival time. The analyses for hypothesis 2 C. 3 were conducted similarly to hypothesis 2 B.1; however, in these analyses the independent variables were the three immune factors. A total of six regressions were used to test hypothesis 2 C.3.

Power Analyses. Given that this study utilized previously collected data, post hoc power analyses were conducted to determine that we have adequate power with the sample size that was recruited to complete analyses. Power to detect effects is based on 
calculations using G*Power statistical software (Faul, Erdfelder, Lang, \& Buchner, 2007) for correlations and hierarchical linear regressions and XLSTAT-Power (Addinsoft, 2014) for Cox proportional hazards models. For hypothesis 1 little research was available for the specific stressors that are included in analyses; therefore, since the profile of mood states total mood disturbance (POM-TMD) was not be used in any analyses, the correlation between the IES and POM-TMD was used to complete power analysis for this hypothesis. Estimates were set at 0.354 for effect size with a sample size of 57 and $\alpha=$ .05 , hypothesis 1 has $80 \%$ power to detect effects (Faul et al., 2007).

Very limited research has studied the associations that are explored in hypotheses 2A.1, 2A.2, and 2A.3 and no effect size could be found in the literature that was obtained. Therefore, estimates were set at a medium effects size of $\mathrm{f}^{2}=.15$. With a sample size of 57 and $\alpha=.05$, hypotheses 2A.1, 2A.2, and $2 \mathrm{~A} .3$ has $82 \%$ power to detect effects (Faul et al., 2007).

For hypothesis 2C.2 power analysis for the Cox regression was completed in the XLSTAT-Power program. With the event rate (i.e., recurrence/death) set at 50\% and the hazards ratio of 34867.43 , the standard deviation of cortisol slope 0.062 , and the correlation between days of survival since diagnosis and cortisol slope of -0.194 (Sephton et al., 2013), with a sample size of 57 and $\alpha=.05$, the power to detect effects was $100 \%$ (Addinsoft, 2014). Unfortunately, power analyses could not be calculated for hypotheses 2B.1, 2B.2, 2B.3, 2C.1, and 2C.3. While previous literature reports hazards ratios for analyses between ethnicity and survival (Chlebowski et al., 2005), neighborhood stress and survival (Waitzman \& Smith, 1998), psychological distress and survival (Hamer, Chida, \& Molloy, 2009), circadian disruption and survival (Lévi et al., 2014), and 
immune function and survival (Karayiannakis et al., 2002), the additional data needed to calculate power analyses for cox regression (i.e., standard deviation of the independent variable and the correlation between the independent and dependent variable) was not reported. 


\section{RESULTS}

\section{Sample Characteristics}

Sociodemographic characteristics. Study participants ranged in age from 21 to 79 years, with an average (SD) age of 52.32 (13.30) years. Participants were mostly married $(n=17 ; 30.9 \%)$, divorced and still single $(n=17 ; 29.1 \%)$, or widowed $(n=10$; 18.2\%). Additionally, many participants were living with their spouse $(n=15 ; 27.3 \%)$, with only their children $(n=11 ; 20.0 \%)$, or alone $(n=10 ; 18.2 \%)$ and about one third of participants were currently renting their home $(n=19 ; 35.8 \%)$.

The majority of participants had received a high school diploma $(n=36 ; 65.5 \%)$. Approximately $61 \%(n=31)$ of participants were below the poverty line, with the majority of participants indicating that their annual household income was less than $\$ 20,000(n=20 ; 39.2 \%)$ or ranging from $\$ 20,000-\$ 39,999(n=13 ; 25.5 \%)$. Nearly half of our participants were unemployed $(n=27 ; 47.4 \%)$ and nearly $10 \%(n=5)$ of participants did not have insurance. Additional and more detailed sociodemographic characteristics are provided in Table 1. 


\section{Table 1}

\section{Sociodemographic Characteristics}

\begin{tabular}{|c|c|c|}
\hline & Frequency & Percent \\
\hline \multicolumn{3}{|l|}{ Marital Status } \\
\hline Single & 7 & $12.7 \%$ \\
\hline Single, divorced & 16 & $29.1 \%$ \\
\hline Single, cohabitating & 1 & $1.8 \%$ \\
\hline Married & 17 & $30.9 \%$ \\
\hline Divorced, remarried & 3 & $5.5 \%$ \\
\hline Widowed & 10 & $18.2 \%$ \\
\hline Widowed, remarried & 1 & $1.8 \%$ \\
\hline \multicolumn{3}{|l|}{ Education } \\
\hline Middle School & 3 & $5.5 \%$ \\
\hline High School & 36 & $65.5 \%$ \\
\hline Associates/Technical & 5 & $9.1 \%$ \\
\hline Bachelors & 5 & $9.1 \%$ \\
\hline Masters & 4 & $7.3 \%$ \\
\hline Doctorate & 2 & $3.6 \%$ \\
\hline \multicolumn{3}{|l|}{ Income } \\
\hline Less than $\$ 20,000$ & 20 & $39.2 \%$ \\
\hline$\$ 20,000-\$ 39,999$ & 13 & $25.5 \%$ \\
\hline$\$ 40,000-\$ 59,999$ & 5 & $9.8 \%$ \\
\hline$\$ 60,000-\$ 79,999$ & 4 & $7.8 \%$ \\
\hline$\$ 80,000-\$ 99,999$ & 4 & $7.8 \%$ \\
\hline$\$ 100,000$ and above & 5 & $9.8 \%$ \\
\hline \multicolumn{3}{|l|}{ Employment } \\
\hline Currently Employed & 30 & $52.6 \%$ \\
\hline Not Currently Employed & 27 & $47.4 \%$ \\
\hline \multicolumn{3}{|l|}{ Living Situation } \\
\hline Living alone & 10 & $18.2 \%$ \\
\hline Living with spouse/partner only & 15 & $27.3 \%$ \\
\hline Living with spouse/partner and child(ren) only & 7 & $12.7 \%$ \\
\hline Living with child(ren) only & 11 & $20.0 \%$ \\
\hline Living with other relatives & 8 & $14.5 \%$ \\
\hline Living with non-relatives & 2 & $3.6 \%$ \\
\hline Other & 2 & $3.6 \%$ \\
\hline \multicolumn{3}{|l|}{ Own or Rent Home } \\
\hline Own & 34 & $64.2 \%$ \\
\hline Rent & 19 & $35.8 \%$ \\
\hline \multicolumn{3}{|l|}{ Poverty Line } \\
\hline Above & 20 & $39.2 \%$ \\
\hline Below & 31 & $60.8 \%$ \\
\hline \multicolumn{3}{|l|}{ Insurance Status } \\
\hline Private & 24 & $47.1 \%$ \\
\hline Public & 9 & $17.6 \%$ \\
\hline Other & 13 & $25.5 \%$ \\
\hline None & 5 & $9.8 \%$ \\
\hline
\end{tabular}


Clinical characteristics. The majority of participants had stage I $(n=25 ; 42.4 \%)$, primary $(n=52 ; 88.1 \%)$ breast cancer. Additionally, $77.4 \%(n=41)$ of participants had positive ER status, $75.0 \%(n=39)$ had positive PR status, and almost $90 \%(n=42)$ had Her2Neu negative status, which is reflective of the most common hormone status combination (Onitilo et al., 2009). Furthermore, eight participants (17.0\%) had triple negative status, for which there are fewer treatment options (Bauer et al., 2007) and is linked to shorter survival time (Onitilo et al., 2009). The primary treatment of choice was mastectomy $(\mathrm{n}=49 ; 94.2 \%)$, while the remainder chose lumpectomy. The Karnofsky Performance Status scale provides a rating of patient's functional status (Schag et al., 1984) with scores ranging from 100 (no evidence of disease or impairment from disease) to 0 (deceased). The majority of patients' functional status was rated as no evidence of disease or impairment from disease (i.e., Karnofsky $=100 ; \mathrm{n}=23 ; 44.2 \%$ ) or minor signs or symptoms of disease (i.e., Karnofsky $=90 ; n=20 ; 38.5 \%$ ), suggesting there was minimal to no impairment due to disease symptomatology. Additional clinical characteristics are presented in Table 2.

\section{Table 2}

\section{Clinical Characteristics}

\begin{tabular}{lll}
\hline Age at Diagnosis & Mean (SD) & $52.18(13.39)$ \\
\hline & Frequency & Percent \\
\hline Stage & & \\
0 (DCIS) & 5 & $8.5 \%$ \\
I & 25 & $42.4 \%$ \\
IIA & 7 & $11.9 \%$ \\
IIB & 4 & $6.8 \%$ \\
IIIA & 10 & $16.9 \%$ \\
IIIB & 1 & $1.7 \%$ \\
IIIC & 3 & $5.1 \%$ \\
IV & 4 & $6.8 \%$ \\
Grade & & \\
Well-differentiated & 8 & $13.6 \%$ \\
Moderately-differentiated & 23 & $39.0 \%$ \\
Poorly-differentiated & 20 & $33.9 \%$ \\
& & \\
\hline
\end{tabular}




\begin{tabular}{|c|c|c|}
\hline \multicolumn{3}{|l|}{ ER Status } \\
\hline Positive & 41 & $77.4 \%$ \\
\hline Negative & 12 & $22.6 \%$ \\
\hline \multicolumn{3}{|l|}{ PR Status } \\
\hline Positive & 39 & $75.0 \%$ \\
\hline Negative & 13 & $25.0 \%$ \\
\hline \multicolumn{3}{|l|}{ Her2Neu Status } \\
\hline Positive & 5 & $8.5 \%$ \\
\hline Negative & 42 & $89.4 \%$ \\
\hline \multicolumn{3}{|l|}{ Triple Negative } \\
\hline Yes & 8 & $17.0 \%$ \\
\hline No & 39 & $83.0 \%$ \\
\hline \multicolumn{3}{|l|}{ Lymphovascular Invasion } \\
\hline Yes & 12 & $25.5 \%$ \\
\hline No & 35 & 74.55 \\
\hline \multicolumn{3}{|c|}{ Primary, Recurrence, or Metastatic } \\
\hline Primary & 52 & $88.1 \%$ \\
\hline Recurrence or Metastatic & 7 & $11.9 \%$ \\
\hline \multicolumn{3}{|l|}{ Menopausal Status } \\
\hline Pre-menopausal & 23 & $40.4 \%$ \\
\hline Peri-menopausal & 4 & $7.0 \%$ \\
\hline Post-menopausal & 30 & $52.6 \%$ \\
\hline \multicolumn{3}{|l|}{ Karnofsky Rating } \\
\hline $100 \%$ & 23 & $44.2 \%$ \\
\hline $90 \%$ & 20 & $38.5 \%$ \\
\hline $80 \%$ & 6 & $11.5 \%$ \\
\hline $70 \%$ & 1 & $1.9 \%$ \\
\hline $60 \%$ & 2 & $3.8 \%$ \\
\hline
\end{tabular}

Co-morbid medical disorders. Three participants (5.1\%) reported having recurrent breast cancer and had previously received chemotherapy and/or radiation. Additionally, one participant (1.7\%) reported a history of previous cervical cancer. While the majority of participants reported no co-morbid medical conditions $(n=29 ; 49.1 \%)$, the most common co-morbid medical conditions for this sample were high blood pressure $(\mathrm{n}=15 ; 25.4 \%)$ and diabetes $(\mathrm{n}=10 ; 17.0 \%)$. Other co-morbid medical conditions included arthritis/osteoarthritis $(n=3 ; 5.1 \%)$, hypothyroidism $(n=2 ; 3.4 \%)$, and myocardial infarction $(n=2 ; 3.4 \%)$. One participant $(1.7 \%)$ reported having scoliosis and one $(1.7 \%)$ reported having chronic obstructive pulmonary disease. Additionally, one participant (1.7\%) reported having chronic fatigue syndrome, simple partial seizures, skin 
lesions, and chronic hives; one reported having scleroderma and local positive antinuclear antibody, which is a diagnostic test for lupus; one reported having supraventricular and ventricular tachycardia and a heart murmur, in addition to hypertension; and one reported sarcoidosis and asthma, in addition to hypertension and osteoarthritis.

Medications. Table 3 presents medications used by greater than $10 \%$ of participants. Additionally, less than $10 \%$ of participants used medications such as antihistaminics and sleep medications $(\mathrm{n}=5)$; analgesics, bronchodilators, thyroid medication, and vitamin supplements $(\mathrm{n}=4)$; anticonvulsants, antitussives, benzodiazepines, potassium, and urinary antispasmodics $(n=3)$; adrenergics, antibiotics, estrogens, and vasodilating agents $(\mathrm{n}=2)$, and anticoagulants, nasal anti-inflammatory agents, antidiarrheal medications, antirheumatoids, antiviral medications, asthma medications, cathartics and laxatives, contraceptives, corticosteroids, iron replacements, insulin, muscle relaxants, prophylaxis, proton pump inhibitor, parathyroid hormones, respiratory relaxer, sedatives, sulfonylureas, and unclassified therapeutics $(\mathrm{n}=1)$.

\section{Table 3}

\section{Medications}

\begin{tabular}{lll}
\hline & Frequency & Percent \\
\hline Antihypertensive & 16 & $27.1 \%$ \\
NSAIDS (e.g., aspirin, ibuprofen) & 16 & $27.1 \%$ \\
Diuretics & 11 & $18.6 \%$ \\
Antilipidemic & 11 & $18.6 \%$ \\
Antidiabetics & 10 & $16.9 \%$ \\
Antidepressants & 9 & $15.3 \%$ \\
Cardiac Medications & 7 & $11.9 \%$ \\
Anxiolytics & 6 & $10.2 \%$ \\
Gastrointestinal Medications & 6 & $10.2 \%$ \\
Opiate Agonists & 6 & $10.2 \%$ \\
\hline
\end{tabular}

\section{Psychosocial Stress Characteristics}


Ethnicity. Fifty-seven participants provided information regarding ethnicity. The majority of participants were non-Hispanic $(\mathrm{NH})$ White $(\mathrm{n}=33 ; 57.9 \%)$ and African American $(\mathrm{n}=21 ; 36.8 \%)$. Two participants reported their ethnicity as Native American (3.5\%), and one participant reported her ethnicity as Asian American (1.8\%). As previously reported, analyses excluded participants who reported an ethnicity other than $\mathrm{NH}$ White or African American out of respect for potential cultural/ethnic differences on outcome variables. Additionally, the small sample size would not allow statistical analyses to explore ethnic differences for those who reported their ethnicity as Native American or Asian American.

Neighborhood stress. Census tract data was available for 47 participants. Neighborhood stress was measured with four variables, the percentage of households below the poverty level within the census tract, the median household income in the census tract, the percentage of single-parent homes within the census tract, and the percentage of renter-occupied homes within the census tract. The 47 participants lived in a total of 41 different census tracts. Within the census tracts, the average (SD) percentage of households below the poverty level was 16.66 (12.71), the average (SD) median household income was $\$ 50,868.72$ ( $\$ 23,617.78)$, the average (SD) percentage of singleparent homes was 39.84 (24.82), and the average (SD) percentage of renter-occupied homes was $31.16(19.25)$.

Cancer-related stress. The Impact of Events Scale (IES) total distress score was used to measure participants' current amount of distress related to their cancer. Fifty-six participants provided IES data. During data cleaning, an error with the IES wording was identified. For the second half of our participants, the wording on the IES had been 
changed, such that the Likert scale read "not at all, rarely, sometimes, and never" rather than the appropriate "not at all, rarely, sometimes, and often." It was determined that one participant who answered endorsed a "3" truly meant "never" and did not mean "often." By viewing a scatterplot of IES baseline and IES daily comparison scores that one participant was an outlier. We then reviewed her IES baseline raw data with her IES intrusions daily raw data. It was determined that her baseline raw data did not match the daily raw data based on the IES daily scores of mostly zero. Based on this, we changed her "threes" to "zeros" on the IES baseline raw data to better match her apparent intended responses. Therefore, that participant's data was edited accordingly, and the total score was then calculated with this edited data.

The average (SD) total distress score was 28.38 (15.37), which suggests the presence of symptoms of both intrusive thoughts and avoidance of thoughts related to a cancer diagnosis. Scores of 19 or greater are worthy of additional investigation and scores 35 or greater likely represents a diagnosis of PTSD (Horowitz et al., 1979). In this sample, $43(76.8 \%)$ participants scored 19 or greater and $26(46.4 \%)$ participants scored 35 or greater on the IES.

\section{Physiological Disruption Characteristics}

Circadian disruption. Descriptive statistics for wrist-worn actigraphy variables are presented in Table 4. Of the 57 participants, one participant was provided with a watch that was initialized in the wrong recording mode, which meant that the data that was returned was unusable for the calculations. One participant's actiwatch battery failed and there was not time to collect replacement data before surgical treatment. Therefore, the resulting sample size for actigraphy data was 55 . The average (SD) rest/activity 
rhythm coefficient of $.28(.17)$ is indicative of a relatively rhythmic circadian activity pattern in this sample. Additionally, percentages for nighttime restfulness $(97.38 \%)$ and daytime sedentariness $(6.03 \%)$ show that many of the sample experienced restful evenings and a good amount of activity during the day, which are indicative of high circadian rhythmicity.

\section{Table 4}

Actigraphy Descriptive Statistics

\begin{tabular}{lll}
\hline & $\mathrm{N}$ & Mean (SD) \\
\hline Rest/activity rhythm & 55 & $.28(.17)$ \\
Nighttime restfulness, \%age & 55 & $97.38(3.26)$ \\
Daytime sedentariness, \%age & 55 & $6.03(5.41)$ \\
\hline
\end{tabular}

Endocrine disruption. Descriptive statistics for diurnal cortisol variables are presented in Table 5. Note that the CAR slope is more vulnerable to missing data than the other variables; therefore, the sample size for CAR slope is lower than the sample size for diurnal cortisol slope and diurnal cortisol mean. Average (SD) raw cortisol for the threeday collection was $0.25(.11)$. Average and 95\% confidence intervals for raw cortisol values for each point of data collection point are presented in Figure 8, which appears to resemble a healthy diurnal cortisol slope, with a peak approximately 30 minutes after awakening and a nadir at bedtime.

\section{Table 5}

Diurnal Cortisol Descriptive Statistics

\begin{tabular}{lll}
\hline & $\mathrm{N}$ & Mean $(\mathrm{SD})$ \\
\hline CAR Slope, $\log (\mu \mathrm{g} / \mathrm{dL}) / \mathrm{hr}$ & 45 & $.10(.47)$ \\
Diurnal Cortisol slope, $\log (\mu \mathrm{g} / \mathrm{dL}) / \mathrm{hr}$ & 57 & $-.07(.09)$ \\
Diurnal Mean Cortisol, $\log (\mu \mathrm{g} / \mathrm{dL})$ & 57 & $-1.89(.48)$ \\
\hline
\end{tabular}




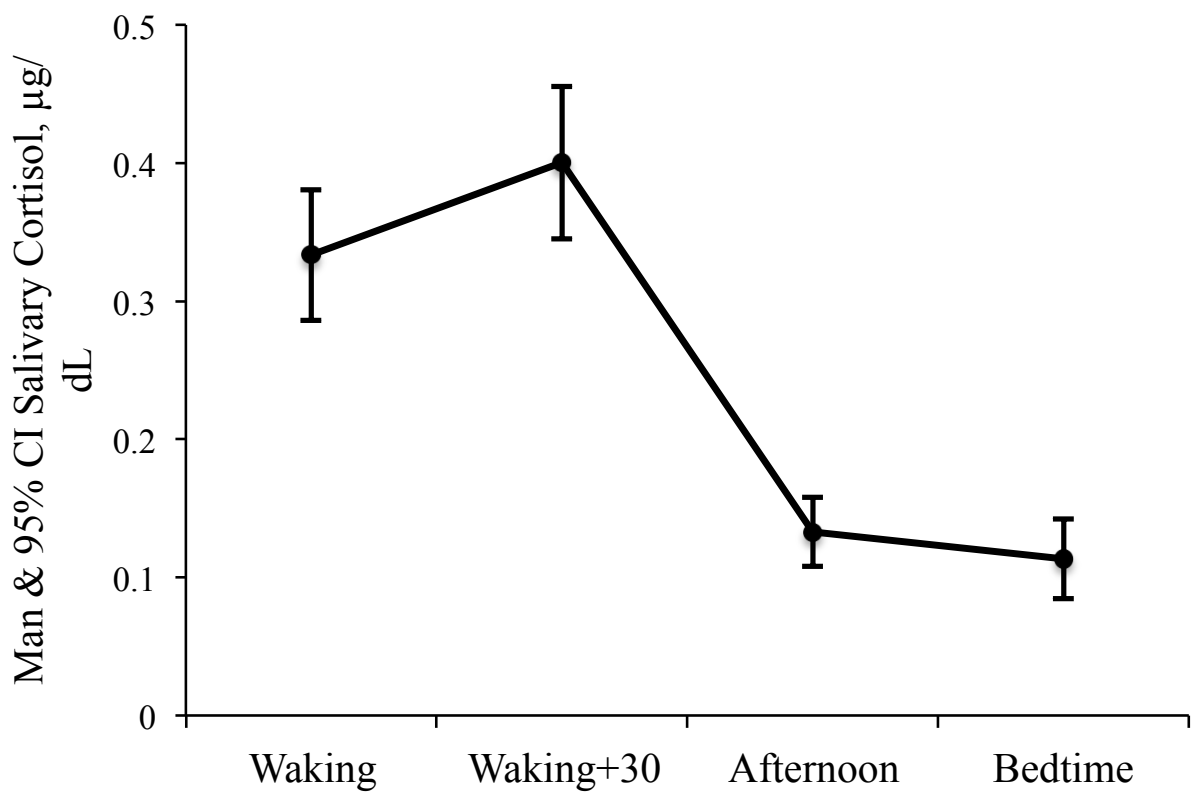

Figure 8 . Mean with $95 \%$ confidence interval diurnal salivary cortisol values over the three-day data collection period.

Immune function. Descriptive statistics for serum cytokine levels are provided in

Table 6. Serum cytokines were grouped into three factors using EFA (previously

described, Cash et al., 2015). Factor 1 includes VEGF, MMP-9, and TGF- $\beta$, factor 2

includes IL-1 $\beta$, IL-6R, TNF- $\alpha$, and MCP-1, and factor 3 includes IL-6, IL-12, and IFN- $\gamma$.

\section{Table 6}

Immune Function Descriptive Statistics

\begin{tabular}{lll}
\hline & $\mathrm{N}$ & Mean (SD) \\
\hline Factor 1 & & \\
VEGF, pg/mL & 50 & $383.99(228.86)$ \\
MMP-9, pg/mL & 50 & $28.84(18.23)$ \\
TGF- $\beta, \mathrm{pg} / \mathrm{mL}$ & 50 & $2.67(1.04)$ \\
Factor 2 & & \\
IL-1 $\beta, \mathrm{pg} / \mathrm{mL}$ & 50 & $0.52(2.05)$ \\
IL-6R, pg/mL & 50 & $6242.39(1687.03)$ \\
TNF- $\alpha, \mathrm{pg} / \mathrm{mL}$ & 50 & $24.30(115.03)$ \\
MCP-1, pg/mL & 50 & $148.19(60.00)$ \\
Factor 3 & & \\
IL-6, pg/mL & 50 & $19.19(120.41)$
\end{tabular}


IL-12, pg/mL $\quad 50 \quad 17.95(31.28)$

IFN- $\gamma, \mathrm{pg} / \mathrm{mL} \quad 50 \quad 1.16(1.07)$

\section{Progression-Free Survival}

Data on progression-free survival was obtained from KCR at a median of seven years post-enrollment and was available for 53 participants. Of the 53 participants, $30.2 \%$ $(\mathrm{n}=16)$ had experienced a recurrence or had passed away. Six women had recurrent disease, but had not passed away. Ten participants had passed away, of which eight had recurrent disease. Descriptive statistics for progression-free survival from date of diagnosis or date of data collection are provided in Table 7.

\section{Table 7}

Progression-Free Survival Descriptive Statistics

\begin{tabular}{|c|c|c|c|}
\hline & \multirow[b]{2}{*}{$\mathrm{N}$} & Days & Years \\
\hline & & \multicolumn{2}{|c|}{ Mean (SD) } \\
\hline From Date of Diagnosis & 49 & $2103.63(823.84)$ & $5.76(2.26)$ \\
\hline From Date of Background Completion & 49 & $2000.33(799.90)$ & $5.48(2.19)$ \\
\hline From Date of Actigraphy Collection & 49 & $2081.59(810.58)$ & $5.70(2.22)$ \\
\hline From Date of Cortisol Collection & 52 & $2030.46(795.48)$ & $5.56(2.18)$ \\
\hline From Date of Blood Draw & 45 & $1984.13(789.22)$ & $5.43(2.16)$ \\
\hline
\end{tabular}

\section{Principal Components Analysis of Neighborhood Stress}

A principal components analysis (PCA) was performed with orthogonal rotation (varimax) to determine the degree to which the four variables of neighborhood stress that were collected (i.e., percentage of households below the poverty line, median household income, percentage of single-parent homes, and percentage of renter-occupied housing in the neighborhood) varied together. Given that the data were skewed and on different scales (i.e., percentages and median values), the data were transformed and standardized to address each concern, respectively. 
The Kaiser-Meyer-Olkin (KMO) statistic, a measure of sampling adequacy, was .852 , which is in the "great" range and is indicative that the sample size is adequate and the PCA will yield distinct and reliable factors (Field, 2009). Additionally, all KMO values for individual items were greater than .82 , which is well above the acceptable limit of .5 (Field, 2009). Bartlett's test of sphericity indicated that correlations between items were sufficiently large enough to run PCA, $\chi^{2}(6)=166.56, p<.001$ (Field, 2009). One variable had an eigenvalue greater than one, and through examination of the scree plot, there was one point of inflection, which justify that the four variables in the PCA cluster to form one factor (Field, 2009), which represents neighborhood stress. Factor scores for each participant were obtained using the regression method in SPSS, and these scores were used as the independent variable in analyses. Table 8 provides the anti-image correlation matrix for the PCA. Table 9 provides the eigenvalues and total variance explained by the PCA. Table 10 provides the factor loadings.

\section{Table 8}

\section{Anti-Image Correlation Matrix}

\begin{tabular}{|c|c|c|c|c|}
\hline & $\begin{array}{l}\text { \% Households } \\
\text { Below Poverty } \\
\text { Level }\end{array}$ & $\begin{array}{l}\text { Median } \\
\text { Household } \\
\text { Income } \\
\end{array}$ & $\begin{array}{l}\text { \% Single- } \\
\text { Parent Homes }\end{array}$ & $\begin{array}{l}\text { \% Renter- } \\
\text { Occupied } \\
\text { Homes }\end{array}$ \\
\hline $\begin{array}{l}\text { \% Households } \\
\text { Below Poverty } \\
\text { Level }\end{array}$ & $.828 *$ & .503 & -.136 & -.384 \\
\hline $\begin{array}{l}\text { Median } \\
\text { Household } \\
\text { Income }\end{array}$ & -- & $.832 *$ & .372 & .121 \\
\hline $\begin{array}{l}\% \text { Single-Parent } \\
\text { Homes }\end{array}$ & -- & -- & $.878^{*}$ & -.330 \\
\hline $\begin{array}{l}\% \text { Renter- } \\
\text { Occupied } \\
\text { Homes }\end{array}$ & -- & -- & -- & $.875^{*}$ \\
\hline
\end{tabular}

* Measures of Sampling Adequacy 


\section{Table 9}

$\underline{\text { Total Variance Explained }}$

\begin{tabular}{|c|c|c|c|c|c|c|}
\hline \multirow[b]{2}{*}{ Component } & \multicolumn{3}{|c|}{ Initial Eigenvalues } & \multicolumn{3}{|c|}{$\begin{array}{c}\text { Extraction Sums of Squared } \\
\text { Loadings }\end{array}$} \\
\hline & Total & $\begin{array}{c}\% \text { of } \\
\text { Variance }\end{array}$ & $\begin{array}{c}\text { Cumulative } \\
\%\end{array}$ & Total & $\begin{array}{c}\% \text { of } \\
\text { Variance }\end{array}$ & $\begin{array}{c}\text { Cumulative } \\
\%\end{array}$ \\
\hline $\begin{array}{l}\text { \% Households } \\
\text { Below Poverty } \\
\text { Level }\end{array}$ & 3.423 & 85.576 & 85.576 & 3.423 & 85.576 & 85.576 \\
\hline $\begin{array}{l}\text { Median Household } \\
\text { Income }\end{array}$ & 0.225 & 5.614 & 91.190 & & & \\
\hline $\begin{array}{l}\text { \% Single-Parent } \\
\text { Homes }\end{array}$ & 0.220 & 5.504 & 96.694 & & & \\
\hline $\begin{array}{l}\% \text { Renter- } \\
\text { Occupied Homes }\end{array}$ & 0.132 & 3.306 & 100.000 & & & \\
\hline
\end{tabular}

Table 10

Factor Loadings

\begin{tabular}{lll}
\hline Factor & Variables Included & Factor Loading \\
\hline 1 & \% Households Below Poverty Level & .936 \\
& Median Household Income & -.934 \\
& \% Single-Parent Homes & .915 \\
& \% Renter-Occupied Homes & .915 \\
\hline
\end{tabular}

\section{Primary Analyses}

For tests of hypotheses, significant results are presented in tables. When multiple independent variables were entered to test the same outcome (e.g., survival analyses with three actigraphy measures all relating to survival), data are presented from step 1 of only one model. Effect sizes for each step of the model are denoted by $R^{2}$ and $\Delta R^{2}$ values presented in each table. Full result tables are presented in Appendix C.

Hypothesis 1A, 1B, and 1C. Ethnicity, neighborhood stress, and cancerrelated stress will be significantly and positively correlated with one another (Figure 
2). Ethnicity and neighborhood stress were highly correlated, $\mathrm{r}(43)=.615, p<.001$. NH White ethnicity was statistically significantly correlated with lower neighborhood stress, whereas African American ethnicity was statistically significantly correlated with greater neighborhood stress. Neither ethnicity nor neighborhood stress was significantly correlated with cancer-related stress. The correlation matrix is presented in Table 11.

\section{Table 11}

Spearman's rho correlation matrix for hypothesis 1.

\begin{tabular}{llll}
\hline & 1 & 2 & 3 \\
\hline 1. Ethnicity & -- & $.615^{*}$ & -.066 \\
2. Neighborhood & -- & -- & .172 \\
stress & & -- & -- \\
$\begin{array}{l}\text { 3. Cancer-related } \\
\text { stress }\end{array}$ & -- & & \\
\hline$* p<.001$ & & &
\end{tabular}

Hypothesis 2. Tests of hypotheses 2 will explore associations of psychosocial stress (i.e., ethnicity, neighborhood stress, and cancer-related distress) with physiological disruption (i.e., circadian, endocrine, and immune; Figure 1, Arrow A), associations of psychosocial stress and medical outcomes of cancer (i.e., progression-free survival; Figure 1, Arrow B), and associations of physiological stress and medical outcomes of cancer (Figure 1, Arrow C).

Ethnicity as a predictor. Hypothesis 2A.1. African American ethnicity will be independently associated with greater circadian disruption, endocrine disruption, and immune disruption.

Circadian outcomes (Figure 3). In the model of ethnicity predicting rest/activity rhythms, control variables as a group $\left(\Delta \mathrm{R}^{2}=.223 ; \mathrm{F}(3,39)=3.728, p=.019\right)$ were significant predictors in the model and income added significant predictive power to the 
model (partial $r=.442$; Figure 9). The overall model was not significant. Patients with lower income had less rhythmic circadian patterns of activity.

In the model of ethnicity predicting nighttime restfulness, control variables were not significant predictors of nighttime restfulness as a group, nor were they significant individually. The overall model was not significant.

Control variables as a group $\left(\Delta \mathrm{R}^{2}=.185 ; \mathrm{F}(3,39)=2.942, p=.045\right)$ were significant predictors in the model of ethnicity predicting daytime sedentariness, and income added significant predictive power to the model (partial $r=-.378$; Figure 9). The overall model was not significant. Patients with lower income had greater circadian disruption.

Endocrine outcomes (Figure 3). In the models of ethnicity predicting endocrine disruption, control variables were not significant as a group, nor were they significant individually. Additionally, ethnicity did not emerge as significantly associated with variables of endocrine disruption.

Immune outcomes (Figure 3). In both the model of ethnicity predicting immune factor 1 and the model predicting immune factor 2, control variables were not significant as a group, nor were they significant individually. The overall models for factor 1 and factor 2 were not significant.

In the model of ethnicity predicting immune factor 3 (IL-6; IL-12; IFN- $\gamma$ ), control variables as a group $\left(\Delta \mathrm{R}^{2}=.342 ; \mathrm{F}(3,32)=5.546, p=.004\right)$ were significant predictors. Additionally, age added significant predictive power to the model (partial $r=-.566$; Figure 9). The overall model was not significant. Younger patients had greater secretion of inflammatory/ $\mathrm{T}_{\mathrm{H}} 1$-type cytokines (Factor 3: IL-6; IL-12; IFN- $\gamma$ ). 


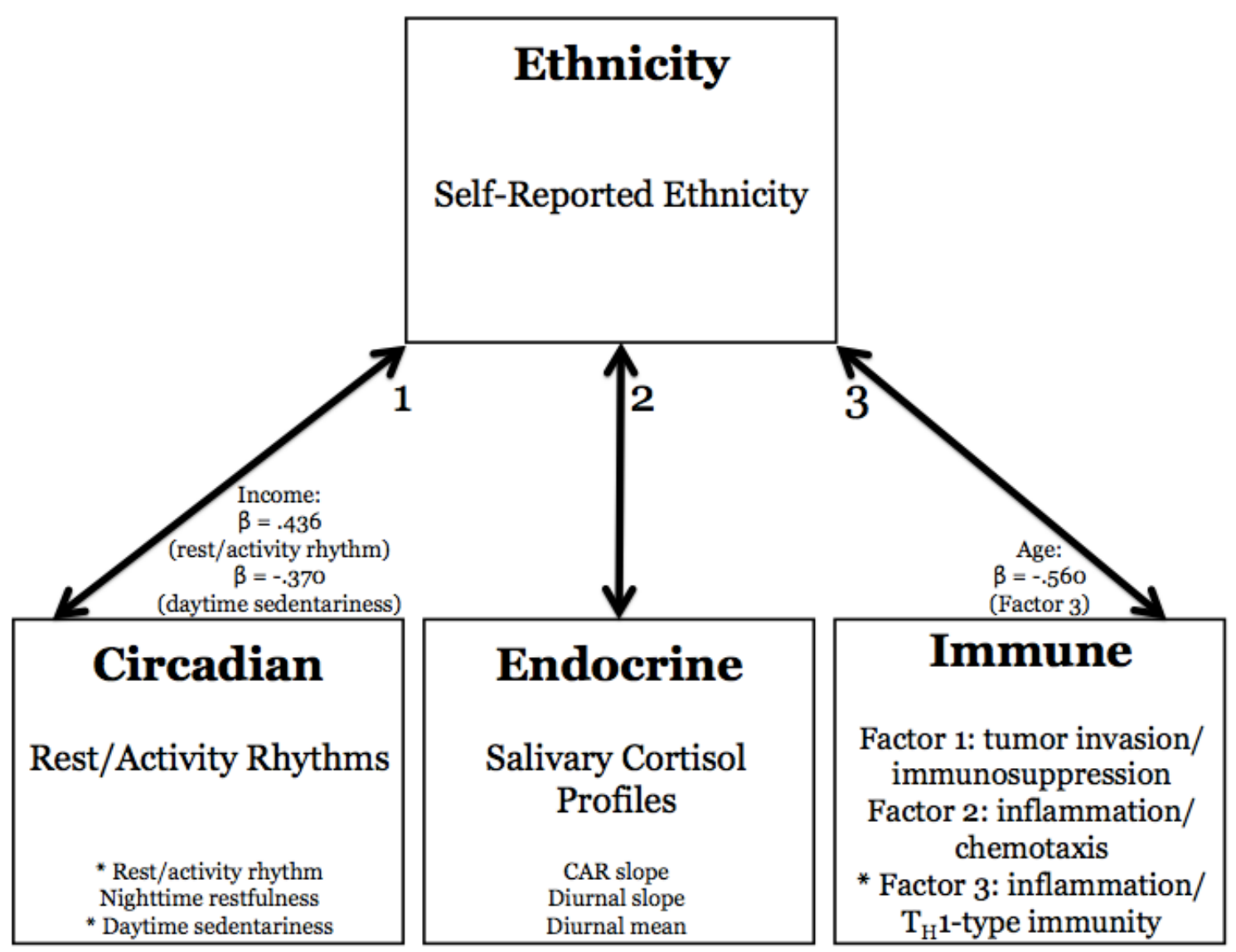

Figure 9. Significant associations with control variables for associations of ethnicity with physiological disruption.

Neighborhood stress as a predictor. Hypothesis 2A.2. Neighborhood stress will be independently associated with greater circadian disruption, endocrine disruption, and immune disruption.

Circadian outcomes (Figure 4). Control variables as a group $\left(\Delta \mathrm{R}^{2}=.281 ; \mathrm{F}(3,36)\right.$ $=4.680, p=.007)$ were significant predictors in the model neighborhood stress predicting rest/activity rhythms and income added significant predictive power (partial $\mathrm{r}-.503$;

Figure 11). However, the overall model was not significant. Patients with lower income had less rhythmic circadian pattern of activity. 
In the model of neighborhood stress predicting nighttime restfulness, control variables were not significant as a group, nor were they significant individually. The overall model was not significant

In the model of neighborhood stress predicting daytime sedentariness, control variables as a group were not significant. Income added significant predictive power to the model in predicting daytime sedentariness (partial $r=-.361$; Figure 11). The overall model was not significant. Patients with lower income had greater circadian disruption.

Endocrine outcomes (Figure 4). Control variables were not significant as a group, nor were they significant individually in the model of neighborhood stress predicting CAR slope. The overall model was not significant.

In the model testing relationships of control variables and neighborhood stress with diurnal cortisol slope, control variables were not significant as a group. However, income added significant predictive power to the model (partial $r=-.365$; Figure 11). The overall model was not significant. Patients with lower income had flatter slopes, which are indicative of a less healthy pattern.

The overall model of the association of neighborhood stress with diurnal cortisol mean was significant $\left(\Delta \mathrm{R}^{2}=.246 ; \mathrm{F}(4,36)=4.557, p=.001\right.$; Figure 10); however, control variables as a group were not significant, nor were they significant individually. Neighborhood stress was noted to be a significant predictor in the model testing diurnal cortisol mean (partial $r=.520$ ), demonstrating that patients with greater neighborhood stress had greater overall cortisol secretion, which is indicative of a less healthy pattern of cortisol secretion. Significant results are presented in Table 12. 


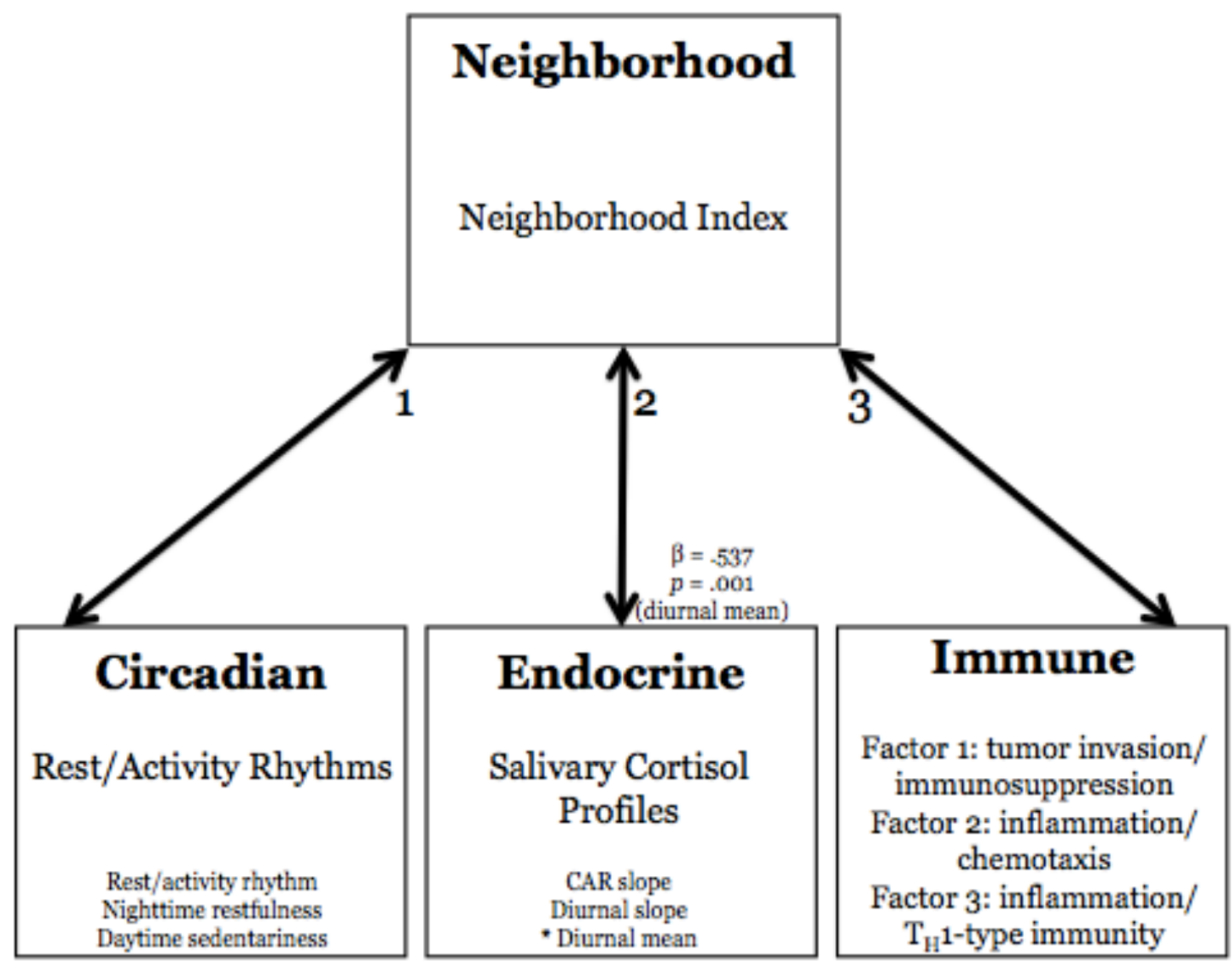

Figure 10. Significant association between neighborhood stress and endocrine disruption (i.e., diurnal mean).

Table 12

$\underline{\text { Significant results for hypothesis 2A.2: Hierarchical regression analyses entered }}$ neighborhood stress as a predictor of endocrine disruption.

\begin{tabular}{|c|c|c|c|c|c|c|c|}
\hline Variable & $\mathrm{N}$ & $\mathrm{B}$ & SE B & $\beta$ & $\mathrm{R}^{2}$ & $\Delta \mathrm{R}^{2}$ & $\mathrm{p}$ of $\Delta \mathrm{R}^{2}$ \\
\hline Diurnal cortisol mean & 41 & & & & & & \\
\hline Step 1 & & & & & .090 & .090 & .314 \\
\hline Age & & .011 & .006 & .277 & & & \\
\hline Stage & & .027 & .038 & .113 & & & \\
\hline Income & & -.022 & .046 & -.076 & & & \\
\hline Step 2 & & & & & .336 & .246 & $.001 *$ \\
\hline Neighborhood stress & & .290 & .079 & .537 & & & \\
\hline
\end{tabular}

$* p<.05$ 
Immune outcomes (Figure 4). In both the model of neighborhood stress predicting immune factor 1 and the model predicting immune factor 2, control variables were not significant as a group, nor were any of them significant individually. The overall models for factor 1 and factor 2 were not significant.

In the model of neighborhood stress predicting immune factor 3 (IL-6; IL-12; IFN- $\gamma)$, control variables as a group $\left(\Delta \mathrm{R}^{2}=.438 ; \mathrm{F}(3,30)=7.799, p=.001\right)$ were significant predictors in the model. Additionally, age (partial $r=-.622$; Figure 10) and stage (partial $r=-.396$; Figure 11) added significant predictive power to the model predicting immune factor 3 (IL-6; IL-12; IFN- $\gamma$ ). However, the overall model was not significant. Younger patients and patients who had lower stage disease had greater section of inflammatory/ $\mathrm{T}_{\mathrm{H}} 1$-type cytokines (Factor 3: IL-6; IL-12; IFN- $\gamma$ ).

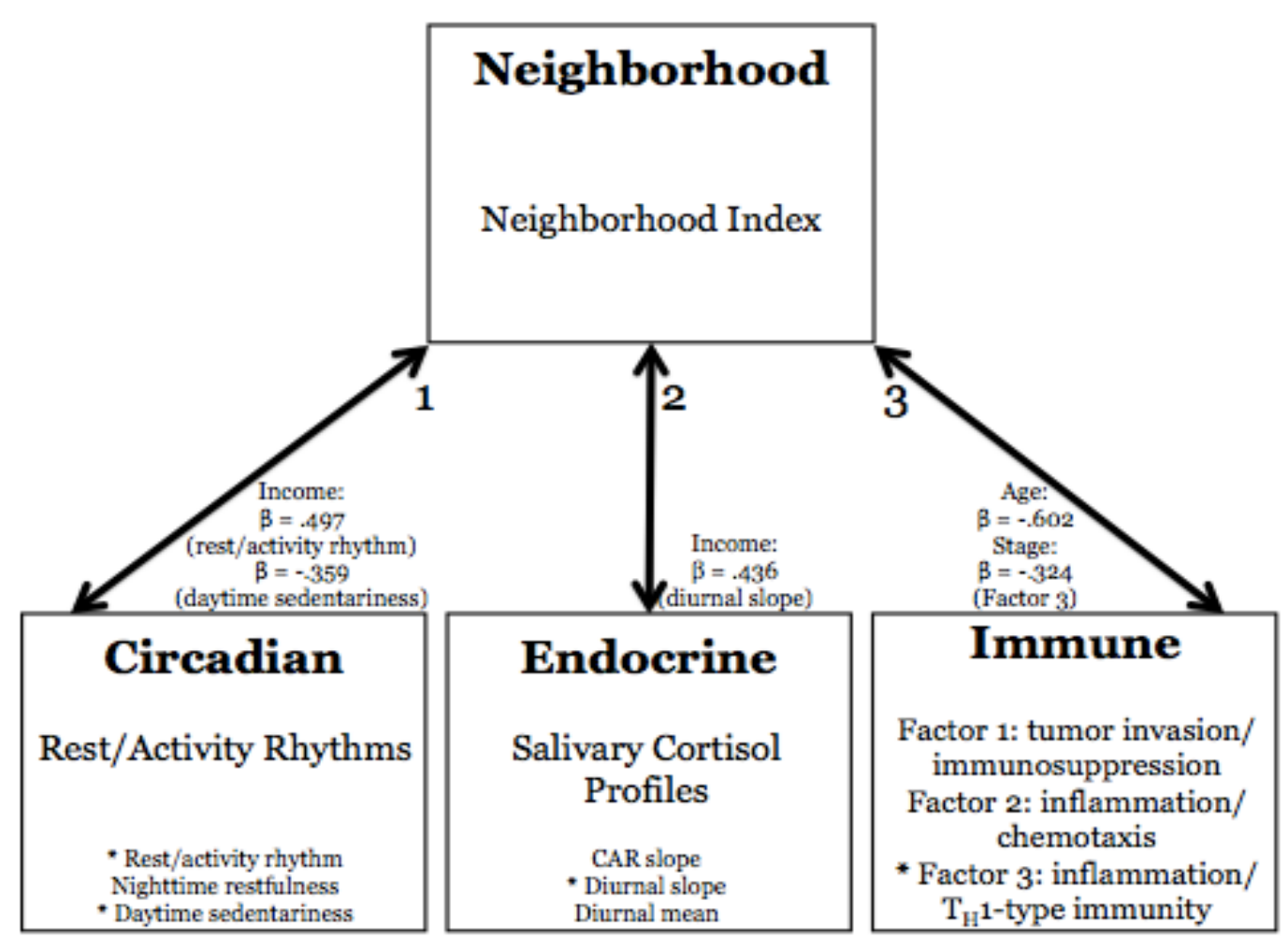

Figure 11. Significant associations with control variables for associations of neighborhood stress with physiological disruption. 
Cancer-related stress as a predictor. Hypothesis 2A.3. Cancer-related stress will be independently associated with greater circadian disruption, endocrine disruption, and immune disruption.

Circadian outcomes (Figure 5). Control variables as a group $\left(\Delta \mathrm{R}^{2}=.236 ; \mathrm{F}(3,41)\right.$ $=4.225, p=.011)$ were significant predictors in the model of cancer-related stress predicting rest/activity rhythms. Income added significant predictive power to the model in in predicting rest/activity rhythms (partial $r=.465$; Figure 14 ). The overall model was not significant. Patients with lower income had less rhythmic circadian pattern of activity. In the model predicting nighttime restfulness, control variables were not significant as a group, nor were they significant individually. The overall model was also not significant.

In the model predicting daytime sedentariness, control variables as a group were not significant; however, income added significant predictive power to the model (partial $r=-.370$; Figure 14). The overall model was not significant. Patients with lower income had greater circadian.

Endocrine outcomes (Figure 5). In the model of cancer-related stress predicting CAR slope, control variables were not significant as a group, nor were they significant individually. Additionally, the overall model was not significant.

The association of cancer-related stress with diurnal cortisol slope was significant in the overall model $\left(\Delta \mathrm{R}^{2}=.084 ; \mathrm{F}(1,42)=4.182, p=.047\right.$; Figure 12). Control variables were not significant as a group, nor were they significant individually. Cancer-related stress was noted to be a significant predictor in the model testing diurnal cortisol slope (partial $r=-.301$; Figure 12), demonstrating that patients with greater cancer-related 
stress had steeper slopes, which is indicative of a healthier pattern. For descriptive purposes, mean and $95 \%$ confidence interval raw cortisol values are shown across three days of sample collection. IES summary scores were split at median IES (Figure 13).

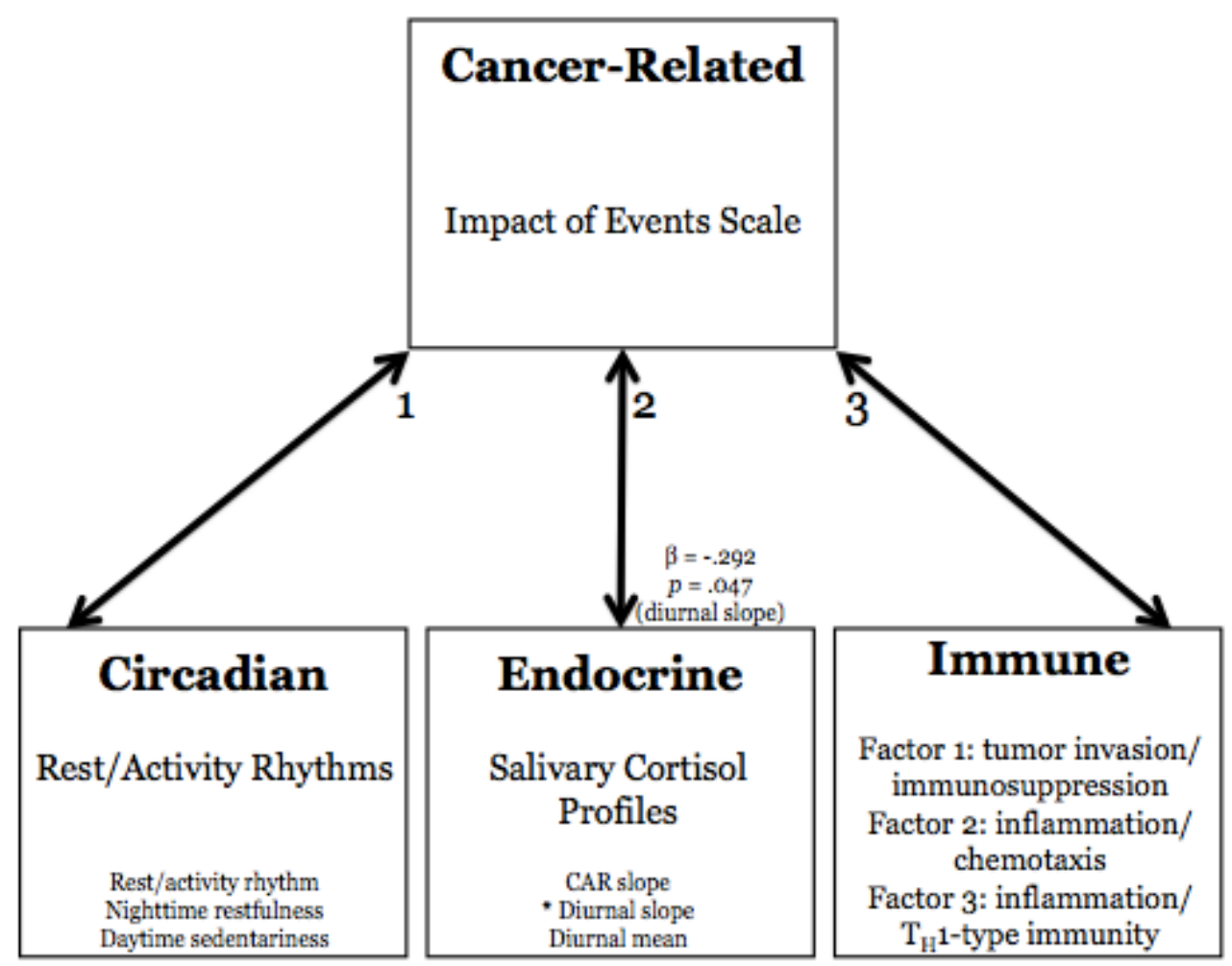

Figure 12. Significant association between cancer-related stress and endocrine disruption (i.e., diurnal slope). 


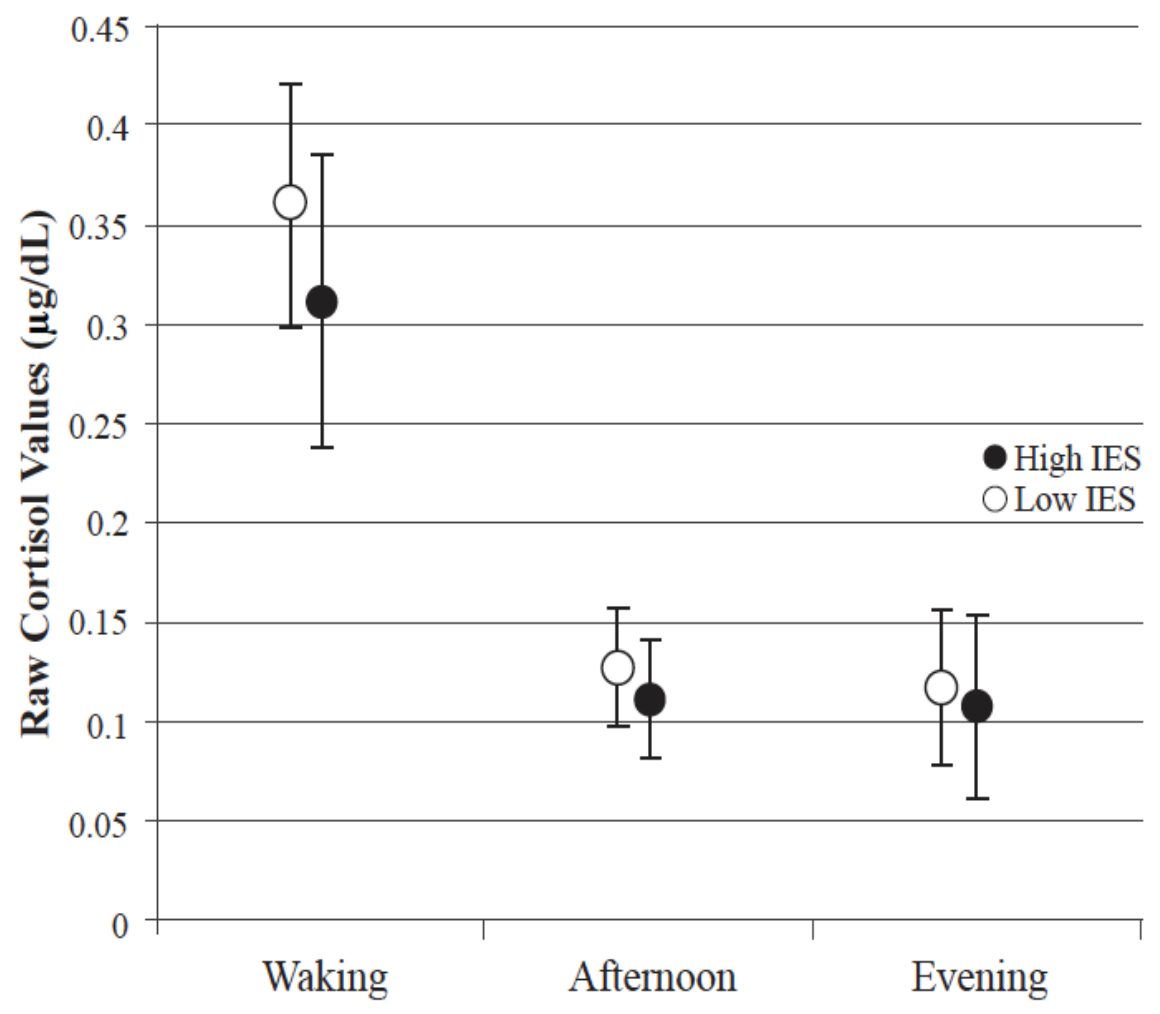

Figure 13. Mean and 95\% confidence interval raw diurnal cortisol values with median split IES summary scores.

In the model of cancer-related stress predicting diurnal cortisol mean, control variables were not significant as a group, nor were they significant individually. The overall model was not significant. Significant results are presented in Table 13.

\section{Table 13}

Summary of results for hypothesis 2A.3: Hierarchical regression analyses entered cancerrelated stress as a predictor of endocrine disruption.

\begin{tabular}{lccccccr}
\hline Variable & $\mathrm{N}$ & $\mathrm{B}$ & $\mathrm{SE} \mathrm{B}$ & $\beta$ & $\mathrm{R}^{2}$ & $\Delta \mathrm{R}^{2}$ & $\mathrm{p}$ of $\Delta \mathrm{R}^{2}$ \\
\hline Diurnal cortisol slope & 47 & & & & & & \\
Step 1 & & & & & .071 & .071 & .358 \\
Age & & -.001 & .001 & -.099 & & & \\
Stage & -.002 & .006 & -.053 & & & \\
Income & -.013 & .008 & -.258 & & & \\
Step 2 & & & & & .156 & .084 & $.047^{*}$ \\
Cancer-related stress & & -.002 & .001 & -.292 & & & \\
\hline
\end{tabular}

$* p<.05$ 
Immune outcomes (Figure 5). In both the model of cancer-related stress predicting immune factor 1 and the model predicting immune factor 2, control variables were not significant as a group, nor were they significant individually. The overall models for factor 1 and factor 2 were not significant.

Control variables as a group $\left(\Delta \mathrm{R}^{2}=.288 ; \mathrm{F}(3,35)=4.170, p=.007\right)$ were significant predictors in the model of cancer-related stress predicting immune factor 3 (IL-6; IL-12; IFN- $\gamma$ ). Additionally, age (partial $r=$-.509; Figure 14) added significant predictive power to the model. However, the overall model was not significant. Younger patients had greater secretion of inflammatory/ $\mathrm{T}_{\mathrm{H}} 1$-type cytokines (Factor 3: IL-6; IL-12; IFN- $\gamma)$.

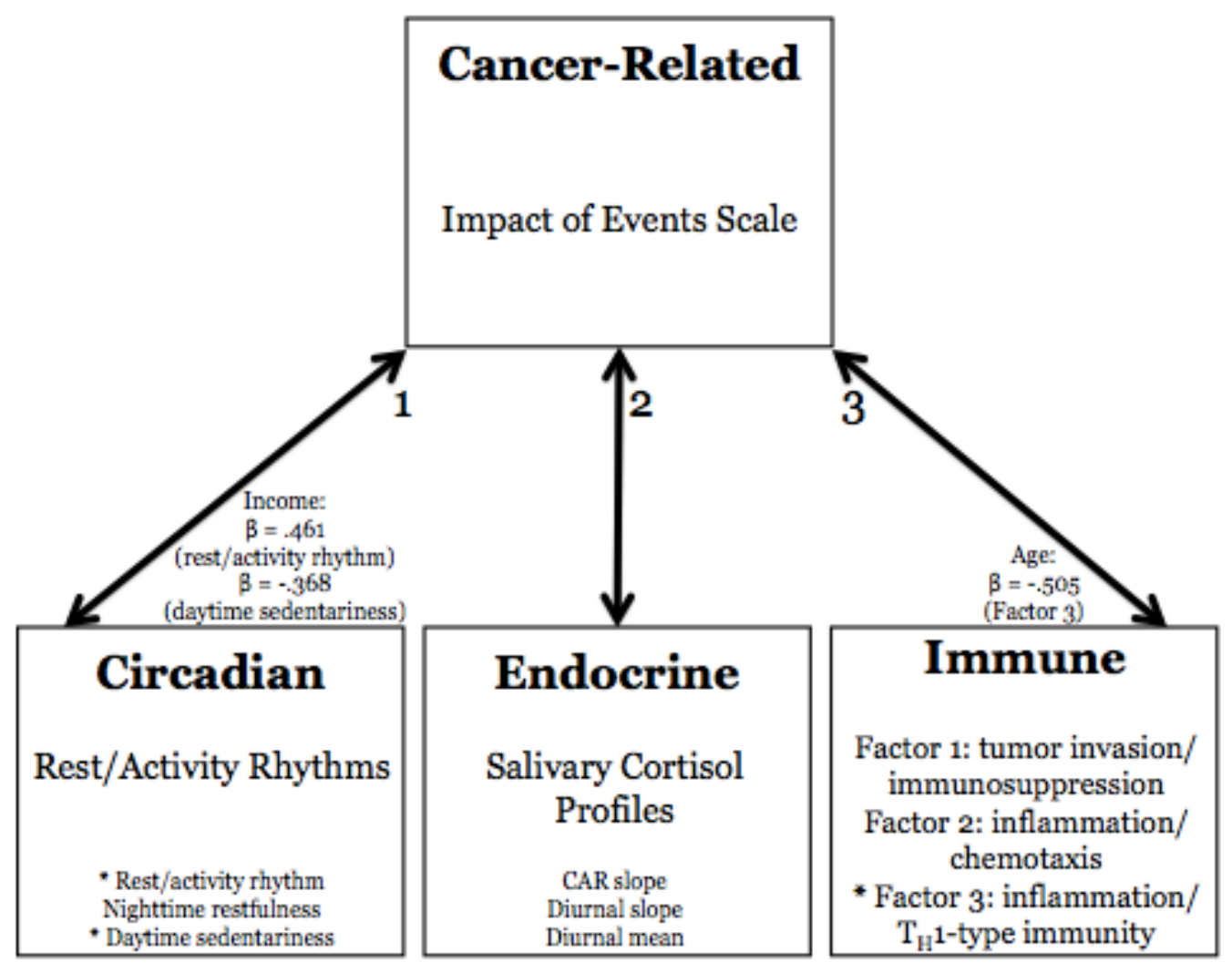

Figure 14. Significant associations with control variables for associations of cancer-related stress with physiological disruption. 


\section{Ethnicity as a predictor of progression-free survival (Figure 6, Arrow 1).}

Hypothesis 2B1. African American ethnicity will be independently associated with shorter breast cancer progression-free survival.

Ethnicity did not emerge as a significant predictor of progression-free survival measured from either date of diagnosis or date of study entry.

Neighborhood socioeconomic stress as a predictor of progression-free survival (Figure 6, Arrow 2). Hypothesis 2B.2. Neighborhood stress will be independently associated with shorter breast cancer progression-free survival.

Neighborhood stress did not emerge as a significant predictor of progression-free survival measured from either date of diagnosis or date of study entry.

\section{Cancer-related stress as a predictor of progression-free survival (Figure 6,}

Arrow 3). Hypothesis 2B.3. Cancer-related stress will be independently associated with shorter breast cancer progression-free survival.

Cancer-related stress did not emerge as a significant predictor of progression-free survival from either date of diagnosis or date of stress assessment.

\section{Circadian disruption variables as predictors of progression-free survival}

(Figure 7, Arrow 1). Hypothesis 2C.1. Circadian disruption will be independently associated with shorter breast cancer progression-free survival.

No circadian disruption variables (i.e., rest/activity rhythms, nighttime restfulness, and daytime sedentariness) emerged as significant predictors of progression-free survival from either date of diagnosis or date of first actigraphy assessment. 


\section{Endocrine disruption variables as predictors of progression-free survival}

(Figure 7, Arrow 2). Hypothesis 2C.2. Endocrine disruption will be independently associated with shorter breast cancer progression-free survival.

No endocrine disruption variables (i.e., CAR slope, diurnal cortisol slope, and diurnal cortisol mean) emerged as significant predictors of progression-free survival from either date of diagnosis or date of first cortisol collection.

Immune disruption variables as predictors of progression-free survival (Figure

7, Arrow 3). Hypothesis 2C.3. Immune disruption will be independently associated with shorter breast cancer progression-free survival.

No immune factors (i.e., Factor 1, Factor 2, and Factor 3) emerged as significant predictors of progression-free survival from either date of diagnosis or date of blood draw.

\section{Post hoc analyses.}

In tests of hypothesis $2 \mathrm{~A} .3$, cancer-related stress was related to diurnal cortisol slope, such that participants who endorsed greater cancer-related stress had steeper cortisol slopes, which are indicative of a healthy cortisol pattern. Given that these findings are in the opposite direction than hypothesized, post hoc analyses were conducted to gain a better understanding of this relationship. Upon examining subscales of the IES, the measure of cancer-related stress, avoidance (partial $r=-.410$ ), but not intrusions were significantly related to diurnal cortisol slope. For descriptive purposes, mean and $95 \%$ confidence interval raw cortisol values are shown across 3 days of sample collection. IES avoidance scores were split at the median (Figure 15). Therefore, patients 
who endorsed greater levels of cancer-related avoidance had steeper, healthier diurnal cortisol slopes. Post hoc analyses results are presented in Table 14.

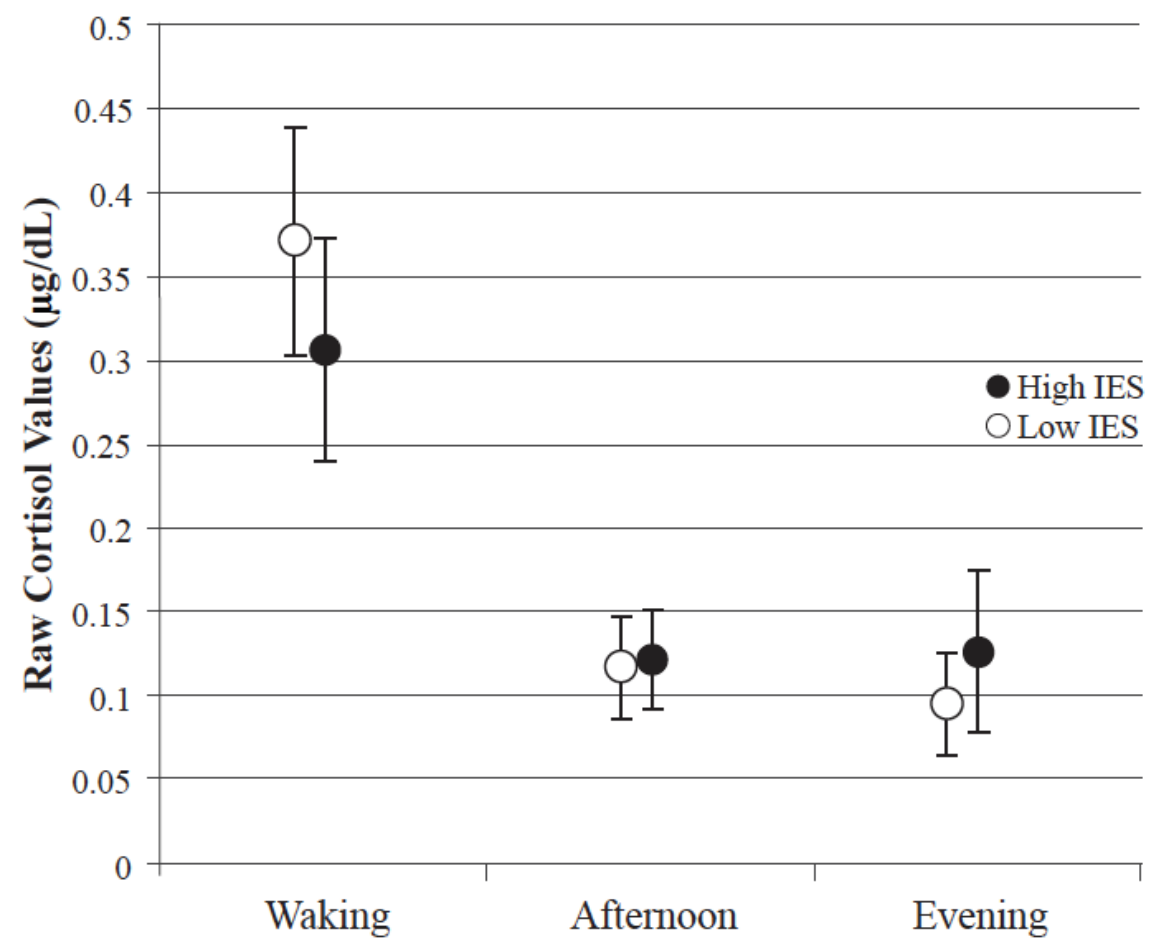

Figure 15. Mean and 95\% confidence interval raw diurnal cortisol values with median split IES avoidance scores.

\section{Table 14}

Summary of results for post hoc analyses: Hierarchical regression analyses entered cancer-related stress subscales as a predictor of diurnal cortisol slope.

\begin{tabular}{|c|c|c|c|c|c|c|c|}
\hline Variable & $\mathrm{N}$ & B & SE B & $\beta$ & $\mathrm{R}^{2}$ & $\Delta \mathrm{R}^{2}$ & $\mathrm{p}$ of $\Delta \mathrm{R}^{2}$ \\
\hline Diurnal Cortisol Slope & 48 & & & & & & \\
\hline Step 1 & & & & & .091 & .091 & 234 \\
\hline Age & & -.001 & .001 & -.124 & & & \\
\hline Stage & & -.003 & .006 & -.077 & & & \\
\hline Income & & -.015 & .008 & -.283 & & & \\
\hline Step 2 & & & & & .244 & .153 & $.005 *$ \\
\hline $\begin{array}{l}\text { Cancer-related stress: } \\
\text { Avoidance }\end{array}$ & & -.004 & .001 & -.399 & & & \\
\hline
\end{tabular}




\section{Summary of Results}

Primary analyses showed that ethnicity and neighborhood stress were statistically significantly associated, such that NH White patients had lower neighborhood stress and African American patients had greater neighborhood stress, which includes the percentage of households below the poverty line, median household income, percentage of single-parent homes, and percentage of renter-occupied housing in the neighborhood. This supports the hypothesis that ethnicity and neighborhood stress would be correlated, as both can be viewed measures of psychosocial stress. However, cancer-related stress was not significantly correlated with either ethnicity or neighborhood stress.

For associations of ethnicity with circadian variables, the control variables (i.e., age, stage, and income) as a group were significant predictors of rest/activity rhythms ( $p$ $=.019)$ and daytime sedentariness $(p=.045)$, with effect sizes of .223 and .185 , respectively. Control variables as a group were not significant predictors for associations of ethnicity with endocrine variables. For associations of ethnicity with immune variables, the control variables (i.e., age, stage, and income) as a group were significant predictors of Factor $3(p=.004)$ with an effect size of .342 .

For associations of neighborhood stress with circadian variables, the control variables (i.e., age, stage, and income) as a group were significant predictors of rest/activity rhythms $(p=.007)$ with an effect size of .281 . In analyses of relationships between neighborhood stress and endocrine disruption, control variables as a group were not significant predictors. Control variables as a group were significant predictors of Factor $3(p=.001)$ with an effect size of .438 in analyses of associations between neighborhood stress and immune disruption. Additionally, neighborhood stress was 
statistically significantly related to diurnal cortisol mean, such that patients with greater neighborhood stress had greater overall cortisol secretion, which is indicative of a less healthy pattern of cortisol secretion.

For associations of cancer-related stress with circadian variables, the control variables (i.e., age, stage, and income) as a group were significant predictors of rest/activity rhythms $(p=.011)$ with an effect size of .236; however, the control variables as a group were not significant predictors for associations of cancer-related stress with endocrine variables. In analyses testing associations of cancer-related stress with immune variables, control variables as a group were significant predictors of Factor $3(p=.007)$ with an effect size of .288. Cancer-related stress was statistically significantly related to diurnal cortisol slope, such that those reporting greater cancer-related stress had steeper diurnal cortisol slopes, which is indicative of a healthier cortisol profile. Post hoc analyses revealed that symptoms of avoidance, but not intrusions, were driving the relationships between cancer-related stress and diurnal cortisol slope, such that patients who endorsed greater avoidance had steeper slopes.

Analyses failed to support hypotheses that psychosocial stress factors are associated with variables of circadian disruption and immune disruption. However, two results were not statistically significant, but are worthy of discussion. The relationship between ethnicity and immune factor 2 (IL-1 $\beta$, IL-6R, TNF- $\alpha$, MCP-1) was not significant $(\mathrm{p}=.085)$, but the direction of the relationship indicates African American participants had lower levels of factor 1 immune parameters than NH White participants. The relationship between neighborhood stress and immune factor 1 (VEGF, MMP-9, TFG- $\beta$ ) was not significant $(p=.066)$; however, the relationship between the variables 
indicates that participants with greater neighborhood stress had lower levels of immune factor 1 parameters.

Interestingly, income was a significant control variable for the circadian disruption variables rest/activity rhythms and daytime sedentariness. Participants with lower income had less rhythmic circadian patterns of activity and greater daytime sedentary behavior, both of which are indicative of circadian disruption. Additionally, age was a significant control variable for immune factor 3 (IL-6, IL-12, IFN- $\gamma$ ). Younger participants had greater secretion of these immune parameters. In analyses exploring the relationship between neighborhood stress and diurnal cortisol slope, income arose as a significant control variable, such that participants with lower income had flatter cortisol slopes, indicative of a less healthy cortisol pattern.

Analyses failed to support the hypotheses that psychosocial stress factors and physiological disruption would be predictive of progression-free survival. 


\section{DISCUSSION}

This exploratory study examined the effects of psychosocial stress on physiological disruption in presurgical breast cancer patients. Additionally, the predictive value of psychosocial stress and physiological disruption on progression-free survival was also explored. As expected, ethnicity and neighborhood stress were correlated, such that African American participants had greater neighborhood stress. Therefore, it appears that the African American women in our sample are more likely to live in a neighborhood that can be viewed as socioeconomically stressful (i.e., a higher percentage of households below the poverty line, lower median household income, higher percentage of singleparent homes, and higher percentage of renter-occupied housing). Associations of ethnicity and neighborhood stress with cancer-related stress did not emerge.

Ethnicity was not related to any measure of physiological disruption (i.e., circadian disruption, endocrine disruption, and immune disruption). Although no significant associations were observed in these analyses, these associations should continue to be explored within a cancer context, as it is possible that physiological disruption may help to explain ethnic cancer disparities.

Participants experiencing greater neighborhood stress had greater overall diurnal cortisol mean secretion. This suggests that stress associated with the socioeconomic 
status of one's neighborhood may have an adverse impact on physiology. It is possible that the likely chronic nature of neighborhood stress alters physiology, specifically cortisol secretion. Additional analyses are warranted to explore how neighborhood stress impacts physiological disruption, particularly measures of chronic stress. Neighborhood stress was not associated with any other measure of physiological disruption.

Women experiencing greater cancer-related stress in our sample had steeper diurnal cortisol slopes, which can be indicative of a healthier cortisol profile. This finding is interesting in that it is in the opposite direction than anticipated. Post hoc analyses showed that avoidance, but not intrusions, were significantly associated with diurnal cortisol slope. Patients who endorsed greater avoidance had steeper slopes, which may indicate that during the presurgical period, avoidance may be a healthy way of coping through this acutely stressful period. Therefore, it is possible that in this sample of breast cancer patients who are presurgical, avoidance is serving as a buffer to stress, rather than a detriment, which may lead to a healthier diurnal cortisol pattern. Continued analysis is needed to determine the role of cancer-related stress, particularly avoidance, on physiological disruption during the presurgical phase of breast cancer treatment.

Cancer-related stress was not associated with measures of circadian disruption, other measures of endocrine disruption (i.e., CAR slope or mean diurnal cortisol), or measures of immune disruption.

In analyses exploring associations between measures of psychosocial stress and physiological disruption, patients with lower income had less rhythmic circadian patterns of activity (i.e., greater circadian disruption). Patients with lower income had less healthy cortisol slope profiles (i.e., flatter slopes). Therefore, it is likely that individual 
socioeconomic status may serve as a source of stress in our sample, and in turn, may influence circadian and endocrine physiology.

Additionally, greater secretion of inflammatory/ $\mathrm{T}_{\mathrm{H}} 1$-type cytokines (i.e., IL-6, IL12 , IFN- $\gamma$ ) was observed in younger patients for analyses testing relationships between psychosocial stress and physiological disruption. It is possible that age may affect immune function in women with breast cancer. Research would benefit from exploration of potential differences in immune function between younger and older breast cancer patients, which may aid in the understanding of the difference in the epidemiology and prognosis of breast cancer in younger women.

Our analyses of psychosocial and physiological variables as prognostic indicators of progression-free survival produced no significant findings.

A fair amount of cancer research has focused on the relationships between psychosocial stress, physiological disruption, and disease progression; however, life context (e.g., ethnicity and neighborhood-related stressors) has rarely been explored within this framework. These novel data provide an opportunity to explore multiple types of psychosocial stress (i.e., ethnicity, neighborhood stress, and cancer-related stress) and their relationship with physiological disruption measures (i.e., circadian, endocrine, and immune disruption). By exploring these varying facets of stress, one can begin to understand which domains may be most salient in presurgical breast cancer patients and can provide suggestions for medical and psychological clinical interventions. 


\section{Associations Between Measures of Psychosocial Stress: Ethnicity was correlated with neighborhood stress, but not cancer-related stress. Neighborhood stress was not correlated with cancer-related stress (Figure 2).}

We conceptualized neighborhood stress as a composite of four variables related to socioeconomic status (i.e., percentage of households below the poverty line, median household income, percentage of single-parent homes, and percentage of renter-occupied housing in the neighborhood). Thus, we expected associations of our neighborhood stress measure with indicators of poverty. As expected, this study revealed that, in this sample of presurgical breast cancer patients, ethnicity was related to neighborhood stress. These findings are consistent with previous literature that indicates that, in the United States, ethnic minorities live in areas with concentrated poverty (Jargowsky \& Yang, 2006; Lichter, Parisi, \& Taquino, 2012). Additionally, African Americans have higher rates of individuals of living in areas of concentrated poverty compared to $\mathrm{NH}$ Whites (Lichter et al., 2012).

Neither ethnicity nor neighborhood stress was associated with cancer-related stress. These findings are inconsistent with previous literature regarding cancer-related stress symptoms across ethnicities. In a study of women who had been diagnosed with breast cancer in the prior two to three months, African American women were more likely than White women to endorse traumatic symptoms related to the cancer diagnosis and met criteria for PTSD (Vin-Raviv et al., 2013). A large proportion of participants in the study had completed surgery (i.e., lumpectomy or mastectomy) at baseline (VinRaviv et al., 2013). Therefore, it is possible that the discrepancy in the timing of the administration of the cancer-related stress assessment in our study compared to the study 
by Vin-Raviv and colleagues (2013) may explain the differences in findings. This may be due to African Americans experiencing differences in treatment and expectations for recovery. African Americans are often diagnosed with more adverse cancer pathology (Middleton et al., 2003), which is likely to need more intensive intervention and could make recovery more difficult. African American breast cancer patients have reported having a lack of information regarding treatment protocols and expectations regarding side effects (Lackey, Fates, \& Brown, 2001), which could increase stress following surgery. Furthermore, African Americans often rely on support from extended family and friends to cope; however, a sample of African American women with breast cancer reported that it was difficult to receive the support they needed due to their families own difficulties with coping (Ashing-Giwa et al., 2004). Furthermore, patients with colorectal cancer reported feeling as though their cancer was a burden on family, which was related to lower perception of physical health improvement (Kim, Shaffer, Rocha-Lima, Milton, \& Carver, 2016). Therefore, a lack of adequate support, or feeling as though one is a burden on family may contribute to increases in stress following the receipt of cancer treatment.

We expected to see associations of cancer-related stress with ethnicity and neighborhood stress. This hypothesis was based on the assumption that African Americans and those living in neighborhoods with lower socioeconomic resources would be more disadvantaged, possibly with less access to healthcare, which would translate to greater stress regarding their diagnosis. Lazarus and Folkman's (1984) theoretical model of stress and coping may help in understanding discrepancies between our presurgical breast cancer sample from other post-surgical samples. Women face new cancer-related 
challenges throughout the cancer trajectory (e.g., surgery, treatment, recovery, negotiating the medical system, fear of recurrence, and mortality). It is possible that they may experience an imbalance between stress and their resources to cope with demands, and that this imbalance may be greater after beginning treatment. Therefore, our lack of associations may be due to the assessment period being prior to initiation of any treatment interventions (e.g., surgery). It may be that over time, as the participants in the study adjust to the treatment and associated physical, social, and emotional changes, cancer-related stress may change. It is possible that ethnicity and neighborhood stress may play a role in those changes, particularly when taking into consideration disadvantages seen in ethnic minorities and those living in neighborhoods with high stress (e.g., fewer resources). However, in this study, women's ethnicity and current neighborhood status appeared unrelated to their perception of stress related to cancer.

\section{Associations Between Psychosocial Stress and Physiological Disruption}

\section{African American ethnicity was not related to circadian disruption,} endocrine disruption, or immune disruption (Figure 3). Ethnicity was not significantly related to measures of physiological disruption in this study. No research has explored ethnic differences in rest/activity rhythms. Given that we have exhausted the options in the literature that applies to this study, we compare our sample to those exploring ethnic differences in sleep, which is likely correlated with rest/activity rhythms. Sleep research has shown ethnic differences in sleep architecture (Rao et al., 1999), total sleep time (Hale \& Do, 2007; Lemola et al., 2013; Profant et al., 2002), sleep efficiency (Lemola et al., 2013); daytime sleepiness (Ancoli-Israel et al., 1995; Chen et al., 2015); and difficulties falling (Ancoli-Israel et al., 1995; Jean-Louis et al., 2000) and staying 
asleep (Jean-Louis et al., 2000). Given these studies explored sleep, which is fundamentally different than rest/activity rhythms, this may explain why we did not observe a relationship with ethnicity, while other studies assessing sleep did. Furthermore, it does not appear that there are published studies that have explored ethnicity and either sleep or rest/activity relationships in cancer samples. It is possible that within this sample of breast cancer patients, circadian rhythms may not be influenced by ethnicity. This could be due to additional factors seen in patients with cancer, particularly those with active tumors, which explain greater variance in circadian disruption than ethnicity.

The lack of a significant relationship between ethnicity and endocrine disruption is inconsistent with previous literature that has shown salivary cortisol secretion and diurnal cortisol slope dysregulation in African Americans, but not NH Whites. Specifically, among healthy young to middle-aged women, African Americans had flatter diurnal cortisol slopes (Cohen et al., 2006); this relationship was also observed in middleaged to older adult women (McCallum et al., 2006). Hispanic middle to older-aged women had significantly flatter slopes compared to NH Whites in a sample consisting of caregivers and non-caregivers (Gallagher-Thompson et al., 2006). In a sample of pregnant women, African Americans had flatter cortisol slopes than their Hispanic counterparts (Suglia et al., 2010). Among healthy adults, African Americans had flatter slopes and lower waking cortisol levels (Hajat et al., 2010). Similar findings have been observed in African American and Hispanic adolescents (DeSantis et al., 2007). However, research did not find ethnic differences in endocrine disruption in a populationbased study of middle-aged men and women utilizing urinary cortisol (Masi et al., 2004). 
Inconsistences with prior research may be related to differences in measurement or analysis of data (e.g., cortisol slope calculation).

Within our sample, the lack of an association between ethnicity and endocrine disruption could be due to other factors that may account for endocrine disruption. For example, previous research showing ethnic differences in overall cortisol secretion indicated that perceived stress may explain ethnic differences (Bennett et al., 2004). Additionally, in a community-based sample of adult men and women, African American and Hispanic/Latino participants had flatter slopes associated with increased psychosocial stress (DeSantis, Adam, Hawkley, Kudielka, \& Cacioppo, 2015). It is possible that the perception of stress related to ethnicity (e.g., racial/ethnic discrimination) may be related to endocrine disruption, and only measuring ethnicity may not fully capture this effect. Limited research has explored ethnic differences in immune disruption and has shown that, in healthy adults, lower levels of IL-8 and G-CSF (Mayr et al., 2007) and higher levels of IL-6 and CRP have been observed in African Americans compared to NH Whites (Stowe et al., 2010). We did not observe ethnic differences in immune variables. The inconsistences with prior research may be due to a number of different factors, such as methodological differences, differences in blood collection (i.e., serum vs. plasma), and differences in collection time (Mayr et al., 2007; Stowe et al., 2010). Additionally, one study's sample was only men (Mayr et al., 2007), which may also explain the differences between their findings and this study. Indeed, there are sex differences in immune function (Nakamura et al., 2000; Oertelt-Prigione, 2012). Other factors may better explain differences in immune functioning than ethnicity. For 
example, it is possible that the ethnic differences observed in plasma CRP may be driven by body mass index and obesity (Stowe et al., 2010).

In this study, ethnicity is conceptualized as a potential psychosocial stressor. The lack of associations observed between ethnicity and physiological disruption may indicate that factors related to ethnicity (e.g., racial/ethnic discrimination) may better capture ethnicity as a stressor. Ethnicity-related factors, such as racial/ethnic discrimination can likely be conceptualized as a chronic stressor. Among adolescents, increased perceived discrimination was associated with elevated cortisol levels throughout the day (Huynh, Guan, Almeida, McCreath, \& Fuligni, 2016) and ethnicity moderated the relationship between perceptions of discrimination and diurnal cortisol slope (Zeiders, Hoyt, \& Adam). Additionally, African American young women reporting one or two discriminatory experiences had higher levels of CRP than those who had not had these experiences (Cunningham, et al., 2012). Therefore, factors closely related to ethnicity, such as perceived discrimination, may contribute to changes in physiological disruption.

Neighborhood stress was associated with elevated diurnal mean cortisol, but not with circadian or immune disruption (Figure 4). Neighborhood stress was significantly associated with diurnal cortisol mean, a measure of endocrine disruption. Previous research has shown that individuals living in neighborhoods with increased levels of perceived stressors had an increase of cortisol throughout the day and evening, starting three hours after waking, and lower overall cortisol mean (Karb et al., 2012). However, these stressors were not socioeconomic in nature, as Karb and colleagues (2012) explored perceived neighborhood violence, safety, physical hazards, and quality 
of neighborhood service and observed homicides, robberies and burglaries, physical disorder and deterioration, vacant lots and houses, and stress conditions (Karb et al., 2012). Neighborhood violence has been shown to be associated with low waking cortisol, and a "weaker, less consistent" association with neighborhood poverty and cortisol slope (Do et al., 2011, p. 630). Furthermore, research has shown associations between total serum cortisol and a neighborhood stress index comprised of unemployment rates, poverty, female head of households with dependent children, and vacant housing (DulinKeita et al., 2012).

The differences in findings in this study and the literature may be due to differing methods of measurement of cortisol, as well as differences in what neighborhood stressors were explored. As demonstrated among adolescents living in neighborhoods perceived as discriminatory (Huynh et al., 2016), it is possible that living in a neighborhood that can be considered dangerous or socioeconomically stressful has an impact on physiological disruption by increasing secretion of cortisol. Within this sample of women, those living in areas with high neighborhood stress may have been experiencing these stressors for an extended period, which could manifest in stress that is chronic in nature. It may be that chronic neighborhood stress functions similarly to other chronic stressors, contributing to increasing overall cortisol production in response to the stress, which, in turn, may have harmful effects on health.

Neighborhood stress was not significantly related to circadian disruption. No data on neighborhood stress and rest/activity rhythms is available. For comparison purposes, neighborhood stress has been shown to be related to sleep (Hale et al., 2010; Hill et al., 2009; Steptoe et al., 2008). It is important to note that while there is a probable 
correlation between rest/activity rhythms and sleep, these are different parameters. Rest/activity rhythms are measured over multiple days and are based on circadian patterns of activity. Research has focused exclusively on relations with sleep and neighborhood disorder and crime. The differences in measures of neighborhood stress, as well as assessing sleep, rather than rest/activity rhythms, may explain the discrepancies in findings seen in previous research and this study. One study exploring the relationship between sleep and neighborhood stress found that this association was no longer significant after adjusting for self-reported health (Steptoe et al., 2008).

Neighborhood stress was not significantly related to immune disruption. While no significant findings were seen in this study, this is an exciting area for further research as the literature on stress and immune function is vast, and one article exploring the relationship between neighborhood stress and health suggested that immune function may mediate this relationship (Steptoe \& Feldman, 2001). In a study of NH Whites and African Americans, middle to older age adults living in more deprived neighborhoods had greater odds of inflammation than those who live in less deprived areas (Dulin Keita, Judd, Howard, Carson, Ard, \& Fernandez, 2014). Additionally, greater neighborhood deprivation was related to greater levels of IL-6, CRP, and fibrinogen (i.e., a protein for clotting; Nazmi, Roux, Ranjit, Seeman, \& Jenny, 2010). Exploring the possible role of neighborhood stress on immune function within a cancer population could yield significant findings that may help us understand how neighborhood stress may affect physiology relevant to cancer. Additionally, in this study we explored relationships in the context of an active tumor (i.e., presurgically). It is possible that tumor influences on immune disruption could introduce variance that could mask stress-immune effects. 
To understand relationships between neighborhood stress and physiological disruption, the length of time the individual lived in a particular neighborhood should be considered. This is relevant in determining whether neighborhood stress could be considered an acute or chronic source of stress for the individual. If neighborhood stress for the individual is acute in nature, we may not see effects on physiological disruption. If the individual recently moved from a neighborhood of high stress to an area of low stress, we may see significant findings given the variation in amount of time spent in the current neighborhood and the potential for sustained effects from living in the prior neighborhood. However, it is possible in this scenario to not see associations between neighborhood stress and physiological disruption due to possible improved access or resources if one moves from a neighborhood with high stress to a neighborhood with low stress. Access to resources (e.g., transportation, medical facilities) may explain relationships observed when exploring the effects of neighborhood stress.

A theoretical framework has been developed to understand cancer disparities in relation to neighborhood chronic toxic stress exposure (DeGuzman \& Schminkey, 2016). This model proposes that the social and built environment; which is a product of historical events (e.g., racial segregation) and neighborhood associated chronic toxic stressors (e.g., violence), influence health behaviors, mental health, and genomics (i.e., genomic epigenetic modifications and DNA damage); which in turn, affect cancer outcomes (DeGuzman \& Schminkey, 2016). Considering this model of the complex effects of neighborhood stress on cancer increases the interest regarding the influence of neighborhood stress of physiological disruption, as well as the potential associated effects on outcomes (e.g., quality and quantity of life) in cancer patients. 


\section{Cancer-related stress was associated with greater diurnal cortisol}

\section{rhythmicity. This association may be explained by adaptive avoidance of cancer-}

related thought. Interestingly, within our sample of presurgical breast cancer patients, cancer-related stress was related to steeper, more rhythmic cortisol slopes. Post hoc analyses revealed a significant relationship between avoidance, but not intrusions, and these "healthier" diurnal cortisol slopes. The avoidance subscale includes staying away from reminders; avoidance of emotions; thought suppression; not trying to "deal with" the negative emotions and difficulties; trying to remove an event from memory; and not talking about the event. It is possible that within this sample of women, these strategies of avoidance provided a buffering effect for the acute stressor that is a breast cancer diagnosis. Research from our lab exploring daily intrusions and daily avoidant coping relationships with diurnal cortisol slope did not emerge as significant in this same sample of women (Dedert et al., 2012). Differing strategies in data collection included daily intrusions and daily avoidant coping measured each day over three days, during the cortisol collection in the study by Dedert and colleagues (2012). Additionally, avoidant coping was measured using subscales of the Brief COPE measure (Dedert et al., 2012), whereas this study utilized the IES. The difference in data collection may account for the differences in findings.

The presurgical phase includes medical evaluations and preparation for surgery and is often associated with significant mood disturbance and elevated distress in relation to sleep difficulties, fatigue, and concentration difficulties in women with breast cancer (Cimprich, 1999). Further, the pre-treatment period was the most stressful for women with breast cancer (Culver, Arena, Antoni, \& Carver, 2002; Northouse, 1998) 
with the distress decreasing over time following initial diagnosis (Compas \& Luecken, 2002; Culver et al., 2002; Burgess et al., 2005). Among men with prostate cancer who had completed treatment, avoidant coping was found to be related to flatter diurnal cortisol slopes at baseline and four-month follow-up (Hoyt et al., 2014). Given the differences between our findings and those who have completed treatments, it may be that avoidance provides a safeguard to changes in circadian regulation during the acutely stressful presurgical period, but may have detrimental effects on physiology during the post-treatment period or in response to chronic stress.

Cancer-related stress was not significantly related to circadian disruption. Limited research has explored the relationship between cancer-specific distress, a measure of intrusions and avoidance, and circadian rhythms. Research conducted on this same sample of women by our lab showed associations between daily intrusions and daily avoidant coping with consistency of rest/activity rhythms, daytime sedentariness, and nighttime restlessness (Dedert et al., 2012). However, the collection strategy for cancerrelated stress (i.e., intrusions and avoidance) differed, in that this study explored cancerrelated stress at one-time point, baseline, whereas the study by Dedert and colleagues (2012) explored these variables daily over the same three days that actigraphy was collected. The average (SD) days between our collection at baseline and the actigraphy collection were 0.167 days (3.975), with a median of one day. The discrepancy in findings may be explained by day-to-day variability. Therefore, it may be that nuanced variations in daily levels of intrusions and avoidant coping are better predictors of circadian disruption than obtaining data on a patient's average levels over the past week. It may be that in the days following a cancer diagnosis, cancer-related stress may increase 
as the individual has had more opportunity to reflect on their diagnosis and the implications that the diagnosis and treatment will have on their quality and quantity of life. Additionally, it is possible that individually, either intrusions or avoidance may be better associated with circadian disruption than the combination of the two.

Cancer-related stress was not associated with other measures of endocrine disruption (i.e., CAR slope and diurnal cortisol mean). Research completed by our lab, using the same sample of women, did not observe associations between daily intrusions or daily avoidant coping with CAR slope or diurnal mean cortisol (Dedert et al., 2012). Other previous research has shown that intrusions and avoidance might be related to cortisol alterations. One meta-analysis indicated that following a traumatic event, there might be increases in cortisol production, but generally, cortisol levels are lower in individuals with PTSD (Yehuda, 2002). This contrasts with our sample as PTSD develops after at least one month of symptoms following a traumatic event; whereas, data was collected in our sample an average (SD) of 19.21 (20.82) days after diagnosis, with a median of 14 days. Within a sample of healthy women with no personal or family histories of breast cancer, breast cancer specific intrusions were related to increased cortisol responses to acute stressors at work (Dettenborn et al., 2006).

Cancer-related stress was not significantly related to immune disruption. Prior research has shown increased cancer-related stress to be related to inhibition of cellular immune response (Andersen et al., 1998), decline in natural killer cell activity and stimulated IFN- $\gamma$ (Von Ah et al., 2007), and elevations of plasma IL-1R $\alpha$ (Mundy-Bosse et al., 2011) among post-surgical breast cancer patients. In metastatic breast cancer patients, depression was related to suppressed immunity (Sephton et al., 2009). 
Additionally, in ovarian cancer patients, increased vegetative depression was associated with elevated plasma IL-6 (Lutgendorf et al., 2008) and increased helplessness was related to increased serum VEGF (Lutgendorf et al., 2002). It is possible that the lack of findings in this study may be due to differences in measurements of distress (e.g., cancer-related stress vs. depression) and immune function (e.g., serum vs. stimulated cytokines), as well as timing of data collection (e.g., presurgical vs. post-surgical).

In this study, the timing of the measurement of variables, including cancer-related stress and physiological disruption, was prior to any surgical procedure. Receiving a cancer diagnosis can be regarded as an acutely stressful period, and it may be that this period of stress has not been experienced for a long enough amount of time to be related to or cause changes in physiological disruption, which could explain the lack of findings. It is also possible that in the short term, avoidance, one aspect of cancer-related stress, may provide effects that protect against changes to physiology. Avoidance has been conceptualized to include denial (Taylor \& Stanton, 2007), which is "trying to push the reality of the situation away" (Carver, 1997, p.98). In addition to denial, avoidance also includes repression, distraction, and disengagement (Carver, Scheier, \& Weintraub, 1989). In a study of women with breast cancer, within one week following mastectomy, denial was associated with lower levels of stress, which indicates that denial may be a short term adaptive coping response to a diagnosis of cancer (Watson, Greer, Blake, \& Shrapnell, 1984). Denial was also seen in a sample of lung cancer patients, which the authors suggest is normal and highlights a need to protect one's self from the stress associated with a cancer diagnosis, and that over time use of denial and distraction decrease (Vos, Putter, van Houwelingen, \& de Haes, 2008). It is possible that maintaining 
as much normalcy as possible during the early phases of the cancer trajectory may prove beneficial in managing the short-term stress of a diagnosis. However, longitudinal research within a cancer population may elucidate the risks and benefits of various types of coping throughout cancer diagnosis, treatment, and survivorship. Indeed, in a sample of women with early stage breast cancer, denial and a fighting spirit were significantly more likely to be predictive of five-year survival than stoic acceptance of hopelessness/helplessness (Greer, Morris, \& Pettingale, 1979).

\section{No predictors of progression-free survival emerged.}

Measures of psychosocial stress (i.e., ethnicity, neighborhood stress, and cancerrelated stress) were not significantly related to medical outcomes of cancer (progressionfree survival) in this study. These findings are inconsistent with previous literature that shows significant ethnic disparity in cancer survival; however, the previous research has largely been epidemiological in nature.

Disparities in survival rates among NH White and African American breast cancer patients may be attributed to racial differences in patterns of care (Diehr et al., 1989), treatment options and choices (Bradley, Given, \& Roberts, 2002; Hershman et al., 2005), barriers to access to care (DeSantis, Siegel, Bandi, \& Jemal, 2011), noncompliance with therapy, and incomplete treatment adherence, which may be related to socioeconomic status (Baquet, Mishra, Commiskey, Ellison, \& DeShields, 2008). However, it has been suggested that the ethnic disparity in mortality may be due to factors other than those related to access to care (Chlebowski et al., 2005). For example, ethnic differences in genetics, which may also be attributed to neighborhood stress, could explain differences in cancer outcomes (DeGuzman \& Schminkey, 2016). Therefore, 
cross-sectional, experimental, and longitudinal research would be beneficial to help elucidate these disparities.

There are possible explanations for the lack of significant findings in this study. It is possible that the sample size for those who have had recurrent disease or have passed was too small to detect significant effects. With survival analyses, it is suggested that at least $50 \%$ of participants should have experienced a recorded event (i.e., recurrence or death in this study). This study only had $30 \%$ of participants that had recurrent disease or passed; therefore, this may have been too small a proportion to detect significant effects. Another, related, possibility is that of missing data. Considering the low number of participants for whom survival data were available $(n=16)$, analyses may have been underpowered.

Measures of physiological disruption (i.e., circadian, endocrine, and immune) were not significantly related to progression-free survival. These findings are inconsistent with previous literature that have shown rest/activity rhythms (Innominato et al., 2009; Lévi et al., 2014; Mormont et al., 2000), diurnal cortisol slope (Cohen et al., 2012; Schrepf et al., 2015; Sephton et al., 2013; Sephton et al., 2000), VEGF (Jin-no et al., 1998; Karayiannakis et al., 2002), and IL-1 $\beta$ (Barber et al., 2000) to be associated with shorter survival in various cancer populations. As described above, it is possible that the small sample size provided too little statistical power to reveal significant findings. In addition, it is possible that no significant findings were observed because this sample of women was presurgical. It is plausible that presurgical cancer patients have not had significant changes to physiological disruption markers that affect survival (Cash et al., 2015). If this is the case, these results could be promising in that interventions targeted 
during the presurgical period, or prior to neoadjuvant treatment may delay physiological disruption, or weaken the effects of physiological disruption on survival.

Extensive research has shown that various psychosocial interventions decrease stress related to the experiences of cancer diagnosis and treatment, which may improve physiology throughout and following treatment (Antoni, 2013). However, prehabilitation research is a nascent area within the cancer rehabilitation continuum, which focuses on the time between diagnosis and the beginning of treatment (Silver, 2015). Preoperative interventions can focus on physical functioning, nutrition, psychological health, and stress management, which may influence physiological and psychological outcomes in patients with cancer (Silver, 2015). Indeed, a review of the cancer prehabilitation literature revealed that preoperative psychological interventions had significant effects on immune function, psychological outcomes, somatic symptoms, and quality of life (Tsimopoulou et al., 2015).

Associations have been observed between psychosocial stress and physiological disruption with another cancer outcome, quality of life (QOL). In a study exploring the relationships between distress and QOL with this same sample of women, an association between cancer-related stress and QOL, as well as ethnic differences were observed (Rebholz et al., 2016). For example, NH White women reported poorer emotional QOL in relation to cancer-related distress, which may indicate that African American women utilize more effective means of coping or rely on or experience more positive social support (Rebholz et al., 2016). Research has shown that African Americans are more likely to use spirituality in coping with cancer-related stressors (Culver, Arena, Antoni, \& Carver, 2002). Additionally, research has shown relationships between circadian 
disruption (Innominato et al., 2009) and endocrine disruption (Carlson, Speca, Patel, \& Goodey, 2004; Miller, Ancoli-Israel, Bower, Capuron, \& Irwin, 2008) and QOL. IL-6 has also been shown to be associated with fatigue, which strongly affects QOL in cancer patients (Cleeland et al., 2003; Seruga, Zhang, Bernstein, \& Tannock, 2008).

\section{Low-income patients had poor circadian coordination.}

In analyses exploring associations between psychosocial stress and circadian disruption, patients reporting lower income had greater circadian disruption, indicated by less rhythmic rest/activity patterns and greater daytime sedentary behavior. Within this same sample of women, lower income was associated with greater rest/activity disruption in tests of associations with daily intrusions and daily avoidant coping (Dedert et al., 2012). Income likely serves as a significant source of stress, which may negatively impact rest/activity rhythms. Therefore, income disadvantage appears to play a role in circadian disruption and should be further explored to understand the mechanisms driving this association.

Additionally, income emerged as a significant predictor in the analysis assessing the relationship between neighborhood stress and endocrine disruption, such that patients reporting lower income levels had flatter diurnal cortisol slopes, which are indicative of a less healthy profile. In a large study of older adults, financial insecurity explained a portion of the variance in the relationship between social position and cortisol slope (Kumari et al., 2010). Lower socioeconomic status was related to lower waking cortisol levels and flatter cortisol slopes in the early portion of the day (Hajat et al., 2010). In contrast, higher income has been shown to be related to steeper cortisol decline over the day (Wang et al., 2014). The findings of this study support previous literature that 
income, one measure of an individual's socioeconomic status, may serve as a source of stress that influences endocrine physiology. Disadvantage related to low income is a significant stressor to many individuals and is potentially more salient to those affected by cancer as they face treatment decisions that are likely to influence various areas of life related to income, such as the ability to work or care for family and the home. Continued research is needed to better understand the role income plays in chronic endocrine changes, as socioeconomic disadvantage may influence cancer progression.

\section{Younger patients had greater immune disruption.}

In the analyses assessing the associations of psychosocial stress and immune disruption, younger patients had greater secretion of inflammatory/ $\mathrm{T}_{\mathrm{H}} 1$-type cytokines (i.e., IL-6, IL-12, IFN- $\gamma$ ). Research has shown that younger women with breast cancer are often diagnosed at a later stage and have adverse pathology as evidenced by higher grade tumors, negative hormone receptor status, and overexpression of HER2 (Assi et al., 2013). Research has shown IL-6 levels increase as one ages in samples of older adults (Kiecolt-Glaser, Preacher, MacCallum, Atkinson, Malarkey, \& Glaser, 2003), healthy subjects (Wei, Xu, Davies, \& Hemmings, 1992), and women (Haden, Glowacki, Hurwitz, Rosen, \& LeBoff, 2000). While these findings are in the opposite direction as those we observed, our results are not surprising given the more difficult prognosis of younger breast cancer patients and the likely associated stress. Further research should explore relationships between age and these cytokines in breast cancer patients to understand how age may influence inflammation/ $\mathrm{T}_{\mathrm{H}} 1$-type immunity. Better understanding this potential relationship may lead to avenues for treatment that could improve outcomes for younger breast cancer patients. 


\section{Strengths and Limitations}

This study represents the first examination of multiple types of psychosocial stress and physiological disruption in presurgical breast cancer patients. By examining ethnicity, the impact of the environment (i.e., neighborhood), one's perception of stress related to a cancer diagnosis, and changes in physiology through the use of subjective self-report measures, census data, and objective measures allowing for a more comprehensive evaluation of stress. Therefore, by collecting data on multiple sources of stress measured with different methods, it is possible to explore the importance of both psychosocial stress and physiological disruption during the cancer process.

Utilizing census data to study the effects of neighborhood stress is an approach that allows for a more accurate reflection of the status of the neighborhood, reflected as a census tract, compared to obtaining self-report of one's perceptions of their neighborhood stress. Both methods of measurement hold merit to use in research; however, by utilizing census data, it is possible to obtain an objective understanding of neighborhood stress. By conducting a principal components analysis (PCA), it was possible to explore four variables that loaded onto one factor, neighborhood stress. This helped to limit the number of analyses run when exploring the effects of neighborhood stress on physiological disruption and medical outcomes of cancer.

Measuring circadian disruption with two indicators (i.e., rest/activity rhythms and diurnal cortisol rhythms) allows for these two types of circadian rhythms to be measured via separate and objective methodology (i.e., activity levels and salivary cortisol levels, respectively). Actigraphy data may be more likely to be influenced by exogenous factors, which, for example, may include cues from the social environment (e.g., light exposure, 
meal timing). Diurnal cortisol measurement depicts endogenous rhythms regulated by the SCN.

For multiple reasons, a novel aspect of this study is the timing of recruitment and data collection, which occurred relatively soon after the cancer diagnosis and prior to surgical treatment. There is a dearth of research during this time point, with the majority of studies focusing on patients during adjuvant treatment or those with survivor status (i.e., greater than or equal to five years disease-free). By assessing mental and emotional state and physiology prior to surgery and other treatments, it is possible to gain an understanding of these variables with an active tumor present in the body. Our data were not clouded by variability resulting from physically and emotionally demanding cancer treatments. Additionally, by conducting assessments at this time point it is possible to assess psychosocial and biological constructs before the long-term cancer treatment process alters them. By assessing physiology presurgically and prior to other types of treatment (e.g., neoadjuvant chemotherapy and radiation therapy), systems have not yet been dysregulated by these medical interventions.

It is noteworthy that this sample was ethnically diverse. The ethnic breakdown of the sample was approximately 58\% NH White and about 37\% African American. There were an additional three participants, two reporting their ethnicity as Native American and one reporting her ethnicity as Asian American. These three individuals were excluded from the analyses utilizing ethnicity as a predictor, due to potential cultural differences in psychosocial stress and physiological disruption. Rather than grouping the individuals into a general minority category, it is preferable to consider the different cultural factors of each specific ethnic group. Additionally, given the small sample size of 
Native Americans and Asian Americans, it was necessary to exclude them from the analyses.

The overall relatively small sample size of the study is itself another limitation. A larger study would provide greater power and allow for the detection of small magnitude effects (i.e., phenomena with smaller effect sizes). Additionally, there were missing data, which coupled with the small sample size created problems with the survival analyses. No survival analyses emerged as significant. This is likely, in part, due to the low number of participants with a recorded event (i.e., 30\% of participants with recurrence or death) once predictor-missing data were accounted for. The number of events was already low for survival analyses and once the removal of those with missing data, the number was even lower.

This study was exploratory in nature. This was an approach that was taken to provide direction for the generation of hypotheses in future research studies, rather than to confirm hypotheses. Therefore, the results presented here should be considered with caution as they are not generalizable outside this sample. Given the large number of analyses that were run, results must be interpreted with great caution. Our many nonsignificant findings could be attributable to the small sample size, or may be due to differences in data collection and statistical tests compared to previous studies. However, given the exploratory nature of the study, the results should serve as a guide for the development of future research and relationships that were not significant should be explored further.

\section{Future Directions}


The results of this study point to a number of directions for additional research to explore. It is reasonable to think that some aspects of psychosocial stress may influence physiological disruption early in the cancer trajectory, even before any surgical or medical intervention. Further studying the impact of psychosocial stress on physiological disruption within cancer populations may provide an even greater understanding of how stress affects physiology when an individual experiences chronic disease.

It may be beneficial to consider the manner in which the psychosocial factors are measured in research. This study used a dichotomous self-report of ethnicity; however, an improved manner for measuring ethnicity would be to utilize a validated measure, such as the Multigroup Ethnic Identity Measure (MEIM; Phinney, 1992; Phinney \& Ong, 2007). The MEIM would be more beneficial than a dichotomous or categorical self-report of ethnicity, as it would allow for a better understanding of one's ethnic identity, sense of belonging with their ethnic origin, and the possible impact that this has on physiological disruption.

With regard to neighborhood stress, it would be useful for future research to include an index, as this study did, to assess various factors that may impact physiological disruption. By utilizing an index of neighborhood stress, researchers would be able to explore various areas, such as neighborhood poverty levels, median neighborhood income, and other factors, that may contribute to the difficulty of living in a particular neighborhood. This could allow for a better understanding of what individual factors may contribute to changes in physiological disruption. Additionally, knowledge would be gained as to the impact of the cumulative nature of environmental stressors within the neighborhood context. 
Mediator and moderator variables were not explored in this study for multiple reasons, including the small sample size and that the study sought to utilize exploratory analyses to gain a better understanding of direct relationships between psychosocial stress and physiological disruption. However, the role of mediators and moderators are essential in better understanding the relationships between psychosocial stress and physiological disruption. Potential mediators and moderators that would be advantageous to study include access to and utilization of healthcare, social support, coping resources, which may include active coping strategies, religiosity or spirituality, and meaning making or benefit finding. While this list is not exhaustive, it highlights potential variables of significance.

While this study employed a cross-sectional design, longitudinal research could illuminate how psychosocial stress and physiological disruption change over time. It is likely that there is a time during the cancer trajectory that psychosocial stress and physiological disruption are stronger predictors of one another, as well as stronger predictors of survival. A longitudinal design can allow for hierarchical linear modeling, which can illuminate the period during which psychosocial factors most strongly predict cancer outcomes. From gaining this type of knowledge, substantial implications for medical and psychological intervention would result. By knowing what psychosocial stress or physiological disruption factor is a stronger predictor at a particular time during one's cancer journey, more assessments could be completed during that period, which might lead to either psychological or medical interventions to improve the quality or extend the quantity of life for individuals with cancer. 
Future research that investigates psychosocial stress would benefit from including ethnicity and neighborhood stress, in addition to an individual's perceived stress related to their diagnosis. With these additions, research will more adequately explore various stressors that may play a role in physiological disruption. By continuing to explore these associations, research can begin to understand more about the driving factors of physiological disruption. This may help to identify the most predictive aspects of stress, identify future medical and clinical treatments, and delay or prevent adverse changes to physiology/health outcomes.

Research should continue to explore the mechanisms of stress effects on cancer survival. Research in which assessments are completed prior to surgery or other medical intervention is crucial. Research of this nature may shed light on the effects of an active tumor on physiological disruption and the possible implications throughout the cancer trajectory, as well as the individual's lifespan. If associations are seen between early onset psychosocial stress, physiological disruption, and survival; we may discover new markers to target early in the cancer process.

The current study explored novel relationships between psychosocial stress and physiological disruption within a sample of women that were awaiting surgery for breast cancer. The association between neighborhood stress and endocrine physiology emphasizes the importance of improved resources and access within disadvantaged neighborhoods, which would likely reduce stress and improve resident's health. An unexpected relationship between avoidance and healthier diurnal cortisol slopes indicates that avoidance may serve to help maintain normalcy during the acutely stressful, chaotic, presurgical period. These intriguing findings highlight the importance 
of psychosocial intervention while awaiting treatment for cancer. Prehabilitation interventions, when psychosocial stress is probably most salient to a woman with cancer, are worthy of investigation. Research of this nature could aid in identifying methods for improvement of quality, and possibly quantity, of life for women with breast cancer. 


\section{REFERENCES}

Aardex. (2001). MEMS IV. Union City, CA.

Abercrombie, H. C., Giese-Davis, J., Sephton, S., Epel, E. S., Turner-Cobb, J. M., \& Spiegel, D. (2004). Flattened cortisol rhythms in metastatic breast cancer patients. Psychoneuroendocrinology, 29, 1082-1092.

Addinsoft. (2014). XLSTAT. New York, NY.

Adem, C., Reynolds, C., Ingle, J. N., \& Nascimento, A. G. (2004). Primary breast sarcoma: clinicopathologic series from the Mayo Clinic and review of the literature. British Journal of Cancer, 91, 237-241. http://doi.org/10.1038/sj.bjc.6601920

Adler N.E., Boyce W., Chesney M.A., Folkman S., \& Syme S. (1993). Socioeconomic inequalities in health: No easy solution. JAMA, 269, 3140-3145. http://doi.org/10.1001/jama.1993.03500240084031

Alexander, K., Entwisle, D., \& Olson, L. (2014). The Long Shadow: Family Background, Disadvantaged Urban Youth, and the Transition to Adulthood. Russell Sage Foundation.

American Psychiatric Association. (2013). Diagnostic and Statistical Manual of Mental Disorders (DSM-5®). American Psychiatric Publication. 
Ancoli-Israel, S., Cole, R., Alessi, C., Chambers, M., Moorcroft, W., \& Pollak, C. P. (2003). The Role of Actigraphy in the Study of Sleep and Circadian Rhythms. SLEEP, 26, 342-392.

Ancoli-Israel, S., Klauber, M. R., Stepnowsky, C., Estline, E., Chinn, A., \& Fell, R. (1995). Sleep-disordered breathing in African-American elderly. American Journal of Respiratory and Critical Care Medicine, 152, 1946-1949. http://doi.org/10.1164/ajrccm.152.6.8520760

Ancoli-Israel, S., Liu, L., Marler, M. R., Parker, B. A., Jones, V., Sadler, G. R., ... Fiorentino, L. (2006). Fatigue, sleep, and circadian rhythms prior to chemotherapy for breast cancer. Support Care Cancer, 14, 201-209.

Andersen, B. L., Farrar, W. B., Golden-Kreutz, D., Kutz, L. A., MacCallum, R., Courtney, M. E., \& Glaser, R. (1998). Stress and immune responses after surgical treatment for regional breast cancer. Journal of the National Cancer Institute, 90, 30-36.

Andersen, B. L., Kiecolt-Glaser, J. K., \& Glaser, R. (1994). A biobehavioral model of cancer stress and disease course. American Psychologist, 49, 389-404. http://doi.org/10.1037/0003-066X.49.5.389

Andersson, I. (2005). Invasive Breast Cancer. In N. C. Gourtsoyiannis M.D. \& P. R. Ros M.D., MPH (Eds.), Radiologic-Pathologic Correlations from Head to Toe (pp. 757-766). Springer Berlin Heidelberg.

Antoni, M. H. (2013). Psychosocial intervention effects on adaptation, disease course and biobehavioral processes in cancer. Brain, Behavior, and Immunity, 30, Supplement, S88-S98. http://doi.org/10.1016/j.bbi.2012.05.009 
Antoni, M., Lutgendorf, S. K., Cole, S., Dhabhar, F., Sephton, S. E., McDonald, P., ... Sood, A. (2006). The influence of bio-behavioural factors on tumour biology: Pathways and mechanisms. Nature Reviews Cancer, 6, 240-248.

Armaiz-Pena, G. N., Cole, S. W., Lutgendorf, S. K., \& Sood, A. K. (2013).

Neuroendocrine influences on cancer progression. Brain, Behavior, and Immunity, 30, Supplement, S19-S25. http://doi.org/10.1016/j.bbi.2012.06.005

Arnaboldi, P., Lucchiari, C., Santoro, L., Sangalli, C., Luini, A., \& Pravettoni, G. (2014). PTSD symptoms as a consequence of breast cancer diagnosis: clinical implications. SpringerPlus, 3, 392. http://doi.org/10.1186/2193-1801-3-392

Ashing-Giwa, K. T., Padilla, G., Tejero, J., Kraemer, J., Wright, K., Coscarelli, A., ... \& Hills, D. (2004). Understanding the breast cancer experience of women: a qualitative study of African American, Asian American, Latina and Caucasian cancer survivors. Psycho-Oncology, 13, 408-428.

Assi, H. A., Khoury, K. E., Dbouk, H., Khalil, L. E., Mouhieddine, T. H., \& El Saghir, N. S. (2013). Epidemiology and prognosis of breast cancer in young women. Journal of Thoracic Disease, 5, S2-S8.

Balkwill, F. (2006). TNF- $\alpha$ in promotion and progression of cancer. Cancer and Metastasis Reviews, 25, 409-416. http://doi.org/10.1007/s10555-006-9005-3

Baquet, C. R., Mishra, S. I., Commiskey, P., Ellison, G. L., \& DeShields, M. (2008). Breast Cancer Epidemiology in Blacks and Whites: Disparities in Incidence, Mortality, Survival Rates and Histology. Journal of the National Medical Association, 100, 480-489. http://doi.org/10.1016/S0027-9684(15)31294-3 
Barber, M. D., Powell, J. J., Lynch, S. F., Fearon, K. C. H., \& Ross, J. A. (2000). A polymorphism of the interleukin-1 $\beta$ gene influences survival in pancreatic cancer. British Journal of Cancer, 83, 1443-1447. http://doi.org/10.1054/bjoc.2000.1479

Bauer, K. R., Brown, M., Cress, R. D., Parise, C. A., \& Caggiano, V. (2007). Descriptive analysis of estrogen receptor (ER)-negative, progesterone receptor (PR)-negative, and HER2-negative invasive breast cancer, the so-called triple-negative phenotype. Cancer, 109, 1721-1728. http://doi.org/10.1002/cncr.22618

Bennett, G., Merritt, M., \& Wolin, K. (2004). Ethnicity, education, and the cortisol response to awakening: a preliminary investigation. Ethnicity \& Health, 9, 337347.

Blackman, D. J., \& Masi, C. M. (2006). Racial and Ethnic Disparities in Breast Cancer Mortality: Are We Doing Enough to Address the Root Causes? Journal of Clinical Oncology, 24, 2170-2178. http://doi.org/10.1200/JCO.2005.05.4734

Blakeman, V., Williams, J. L., Meng, Q. J., \& Streuli, C. H. (2016). Circadian clocks and breast cancer. Breast Cancer Research, 18, 89. http://doi.org/10.1186/s13058016-0743-z

Boardman, J. D. (2004). Stress and physical health: the role of neighborhoods as mediating and moderating mechanisms. Social Science \& Medicine, 58(, 24732483. http://doi.org/10.1016/j.socscimed.2003.09.029

Bradley, C. J., Given, C. W., \& Roberts, C. (2001). Disparities in cancer diagnosis and survival. Cancer, 91, 178-188. http://doi.org/10.1002/10970142(20010101)91:1<178::AID-CNCR23>3.0.CO;2-S 
Bradley, C. J., Given, C. W., \& Roberts, C. (2002). Race, Socioeconomic Status, and Breast Cancer Treatment and Survival. Journal of the National Cancer Institute, 94, 490-496. http://doi.org/10.1093/jnci/94.7.490

Braun, L. (2002). Race, Ethnicity, and Health: Can Genetics Explain Disparities? Perspectives in Biology and Medicine, 45, 159-174. http://doi.org/10.1353/pbm.2002.0023

Broyles, S. T., Staiano, A. E., Drazba, K. T., Gupta, A. K., Sothern, M., \& Katzmarzyk, P. T. (2012). Elevated C-Reactive Protein in Children from Risky Neighborhoods: Evidence for a Stress Pathway Linking Neighborhoods and Inflammation in Children. PLOS ONE, 7, e45419. http://doi.org/10.1371/journal.pone.0045419

Burgess, C., Cornelius, V., Love, S., Graham, J., Richards, M., \& Ramirez, A. (2005). Depression and anxiety in women with early breast cancer: five year observational cohort study. British Medical Journal, 330, 702-705.

Butler, L. D., Koopman, C., Classen, C., \& Spiegel, D. (1999). Traumatic stress, life events, and emotional support in women with metastatic breast cancer: Cancerrelated traumatic stress symptoms associated with past and current stressors. Health Psychology, 18, 555-560. http://doi.org/10.1037/0278-6133.18.6.555

Calle, E. E., Rodriguez, C., Walker-Thurmond, K., \& Thun, M. J. (2003). Overweight, Obesity, and Mortality from Cancer in a Prospectively Studied Cohort of U.S. Adults. New England Journal of Medicine, 348, 1625-1638. http://doi.org/10.1056/NEJMoa021423 
Carlson, R. W., Allred, D. C., Anderson, B. O., Burstein, H. J., Carter, W. B., Edge, S. B., ... Zellars, R. (2011). Invasive Breast Cancer. Journal of the National Comprehensive Cancer Network, 9, 136-222.

Carver, C. S. (1997). You want to measure coping but your protocol's too long: Consider the brief cope. International journal of behavioral medicine, 4, 92-100.

Carver, C. S., Scheier, M. F., \& Weintraub, J. K. (1989). Assessing coping strategies: a theoretically based approach. Journal of personality and social psychology, 56, 267.

Cash, E., Sephton, S. E., Chagpar, A. B., Spiegel, D., Rebholz, W. N., Zimmaro, L. A., ... \& Dhabhar, F. S. (2015). Circadian disruption and biomarkers of tumor progression in breast cancer patients awaiting surgery. Brain, behavior, and immunity, 48, 102-114.

Caspi, A., Sugden, K., Moffitt, T. E., Taylor, A., Craig, I. W., Harrington, H., ... Poulton, R. (2003). Influence of Life Stress on Depression: Moderation by a Polymorphism in the 5-HTT Gene. Science, 301, 386-389. http://doi.org/10.1126/science. 1083968

Chang, C. M., Su, Y. C., Lai, N. S., Huang, K. Y., Chien, S. H., Chang, Y. H., ... Lee, C. C. (2012). The Combined Effect of Individual and Neighborhood Socioeconomic Status on Cancer Survival Rates. PLOS ONE, 7, e44325. http://doi.org/10.1371/journal.pone.0044325

Chan, S., \& Debono, M. (2010). Replication of cortisol circadian rhythm: new advances in hydrocortisone replacement therapy. Therapeutic Advances in Endocrinology and Metabolism, 1, 129-138. http://doi.org/10.1177/2042018810380214 
Chen, X., Wang, R., Zee, P., Lutsey, P. L., Javaheri, S., Alcántara, C., ... Redline, S. (2015). Racial/Ethnic Differences in Sleep Disturbances: The Multi-Ethnic Study of Atherosclerosis (MESA). Sleep, 38, 877-888.

http://doi.org/10.5665/sleep.4732

Chlebowski, R. T., Chen, Z., Anderson, G. L., Rohan, T., Aragaki, A., Lane, D., ... Prentice, R. (2005). Ethnicity and Breast Cancer: Factors Influencing Differences in Incidence and Outcome. Journal of the National Cancer Institute, 97, 439-448. http://doi.org/10.1093/jnci/dji064

Cimprich, B. (1999). Pretreatment symptom distress in women newly diagnosed with breast cancer. Cancer Nursing, 22, 185-194.

Clark, R., Anderson, N. B., Clark, V. R., \& Williams, D. R. (1999). Racism as a Stressor for African Americans: A Biopsychosocial Model. American Psychologist October 1999, 54, 805-816.

Cleeland, C. S., Bennett, G. J., Dantzer, R., Dougherty, P. M., Dunn, A. J., Meyers, C. A., ... Lee, B. N. (2003). Are the symptoms of cancer and cancer treatment due to a shared biologic mechanism? A cytokine-immunologic model of cancer symptoms. Cancer, 97, 2919-25. http://doi.org/10.1002/cncr.11382

Cohen, L., Cole, S. W., Sood, A. K., Prinsloo, S., Kirschbaum, C., Arevalo, J. M., ... Pisters, L. (2012). Depressive symptoms and cortisol rhythmicity predict survival in patients with renal cell carcinoma: role of inflammatory signaling. PLoS One, 7, e42324. http://doi.org/10.1371/journal.pone.0042324

Cohen, S., Schwartz, J. E., Epel, E., Kirschbaum, C., Sidney, S., \& Seeman, T. (2006). Socioeconomic Status, Race, and Diurnal Cortisol Decline in the Coronary Artery 
Risk Development in Young Adults (CARDIA) Study. Psychosomatic Medicine, 68, 41-50. http://doi.org/10.1097/01.psy.0000195967.51768.ea

Compas, B. E., \& Luecken, L. (2002). Psychological adjustment to breast cancer. Current Directions in Psychological Science, 11, 111-114.

Contrada, R. J., Ashmore, R. D., Gary, M. L., Coups, E., Egeth, J. D., Sewell, A., ... Chasse, V. (2000). Ethnicity-Related Sources of Stress and Their Effects on WellBeing. Current Directions in Psychological Science, 9, 136-139. http://doi.org/10.1111/1467-8721.00078

Contrada, R. J., Ashmore, R. D., Gary, M. L., Coups, E., Egeth, J. D., Sewell, A., ... Chasse, V. (2001). Measures of Ethnicity-Related Stress: Psychometric Properties, Ethnic Group Differences, and Associations With Well-Being1. Journal of Applied Social Psychology, 31, 1775-1820.

http://doi.org/10.1111/j.1559-1816.2001.tb00205.x

Cordova, M. J., Andrykowski, M. A., Kenady, D. E., McGrath, P. C., Sloan, D. A., \& Redd, W. H. (1995). Frequency and correlates of posttraumatic-stress-disorderlike symptoms after treatment for breast cancer. Journal of Consulting and Clinical Psychology, 63, 981-6.

Coulon, S. M., Wilson, D. K., Horn, M. L. V., Hand, G. A., \& Kresovich, S. (2015). The Association of Neighborhood Gene-Environment Susceptibility with Cortisol and Blood Pressure in African-American Adults. Annals of Behavioral Medicine, 50, 98-107. http://doi.org/10.1007/s12160-015-9737-9

Cozier, Y. C., Albert, M. A., Castro-Webb, N., Coogan, P. F., Ridker, P., Kaufman, H. W., ... Rosenberg, L. (2016). Neighborhood Socioeconomic Status in Relation to 
Serum Biomarkers in the Black Women's Health Study. Journal of Urban Health, 93, 279-291. http://doi.org/10.1007/s11524-016-0034-0

Culver, J. L., Arena, P. L., Antoni, M. H., \& Carver, C. S. (2002). Coping and distress among women under treatment for early stage breast cancer: comparing African Americans, Hispanics and non-Hispanic Whites. Psychooncology, 11, 495-504. http://doi.org/10.1002/pon.615

Davis, S., Mirick, D. K., \& Stevens, R. G. (2001). Night Shift Work, Light at Night, and Risk of Breast Cancer. Journal of the National Cancer Institute, 93, 1557-1562. http://doi.org/10.1093/jnci/93.20.1557

Dedert, E., Lush, E., Chagpar, A., Dhabhar, F. S., Segerstrom, S. C., Spiegel, D., ... Sephton, S. E. (2012). Stress, coping, and circadian disruption among women awaiting breast cancer surgery. Annals of Behavioral Medicine, 44, 10-20. http://doi.org/10.1007/s12160-012-9352-y

DeGuzman, P. B., \& Schminkey, D. L. (2016). Influencing Genomic Change and Cancer Disparities through Neighborhood Chronic Toxic Stress Exposure: A Research Framework. Public Health Nursing. http://doi.org/10.1111/phn.12290

Derynck, R., Akhurst, R. J., \& Balmain, A. (2001). TGF- $\beta$ signaling in tumor suppression and cancer progression. Nature Genetics, 29, 117-129. http://doi.org/10.1038/ng1001-117

Deryugina, E. I., \& Quigley, J. P. (2006). Matrix metalloproteinases and tumor metastasis. Cancer and Metastasis Reviews, 25, 9-34. http://doi.org/10.1007/s10555-006-7886-9 
DeSantis, A. S., Adam, E. K., Doane, L. D., Mineka, S., Zinbarg, R. E., \& Craske, M. G. (2007). Racial/Ethnic Differences in Cortisol Diurnal Rhythms in a Community Sample of Adolescents. Journal of Adolescent Health, 41, 3-13. http://doi.org/10.1016/j.jadohealth.2007.03.006

DeSantis, A. S., Adam, E. K., Hawkley, L. C., Kudielka, B. M., \& Cacioppo, J. T. (2015). Racial and ethnic differences in diurnal cortisol rhythms: are they consistent over time?. Psychosomatic medicine, 77, 6-15.

DeSantis, C., Ma, J., Bryan, L., \& Jemal, A. (2014). Breast cancer statistics, 2013. CA: A Cancer Journal for Clinicians, 64, 52-62. http://doi.org/10.3322/caac.21203

DeSantis, C., Siegel, R., Bandi, P., \& Jemal, A. (2011). Breast cancer statistics, 2011. CA: A Cancer Journal for Clinicians, 61, 408-418. http://doi.org/10.3322/caac.20134

Dettenborn, L., James, G. D., Valdimarsdottir, H. B., Montgomery, G. H., \& Bovbjerg, D. H. (2006). Breast cancer-specific intrusions are associated with increased cortisol responses to daily life stressors in healthy women without personal or family histories of breast cancer. Journal of Behavioral Medicine, 29, 477-485. http://doi.org/10.1007/s10865-006-9070-7

Diehr, P., Yergan, J., Chu, J., Feigl, P., Glaefke, G., Moe, R., ... Rodenbaugh, J. (1989). Treatment Modality and Quality Differences for Black and White Breast-Cancer Patients Treated in Community Hospitals. Medical Care, 27, 942-958.

Do, D. P., Diez Roux, A. V., Hajat, A., Auchincloss, A. H., Merkin, S. S., Ranjit, N., ... Seeman, T. (2011). Circadian rhythm of cortisol and neighborhood characteristics 
in a population-based sample: The Multi-Ethnic Study of Atherosclerosis. Health \& Place, 17, 625-632. http://doi.org/10.1016/j.healthplace.2010.12.019

Dulin-Keita, A., Casazza, K., Fernandez, J. R., Goran, M. I., \& Gower, B. (2012). Do neighbourhoods matter? Neighbourhood disorder and long-term trends in serum cortisol levels. Journal of Epidemiology and Community Health, 66, 24-29. http://doi.org/10.1136/jech.2009.092676

Dulin-Keita, A., Judd, S. E., Howard, V. J., Carson, A. P., Ard, J. D., \& Fernandez, J. R. (2014). Associations of neighborhood area level deprivation with the metabolic syndrome and inflammation among middle-and older-age adults. BMC public health, 14, 1 .

Du, X. L., Lin, C. C., Johnson, N. J., \& Altekruse, S. (2011). Effects of individual-level socioeconomic factors on racial disparities in cancer treatment and survival. Cancer, 117, 3242-3251. http://doi.org/10.1002/cncr.25854

Eismann, E. A., Lush, E., \& Sephton, S. E. (2010). Circadian effects in cancer-relevant psychoneuroendocrine and immune pathways. Psychoneuroendocrinology, 37, 963-976. http://doi.org/10.1016/j.psyneuen.2009.12.011

Elenkov, I. J., \& Chrousos, G. P. (1999). Stress Hormones, Th1/Th2 patterns, Pro/Antiinflammatory Cytokines and Susceptibility to Disease. Trends in Endocrinology \& Metabolism, 10, 359-368. http://doi.org/10.1016/S1043-2760(99)00188-5

Ellen, I. G., Mijanovich, T., \& Dillman, K.-N. (2001). Neighborhood Effects on Health: Exploring the Links and Assessing the Evidence. Journal of Urban Affairs, 23, 391-408. http://doi.org/10.1111/0735-2166.00096 
Epping-Jordan, J. E., Compas, B. E., \& Howell, D. C. (1994). Predictors of cancer progression in young adult men and women: Avoidance, intrusive thoughts, and psychological symptoms. Health Psychology, 13, 539-547. http://doi.org/10.1037/0278-6133.13.6.539

Faul, F., Erdfelder, E., Lang, A. G., \& Buchner, A. (2007). G*Power 3: A flexible statistical power analysis program for the social, behavioral, and biomedical sciences. Behavior Research Methods, 39, 175-191.

Fidler, I. J. (2003). The pathogenesis of cancer metastasis: the "seed and soil" hypothesis revisited. Nature Reviews Cancer, 3, 453-458. http://doi.org/10.1038/nrc1098

Field, A. (2009). Discovering Statistics Using SPSS. SAGE Publications.

Filipski, E., King, V. M., Li, X., Granda, T. G., Mormont, M. C., Liu, X., ... Lévi, F. (2002). Host Circadian Clock as a Control Point in Tumor Progression. Journal of the National Cancer Institute, 94, 690-697. http://doi.org/10.1093/jnci/94.9.690

Filipski, E., Li, X. M., \& Levi, F. (2006). Disruption of circadian coordination and malignant growth. Cancer Causes \& Control, 17, 509-14.

Filipski, E., Subramanian, P., Carriere, J., Guettier, C., Barbason, H., \& Levi, F. (2009). Circadian disruption accelerates liver carcinogenesis in mice. Mutation Research, 680, 95-105. http://doi.org/10.1016/j.mrgentox.2009.10.002

Fujimoto, H., Sangai, T., Ishii, G., Ikehara, A., Nagashima, T., Miyazaki, M., \& Ochiai, A. (2009). Stromal MCP-1 in mammary tumors induces tumor-associated macrophage infiltration and contributes to tumor progression. International Journal of Cancer, 125, 1276-1284. http://doi.org/10.1002/ijc.24378 
Gallagher, J., Parle, M., Cairns, D. (2002). Appraisal and psychological distress six months after diagnosis of breast cancer. British Journal of Health Psychology, 7, 365.

Gallagher-Thompson, D., Shurgot, G. R., Rider, K., Gray, H. L., McKibbin, C. L., Kraemer, H. C., ... Thompson, L. W. (2006). Ethnicity, Stress, and Cortisol Function in Hispanic and Non-Hispanic White Women: A Preliminary Study of Family Dementia Caregivers and Noncaregivers. The American Journal of Geriatric Psychiatry, 14, 334-342.

http://doi.org/10.1097/01.JGP.0000206485.73618.87

Gallo, L. C., Fortmann, A. L., de los Monteros, K. E., Mills, P. J., Barrett-Connor, E., Roesch, S. C., \& Matthews, K. A. (2012). Individual and Neighborhood Socioeconomic Status and Inflammation in Mexican-American Women: What is the Role of Obesity? Psychosomatic Medicine, 74, 535-542. http://doi.org/10.1097/PSY.0b013e31824f5f6d

Glynn, L. M., Schetter, C. D., Chicz-DeMet, A., Hobel, C. J., \& Sandman, C. A. (2007). Ethnic differences in adrenocorticotropic hormone, cortisol and corticotropinreleasing hormone during pregnancy. Peptides, 28, 1155-1161. http://doi.org/10.1016/j.peptides.2007.04.005

Grandner, M. A., Jackson, N. J., Izci-Balserak, B., Gallagher, R. A., Murray-Bachmann, R., Williams, N. J., ... Jean-Louis, G. (2015). Social and Behavioral Determinants of Perceived Insufficient Sleep. Frontiers in Neurology, 6, 112. http://doi.org/10.3389/fneur.2015.00112 
Greer, S., Morris, T., \& Pettingale, K. W. (1979). Psychological response to breast cancer: effect on outcome. The Lancet, 314, 785-787.

Grivennikov, S. I., Greten, F. R., \& Karin, M. (2010). Immunity, Inflammation, and Cancer. Cell, 140, 883-899. http://doi.org/10.1016/j.cell.2010.01.025

Grivennikov, S., \& Karin, M. (2008). Autocrine IL-6 Signaling: A Key Event in Tumorigenesis? Cancer Cell, 13, 7-9. http://doi.org/10.1016/j.ccr.2007.12.020

Guo, M., \& Hay, B. A. (1999). Cell proliferation and apoptosis. Current Opinion in Cell Biology, 11, 745-752. http://doi.org/10.1016/S0955-0674(99)00046-0

Haagsma, J. A., Polinder, S., Olff, M., Toet, H., Bonsel, G. J., \& van Beeck, E. F. (2012). Posttraumatic stress symptoms and health-related quality of life: a two year follow-up study of injury treated at the Emergency Department. BMC Psychiatry, 12, 1. http://doi.org/10.1186/1471-244X-12-1

Haas, J. S., Earle, C. C., Orav, J. E., Brawarsky, P., Keohane, M., Neville, B. A., \& Williams, D. R. (2008). Racial segregation and disparities in breast cancer care and mortality. Cancer, 113, 2166-2172. http://doi.org/10.1002/cncr.23828

Haden, S. T., Glowacki, J., Hurwitz, S., Rosen, C., \& LeBoff, M. S. (2000). Effects of age on serum dehydroepiandrosterone sulfate, IGF-I, and IL-6 levels in women. Calcified tissue international, 66, 414-418.

Hajat, A., Diez-Roux, A., Franklin, T. G., Seeman, T., Shrager, S., Ranjit, N., ... Kirschbaum, C. (2010). Socioeconomic and race/ethnic differences in daily salivary cortisol profiles: The Multi-Ethnic Study of Atherosclerosis. Psychoneuroendocrinology, 35, 932-943. http://doi.org/10.1016/j.psyneuen.2009.12.009 
Hajat, A., Moore, K., Do, D. P., Stein Merkin, S., Punjabi, N. M., Sáñchez, B. N., ... Diez-Roux, A. V. (2015). Examining the cross-sectional and longitudinal association between diurnal cortisol and neighborhood characteristics: Evidence from the multi-ethnic study of atherosclerosis. Health \& Place, 34, 199-206. http://doi.org/10.1016/j.healthplace.2015.05.017

Hale, L., \& Do, D. P. (2007). Racial Differences in Self-Reports of Sleep Duration in a Population-Based Study. Sleep, 30, 1096-1103.

Hale, L., Hill, T. D., \& Burdette, A. M. (2010). Does sleep quality mediate the association between neighborhood disorder and self-rated physical health? Preventive Medicine, 51, 275-278. http://doi.org/10.1016/j.ypmed.2010.06.017

Hamer, M., Chida, Y., \& Molloy, G. J. (2009). Psychological distress and cancer mortality. Journal of Psychosomatic Research, 66, 255-258. http://doi.org/10.1016/j.jpsychores.2008.11.002

Hanahan, D., \& Weinberg, R. A. (2011). Hallmarks of Cancer: The Next Generation. Cell, 144, 646-674. http://doi.org/10.1016/j.cell.2011.02.013

Hanson Frost, M., Suman, V. J., Rummans, T. A., Dose, A. M., Taylor, M., Novotny, P., ... Evans, R. E. (2000). Physical, psychological and social well-being of women with breast cancer: the influence of disease phase. Psycho-Oncology, 9, 221-231. http://doi.org/10.1002/1099-1611(200005/06)9:3<221::AID-PON456>3.0.CO;2$\mathrm{T}$

Hardeman, R. R., Perry, S. P., Phelan, S. M., Przedworski, J. M., Burgess, D. J., \& Ryn, M. van. (2016). Racial Identity and Mental Well-Being: The Experience of African American Medical Students, A Report from the Medical Student 
CHANGE Study. Journal of Racial and Ethnic Health Disparities, 3, 250-258. http://doi.org/10.1007/s40615-015-0136-5

Harrell, S. P. (2000). A Multidimensional Conceptualization of Racism-Related Stress: Implications for the Well-Being of People of Color. American Journal of Orthopsychiatry, 70, 42-57. http://doi.org/10.1037/h0087722

Harvey, A. G. (2002). Trouble in Bed: The Role of Pre-Sleep Worry and Intrusions in the Maintenance of Insomnia. Journal of Cognitive Psychotherapy, 16, 161-177. http://doi.org/10.1891/jcop.16.2.161.63992

Harvey, A. G., Tang, N. K. Y., \& Browning, L. (2005). Cognitive approaches to insomnia. Clinical Psychology Review, 25, 593-611. http://doi.org/10.1016/j.cpr.2005.04.005

Herichova, I. (2013). Changes of physiological functions induced by shift work. Endocrine Regulations, 47, 159-170. http://doi.org/10.4149/endo_2013_03_159 Hershman, D., McBride, R., Jacobson, J. S., Lamerato, L., Roberts, K., Grann, V. R., \& Neugut, A. I. (2005). Racial Disparities in Treatment and Survival Among Women With Early-Stage Breast Cancer. Journal of Clinical Oncology, 23, 6639-6646. http://doi.org/10.1200/JCO.2005.12.633

Hill, T. D., Burdette, A. M., \& Hale, L. (2009). Neighborhood disorder, sleep quality, and psychological distress: Testing a model of structural amplification. Health \& Place, 15, 1006-1013. http://doi.org/10.1016/j.healthplace.2009.04.001

Hill, T. D., Ross, C. E., \& Angel, R. J. (2005). Neighborhood Disorder, Psychophysiological Distress, and Health. Journal of Health and Social Behavior, 46, 170-186. http://doi.org/10.1177/002214650504600204 
Hodge, D. R., Hurt, E. M., \& Farrar, W. L. (2005). The role of IL-6 and STAT3 in inflammation and cancer. European Journal of Cancer, 41, 2502-2512. http://doi.org/10.1016/j.ejca.2005.08.016

Hoffmann, S. C., Stanley, E. M., Cox, E. D., DiMercurio, B. S., Koziol, D. E., Harlan, D. M., ... Blair, P. J. (2002). Ethnicity Greatly Influences Cytokine Gene Polymorphism Distribution. American Journal of Transplantation, 2, 560-567. http://doi.org/10.1034/j.1600-6143.2002.20611.x

Horowitz, M., Wilner, N., \& Alvarez, W. (1979). Impact of Event Scale: a measure of subjective stress. Psychosomatic Medicine, 41, 209-218.

Hoyt, M. A., Marin-Chollom, A. M., Bower, J. E., Thomas, K. S., Irwin, M. R., \& Stanton, A. L. (2014). Approach and avoidance coping: Diurnal cortisol rhythm in prostate cancer survivors. Psychoneuroendocrinology, 49, 182-186. http://doi.org/10.1016/j.psyneuen.2014.07.007

Husse, J., Eichele, G., \& Oster, H. (2015). Synchronization of the mammalian circadian timing system: Light can control peripheral clocks independently of the SCN clock. BioEssays, 37, 1119-1128. http://doi.org/10.1002/bies.201500026

Huynh, V. W., Guan, S. S. A., Almeida, D. M., McCreath, H., \& Fuligni, A. J. (2016). Everyday discrimination and diurnal cortisol during adolescence. Hormones and behavior, 80, 76-81.

Innominato, P. F., Focan, C., Gorlia, T., Moreau, T., Garufi, C., Waterhouse, J., ... Bjarnason, G. A. (2009). Circadian Rhythm in Rest and Activity: A Biological Correlate of Quality of Life and a Predictor of Survival in Patients with Metastatic 
Colorectal Cancer. Cancer Research, 69, 4700-4707. http://doi.org/10.1158/0008-5472.CAN-08-4747

Jackson, C. L., Redline, S., \& Emmons, K. M. (2015). Sleep as a Potential Fundamental Contributor to Disparities in Cardiovascular Health. Annual Review of Public Health, 36, 417-440. http://doi.org/10.1146/annurev-publhealth-031914-122838

Jacobs, L., \& Finlayson, C. (2010). Early Diagnosis and Treatment of Cancer Series: Breast Cancer: Expert Consult. Elsevier Health Sciences.

Jameson, D. W. (2003). Mind-Body Health and Stress Tolerance. Lincoln, NE: iUniverse.

Jargowsky, P. A., \& Yang, R. (2006). The "Underclass" Revisited: A Social Problem in Decline. Journal of Urban Affairs, 28, 55-70. http://doi.org/10.1111/j.07352166.2006.00259.x

Jean-Louis, G., Magai, M. C., Cohen, C.I., Zizi F., von Gizycki H., DiPalma J., Casimir G. J. (2001). Ethnic differences in self-reported sleep problems in older adults. Sleep, 24, 926-33.

Jean-Louis, G., Kripke, D. F., Ancoli-Israel, S., Klauber, M. R., \& Sepulveda, R. S. (2000). Sleep duration, illumination, and activity patterns in a population sample: effects of gender and ethnicity. Biological Psychiatry, 47, 921-927. http://doi.org/10.1016/S0006-3223(99)00169-9

Jin-no, K., Tanimizu, M., Hyodo, I., Nishikawa, Y., Hosokawa, Y., Doi, T., ... Okada, Y. (1998). Circulating vascular endothelial growth factor (VEGF) is a possible tumor marker for metastasis in human hepatocellular carcinoma. Journal of Gastroenterology, 33, 376-382. http://doi.org/10.1007/s005350050099 
Joseph, S. (2000). Psychometric Evaluation of Horowitz's Impact of Event Scale: A Review. Journal of Traumatic Stress, 13, 101-113. http://doi.org/10.1023/A:1007777032063

Karayiannakis, A. J., Syrigos, K. N., Polychronidis, A., Zbar, A., Kouraklis, G., Simopoulos, C., \& Karatzas, G. (2002). Circulating VEGF Levels in the Serum of Gastric Cancer Patients. Annals of Surgery, 236, 37-42.

Karb, R. A., Elliott, M. R., Dowd, J. B., \& Morenoff, J. D. (2012). Neighborhood-level stressors, social support, and diurnal patterns of cortisol: The Chicago Community Adult Health Study. Social Science \& Medicine, 75, 1038-1047. http://doi.org/10.1016/j.socscimed.2012.03.031

Kessler, R. C., Mickelson, K. D., \& Williams, D. R. (1999). The Prevalence, Distribution, and Mental Health Correlates of Perceived Discrimination in the United States. Journal of Health and Social Behavior, 40, 208. http://doi.org/10.2307/2676349

Kiecolt-Glaser, J. K., Preacher, K. J., MacCallum, R. C., Atkinson, C., Malarkey, W. B., \& Glaser, R. (2003). Chronic stress and age-related increases in the proinflammatory cytokine IL-6. Proceedings of the national Academy of Sciences, $100,9090-9095$.

Kim, H. J., \& Fredriksen-Goldsen, K. I. (2016). Disparities in Mental Health Quality of Life Between Hispanic and Non-Hispanic White LGB Midlife and Older Adults and the Influence of Lifetime Discrimination, Social Connectedness, Socioeconomic Status, and Perceived Stress. Research on Aging. http://doi.org/10.1177/0164027516650003 
Kim, Y., Shaffer, K. M., Rocha-Lima, C., Milton, A., \& Carver, C. S. (2014). Exploring the role of ethnicity on perceptions of cancer and physical health recovery during the first year of survivorship. Journal of health psychology, 21, 1331-1338.

Koj, A. (1974). Acute-Phase Reactants. In A. C. Allison (Ed.), Structure and Function of Plasma Proteins (pp. 73-131). New York, NY: Springer.

Komenaka, I. K., Martinez, M. E., Pennington, R. E., Hsu, C.H., Clare, S. E., Thompson, P. A., ... Goulet, R. J. (2010). Race and Ethnicity and Breast Cancer Outcomes in an Underinsured Population. Journal of the National Cancer Institute, 102, 1178 1187. http://doi.org/10.1093/jnci/djq215

Koopman, C., Butler, L. D., Classen, C., Giese-Davis, J., Morrow, G. R., Westendorf, J., ... Spiegel, D. (2002a). Traumatic stress symptoms among women with recently diagnosed primary breast cancer. Journal of Traumatic Stress, 15, 277-287. http://doi.org/10.1023/A:1016295610660

Koopman, C., Nouriani, B., Erickson, V., Anupindi, R., Butler, L. D., Bachmann, M. H., ... Spiegel, D. (2002b). Sleep Disturbances in Women With Metastatic Breast Cancer. The Breast Journal, 8, 362-370. http://doi.org/10.1046/j.15244741.2002.08606.x

Kraemer, H. C., Giese-Davis, J., Yutsis, M., O’Hara, R., Neri, E., Gallagher-Thompson, D., ... Spiegel, D. (2006). Design decisions to optimize reliability of daytime cortisol slopes in an older population. American Journal of Geriatric Psychiatry, $14,325-33$.

Kraemer, H. C., Kiernan, M., Essex, M., \& Kupfer, D. J. (2008). How and why criteria defining moderators and mediators differ between the Baron \& Kenny and 
MacArthur approaches. Health Psychology, 27(2, Suppl), S101-S108. http://doi.org/10.1037/0278-6133.27.2(Suppl.).S101

Kraemer, H. C., Stice, E., Kazdin, A., Offord, D., \& Kupfer, D. (2001). How do risk factors work together? Mediators, moderators, and independent, overlapping, and proxy risk factors. American Journal of Psychiatry, 158, 848-56.

Kronfol, Z., Nair, M., Zhang, Q., Hill, E. E., \& Brown, M. B. (1997). Circadian immune measures in healthy volunteers: relationship to hypothalamic-pituitary-adrenal axis hormones and sympathetic neurotransmitters. Psychosomatic Medicine, 59, $42-50$.

Kudielka, B. M., \& Wüst, S. (2010). Human models in acute and chronic stress: Assessing determinants of individual hypothalamus-pituitary-adrenal axis activity and reactivity. Stress: The International Journal on the Biology of Stress, 13, 1-14. http://doi.org/10.3109/10253890902874913

Kumari, M., Badrick, E., Chandola, T., Adler, N. E., Epel, E., Seeman, T., ... Marmot, M. (2010). Measures of Social Position and Cortisol Secretion in an Aging Population: Findings From the Whitehall II Study. Psychosomatic Medicine, 72, $27-34$.

Kwakkenbos, L., Coyne, J. C., \& Thombs, B. D. (2014). Prevalence of posttraumatic stress disorder (PTSD) in women with breast cancer. Journal of Psychosomatic Research, 76, 485-486. http://doi.org/10.1016/j.jpsychores.2014.03.103

Lackey, N. R., Gates, M. F., \& Brown, G. (2001). African American women's experiences with the initial discovery, diagnosis, and treatment of breast cancer. Oncology Nursing Forum, 28, 519-527. 
Lazarus, R., \& Folkman, S. (1984). Stress, Appraisal, and Coping. New York, NY: Springer.

Lemola, S., Ledermann, T., \& Friedman, E. M. (2013). Variability of Sleep Duration Is Related to Subjective Sleep Quality and Subjective Well-Being: An Actigraphy Study. PLOS ONE, 8, e71292. http://doi.org/10.1371/journal.pone.0071292

Lévi, F., Dugué, P. A., Innominato, P., Karaboué, A., Dispersyn, G., Parganiha, A., ... Spiegel, D. (2014). Wrist actimetry circadian rhythm as a robust predictor of colorectal cancer patients survival. Chronobiology International, 31(8), 891-900. http://doi.org/10.3109/07420528.2014.924523

Lichter, D. T., Parisi, D., \& Taquino, M. C. (2012). The Geography of Exclusion: Race, Segregation, and Concentrated Poverty. Social Problems, 59, 364-388. http://doi.org/10.1525/sp.2012.59.3.364

Li, C. I., Malone, K. E., \& Daling, J. R. (2003). Differences in breast cancer stage, treatment, and survival by race and ethnicity. Archives of internal medicine, 163, 49-56.

Li, J. C., \& Xu, F. (1997). Influences of Light-Dark Shifting on the Immune System, Tumor Growth and Life Span of Rats, Mice and Fruit Flies as well as on the Counteraction of Melatonin. Neurosignals, 6, 77-89. http://doi.org/10.1159/000109112

Lillie-Blanton, M., \& Laveist, T. (1996). Race/ethnicity, the social environment, and health. Social Science \& Medicine, 43, 83-91. http://doi.org/10.1016/02779536(95)00337-1 
Lutgendorf, S. K., Cole, S., Costanzo, E., Bradley, S., Coffin, J., Jabbari, S., ... Sood, A. K. (2003). Stress-Related Mediators Stimulate Vascular Endothelial Growth Factor Secretion by Two Ovarian Cancer Cell Lines. Clinical Cancer Research, 9, 4514-4521.

Lutgendorf, S. K., \& Costanzo, E. S. (2003). Psychoneuroimmunology and health psychology: an integrative model. Brain, Behavior, Immununity, 17, 225-232.

Lutgendorf, S. K., Johnsen, E. L., Cooper, B., Anderson, B., Sorosky, J. I., Buller, R. E., \& Sood, A. K. (2002). Vascular endothelial growth factor and social support in patients with ovarian carcinoma. Cancer, 95, 808-815.

Lutgendorf, Sood, A. K., \& Antoni, M. H. (2010). Host Factors and Cancer Progression: Biobehavioral Signaling Pathways and Interventions. Journal of Clinical Oncology, 28, 4094-4099. http://doi.org/10.1200/JCO.2009.26.9357

Lutgendorf, S. K., Weinrib, A. Z., Penedo, F., Russell, D., DeGeest, K., Costanzo, E. S., ... \& Cole, S. (2008). Interleukin-6, cortisol, and depressive symptoms in ovarian cancer patients. Journal of Clinical Oncology, 26, 4820-4827.

Mantovani, A., Allavena, P., Sica, A., \& Balkwill, F. (2008). Cancer-related inflammation. Nature, 454, 436-444. http://doi.org/10.1038/nature07205

Masi, C. M., Rickett, E. M., Hawkley, L. C., \& Cacioppo, J. T. (2004). Gender and ethnic differences in urinary stress hormones: the population-based Chicago Health, Aging, and Social Relations Study. Journal of Applied Physiology, 97, 941-947. http://doi.org/10.1152/japplphysiol.00256.2004 
Matthews, S. A., \& Yang, T. C. (2010). Exploring the Role of the Built and Social Neighborhood Environment in Moderating Stress and Health. Annals of Behavioral Medicine, 39, 170-183. http://doi.org/10.1007/s12160-010-9175-7

Mayberry, R. M., Mili, F., \& Ofili, E. (2000). Racial and Ethnic Differences in Access to Medical Care. Medical Care Research and Review, 57(4 suppl), 108-145. http://doi.org/10.1177/1077558700574006

Mayr, F. B., Spiel, A. O., Leitner, J. M., Firbas, C., Kliegel, T., \& Jilma, B. (2007). Ethnic differences in plasma levels of interleukin-8 (IL-8) and granulocyte colony stimulating factor (G-CSF). Translational Research, 149, 10-14. http://doi.org/10.1016/j.trsl.2006.06.003

Mcbride, C. M., Clipp, E., Peterson, B. L., Lipkus, I. M., \& Demark-Wahnefried, W. (2000). Psychological impact of diagnosis and risk reduction among cancer survivors. Psycho-Oncology, 9, 418-427. http://doi.org/10.1002/10991611(200009/10)9:5<418::AID-PON474>3.0.CO;2-E

McCallum, T. J., Sorocco, K. H., \& Fritsch, T. (2006). Mental Health and Diurnal Salivary Cortisol Patterns Among African American and European American Female Dementia Family Caregivers. Journal of Geriatric Psychiatry August 2006, 14, 684-693. http://doi.org/10.1097/01.JGP.0000225109.85406.89

McEwen, B. S. (2006). Protective and damaging effects of stress mediators: central role of the brain. Dialogues in Clinical Neuroscience, 8, 367-381.

McEwen, B. S. (1998). Protective and damaging effects of stress mediators: allostasis and allostatic load. The New England Journal of Medicine, 338, 171-179. 
McEwen B. S., \& Stellar E. (1993). Stress and the individual: Mechanisms leading to disease. Archives of Internal Medicine, 153, 2093-2101.

http://doi.org/10.1001/archinte.1993.00410180039004

Mertz, B. G., Bistrup, P. E., Johansen, C., Dalton, S. O., Deltour, I., Kehlet, H., \& Kroman, N. (2012). Psychological distress among women with newly diagnosed breast cancer. European Journal of Oncology Nursing, 16, 439-443. http://doi.org/10.1016/j.ejon.2011.10.001

Middleton, L. P., Chen, V., Perkins, G. H., Pinn, V., \& Page, D. (2003). Histopathology of breast cancer among African-American women. Cancer, 97(S1), 253-257.

Miles, F. L., Pruitt, F. L., van Golen, K. L., \& Cooper, C. R. (2007). Stepping out of the flow: capillary extravasation in cancer metastasis. Clinical \& Experimental Metastasis, 25, 305-324. http://doi.org/10.1007/s10585-007-9098-2

Miller, A. H., Ancoli-Israel, S., Bower, J. E., Capuron, L., \& Irwin, M. R. (2008). Neuroendocrine-Immune Mechanisms of Behavioral Comorbidities in Patients With Cancer. Journal of Clinical Oncology, 26, 971-982. http://doi.org/10.1200/JCO.2007.10.7805

Miller, G. E., Chen, E., \& Zhou, E. S. (2007). If It Goes Up, Must It Come Down? Chronic Stress and the Hypothalamic-Pituitary-Adrenocortical Axis in Humans. Psychological Bulletin January 2007, 133, 25-45. http://doi.org/10.1037/00332909.133.1.25

Miller, W. R., \& Ingle, J. N. (2002). Endocrine Therapy in Breast Cancer. Boca Raton, FL: CRC Press. 
Mohawk, J. A., Green, C. B., \& Takahashi, J. S. (2012). Central and Peripheral Circadian Clocks in Mammals. Annual Review of Neuroscience, 35, 445-462. http://doi.org/10.1146/annurev-neuro-060909-153128

Moreno-Smith, M., Lutgendorf, S. K., \& Sood, A. K. (2010). Impact of stress on cancer metastasis. Future Oncology, 6, 1863-1881. http://doi.org/10.2217/fon.10.142

Mormont, M. C., Bogdan, A, Cormont, S, Touitou, Y, Levi, F. (2002). Cortisol Diurnal Variation in Blood and Saliva of Patients with Metastatic Colorectal Cancer: Relevance for Clinical Outcome. Anticancer Research, 22, 1245.

Mormont, M. C., \& Levi, F. (1997). Circadian-system alterations during cancer processes: A review. International Journal of Cancer, 70, 241-247.

Mormont, M. C., \& Waterhouse, J. (2002). Contribution of the rest-activity circadian rhythm to quality of life in cancer patients. Chronobiology International, 19, 31323.

Mormont, M. C., Waterhouse, J., Bleuzen, P., Giacchetti, S., Jami, A., Bogdan, A., ... Levi, F. (2000). Marked 24-h rest/activity rhythms are associated with better quality of life, better response, and longer survival in patients with metastatic colorectal cancer and good performance status. Clinical Cancer Research, 6, $3038-45$.

Mosmann, T. R., \& Sad, S. (1996). The expanding universe of T-cell subsets: Th1, Th2 and more. Immunology Today, 17, 138-146. http://doi.org/10.1016/0167$5699(96) 80606-2$ 
Mundy-Bosse, B. L., Thornton, L. M., Yang, H. C., Andersen, B. L., \& Carson, W. E. (2011). Psychological stress is associated with altered levels of myeloid-derived suppressor cells in breast cancer patients. Cellular immunology, 270, 80-87.

Myers, H. F. (2009). Ethnicity- and socio-economic status-related stresses in context: an integrative review and conceptual model. Journal of Behavioral Medicine, 32, 919. http://doi.org/10.1007/s10865-008-9181-4

Nakamura, H., Kawasaki, N., Hagiwara, M., Saito, M., Konaka, C., \& Kato, H. (2000). Cellular immunologic parameters related to age, gender, and stage in lung cancer patients. Lung cancer, 28, 139-145.

Nazmi, A., Roux, A. D., Ranjit, N., Seeman, T. E., \& Jenny, N. S. (2010). Crosssectional and longitudinal associations of neighborhood characteristics with inflammatory markers: findings from the multi-ethnic study of atherosclerosis. Health \& Place, 16, 1104-1112.

Ness, R. B., Haggerty, C. L., Harger, G., \& Ferrell, R. (2004). Differential Distribution of Allelic Variants in Cytokine Genes among African Americans and White Americans. American Journal of Epidemiology, 160, 1033-1038. http://doi.org/10.1093/aje/kwh325

Northouse, L. L. (1989). The impact of breast cancer on patients and husbands. Cancer Nursing, 12, 278-284.

Oertelt-Prigione, S. (2012). The influence of sex and gender on the immune response. Autoimmunity reviews, 11, A479-A485.

Onitilo, A. A., Engel, J. M., Greenlee, R. T., \& Mukesh, B. N. (2009). Breast Cancer Subtypes Based on ER/PR and Her2 Expression: Comparison of 
Clinicopathologic Features and Survival. Clinical Medicine \& Research, 7, 4-13. http://doi.org/10.3121/cmr.2008.825

Ooi, S. L., Martinez, M. E., \& Li, C. I. (2011). Disparities in breast cancer characteristics and outcomes by race/ethnicity. Breast Cancer Research and Treatment, 127, 729-738. http://doi.org/10.1007/s10549-010-1191-6

Palesh, O., Aldridge-Gerry, A., Zeitzer, J. M., Koopman, C., Neri, E., Giese-Davis, J., ... \& Spiegel, D. (2014). Actigraphy-measured sleep disruption as a predictor of survival among women with advanced breast cancer. Sleep, 37, 837.

Pandey, M., Mathew, A., Abraham, E. K., \& Rajan, B. (2004). Primary sarcoma of the breast. Journal of Surgical Oncology, 87, 121-125. http://doi.org/10.1002/jso.20110

Parajuli, D. N. (2015). Do I Have Cancer?: Signs, Symptoms, Diagnoses, and Treatments of Fifty Common Cancers. Bloomington, IN: Balboa Press.

Perkins, P., Cooksley, C. D., \& Cox, J. D. (1996). Breast cancer: Is ethnicity an independent prognostic factor for survival? Cancer, 78, 1241-1247. http://doi.org/10.1002/(SICI)1097-0142(19960915)78:6<1241::AIDCNCR11>3.0.CO;2-0

Peterson, C. E., Rauscher, G. H., Johnson, T. P., Kirschner, C. V., Barrett, R. E., Kim, S., ... Davis, F. G. (2014). The association between neighborhood socioeconomic status and ovarian cancer tumor characteristics. Cancer Causes \& Control: CCC, 25, 633-637. http://doi.org/10.1007/s10552-014-0357-7 
Phinney, J. S. (1992). The Multigroup Ethnic Identity Measure A New Scale for Use with Diverse Groups. Journal of Adolescent Research, 7, 156-176. http://doi.org/10.1177/074355489272003

Phinney, J. S., \& Ong, A. D. (2007). Conceptualization and Measurement of Ethnic Identity: Current Status and Future Directions. Journal of Counseling Psychology, 54, 271-281.

Pine, S. R., Mechanic, L. E., Enewold, L., Bowman, E. D., Ryan, B. M., Cote, M. L., ... Harris, C. C. (2016). Differential Serum Cytokine Levels and Risk of Lung Cancer Between African and European Americans. Cancer Epidemiology Biomarkers \& Prevention, 25, 488-497. http://doi.org/10.1158/1055-9965.EPI15-0378

Primo, K., Compas, B. E., Oppedisano, G., Howell, D. C., Epping-Jordan, J. E., \& Krag, D. N. (2000). Intrusive thoughts and avoidance in breast cancer: Individual differences and association with psychological distress. Psychology \& Health, 14, 1141-1153. http://doi.org/10.1080/08870440008407372

Profant, J., Ancoli-Israel, S., \& Dimsdale, J. E. (2002). Are there ethnic differences in sleep architecture? American Journal of Human Biology, 14, 321-326. http://doi.org/10.1002/ajhb.10032

Pruessner, J., Hellhammer, D., \& Kirschbaum, C. (1999). Burnout, perceived stress, and cortisol responses to awakening. Psychosomatic Medicine, 61, 197-204.

Rao, U., Poland, R. E., Lutchmansingh, P., Ott, G. E., McCracken, J. T., \& Lin, K. M. (1999). Relationship between ethnicity and sleep patterns in normal controls: 
implications for psychopathology and treatment. Journal of Psychiatric Research, 33, 419-426. http://doi.org/10.1016/S0022-3956(99)00019-9

Rebholz, W. N., Cash, E., Zimmaro, L. A., Bayley-Veloso, R., Phillips, K., Siwik, C., ... Sephton, S. E. (2016). Distress and quality of life in an ethnically diverse sample awaiting breast cancer surgery. Journal of Health Psychology. http://doi.org/10.1177/1359105316659916

Reiche, E. M., Morimoto, H. K., \& Nunes, S. M. (2005). Stress and depression-induced immune dysfunction: implications for the development and progression of cancer. International Review of Psychiatry, 17, 515-27. http://doi.org/10.1080/02646830500382102

Reiche, E. M. V., Nunes, S. O. V., \& Morimoto, H. K. (2004). Stress, depression, the immune system, and cancer. The Lancet Oncology, 5, 617-625. http://doi.org/10.1016/S1470-2045(04)01597-9

Ross, C. E., \& Mirowsky, J. (2001). Neighborhood Disadvantage, Disorder, and Health. Journal of Health and Social Behavior, 42, 258. http://doi.org/10.2307/3090214 Salgado, R., Junius, S., Benoy, I., Van Dam, P., Vermeulen, P., Van Marck, E., ... Dirix, L. Y. (2003). Circulating interleukin-6 predicts survival in patients with metastatic breast cancer. International Journal of Cancer, 103, 642-646. http://doi.org/10.1002/ijc. 10833

Samuel, L. J., Roth, D. L., Schwartz, B. S., Thorpe, R. J., \& Glass, T. A. (2016). Socioeconomic Status, Race/Ethnicity, and Diurnal Cortisol Trajectories in Middle-Aged and Older Adults. The Journals of Gerontology Series B: 
Psychological Sciences and Social Sciences.

http://doi.org/10.1093/geronb/gbw080

Sapolsky, R. M. (2004). Why Zebras Don't Get Ulcers: The Acclaimed Guide to Stress, Stress-Related Diseases, and Coping $\left(3^{r d} E d\right.$.). New York, NY: Henry Holt and Company.

Savard, J., Liu, L., Natarajan, L., Rissling, M. B., Neikrug, A. B., He, F., ... AncoliIsrael, S. (2009). Breast Cancer Patients have Progressively Impaired Sleep-Wake Activity Rhythms during Chemotherapy. Sleep, 32, 1155-1160.

Scambia, G., Testa, U., Benedetti Panici, P., Foti, E., Martucci, R., Gadducci, A., ... Mancuso, S. (1995). Prognostic significance of interleukin 6 serum levels in patients with ovarian cancer. British Journal of Cancer, 71, 354-356.

Schag, C. C., Heinrich, R. L., \& Ganz, P. A. (1984). Karnofsky performance status revisited: reliability, validity, and guidelines. Journal of Clinical Oncology, 2, $187-193$.

Schernhammer, E. S., Kroenke, C. H., Laden, F., \& Hankinson, S. E. (2006). Night work and risk of breast cancer. Epidemiology, 17, 108-111.

Schernhammer, E. S., Laden, F., Speizer, F. E., Willett, W. C., Hunter, D. J., Kawachi, I., \& Colditz, G. A. (2001). Rotating night shifts and risk of breast cancer in women participating in the nurses' health study. Journal of the National Cancer Institute, 93, 1563-8.

Schibler, U., Gotic, I., Saini, C., Gos, P., Curie, T., Emmenegger, Y., ... Franken, P. (2015). Clock-Talk: Interactions between Central and Peripheral Circadian 
Oscillators in Mammals. Cold Spring Harbor Symposia on Quantitative Biology, 80, 223-232. http://doi.org/10.1101/sqb.2015.80.027490

Schlotz, W. (2004). Perceived Work Overload and Chronic Worrying Predict WeekendWeekday Differences in the Cortisol Awakening Response. Psychosomatic Medicine, 66, 207-214. http://doi.org/10.1097/01.psy.0000116715.78238.56

Schrepf, A., Thaker, P. H., Goodheart, M. J., Bender, D., Slavich, G. M., Dahmoush, L., ... Lutgendorf, S. K. (2015). Diurnal cortisol and survival in epithelial ovarian cancer. Psychoneuroendocrinology, 53, 256-267. http://doi.org/10.1016/j.psyneuen.2015.01.010

Schulz, P., Kirschbaum, C., Prüßner, J., \& Hellhammer, D. (1998). Increased free cortisol secretion after awakening in chronically stressed individuals due to work overload. Stress Medicine, 14, 91-97. http://doi.org/10.1002/(SICI)10991700(199804)14:2<91::AID-SMI765>3.0.CO;2-S

Segerstrom, S. C. (2004). Psychological Stress and the Human Immune System: A MetaAnalytic Study of 30 Years of Inquiry. Psychological Stress and the Human Immune System: A Meta-Analytic Study of 30 Years of Inquiry., 130, 601-630.

Sellers, R. M., Caldwell, C. H., Schmeelk-Cone, K. H., \& Zimmerman, M. A. (2003). Racial Identity, Racial Discrimination, Perceived Stress, and Psychological Distress among African American Young Adults. Journal of Health and Social Behavior, 44, 302. http://doi.org/10.2307/1519781

Sephton, S. E., Dhabhar, F. S., Keuroghlian, A. S., Giese-Davis, J., McEwen, B. S., Ionan, A. C., \& Spiegel, D. (2009). Depression, cortisol, and suppressed cell- 
mediated immunity in metastatic breast cancer. Brain, behavior, and immunity, $23,1148-1155$.

Sephton, S. E., Lush, E., Dedert, E. A., Floyd, A. R., Rebholz, W. N., Dhabhar, F. S., ... Salmon, P. (2013). Diurnal cortisol rhythm as a predictor of lung cancer survival. Brain, Behavior, and Immunity, 30, Supplement, S163-S170.

http://doi.org/10.1016/j.bbi.2012.07.019

Sephton, S. E., Sapolsky, R. M., Kraemer, H. C., \& Spiegel, D. (2000). Diurnal cortisol rhythm as a predictor of breast cancer survival. Journal of the National Cancer Institute, 92, 994-1000.

Seruga, B., Zhang, H., Bernstein, L. J., \& Tannock, I. F. (2008). Cytokines and their relationship to the symptoms and outcome of cancer. Nature Reviews Cancer, 8 , 887-99. http://doi.org/10.1038/nrc2507

Shariff-Marco, S., Klassen, A., \& Bowie, J. (2010). Racial/Ethnic Differences in SelfReported Racism and Its Association With Cancer-Related Health Behaviors. American Journal of Public Health, 100, 364-374.

Shavers, V. L., \& Brown, M. L. (2002). Racial and Ethnic Disparities in the Receipt of Cancer Treatment. Journal of the National Cancer Institute, 94, 334-357. http://doi.org/10.1093/jnci/94.5.334

Siegel, R., Ma, J., Zou, Z., \& Jemal, A. (2014). Cancer statistics, 2014. CA: A Cancer Journal for Clinicians, 64, 9-29. http://doi.org/10.3322/caac.21208

Silver, J. K. (2015). Cancer prehabilitation and its role in improving health outcomes and reducing health care costs. Seminars in oncology nursing, 31, 13-30. 
Singletary, S. E., Allred, C., Ashley, P., Bassett, L. W., Berry, D., Bland, K. I., ... Greene, F. L. (2002). Revision of the American Joint Committee on Cancer Staging System for Breast Cancer. Journal of Clinical Oncology, 20, 3628-3636. http://doi.org/10.1200/JCO.2002.02.026

Smith, S. M., \& Vale, W. W. (2006). The role of the hypothalamic-pituitary-adrenal axis in neuroendocrine responses to stress. Dialogues in Clinical Neuroscience, 8 , $383-395$.

Sood, A. K., Bhatty, R., Kamat, A. A., Landen, C. N., Han, L., Thaker, P. H., ... Cole, S. W. (2006). Stress Hormone-Mediated Invasion of Ovarian Cancer Cells. Clinical Cancer Research, 12, 369-375. http://doi.org/10.1158/1078-0432.CCR-05-1698

Spiegel, D., \& Sephton, S. E. (2001). Psychoneuroimmune and endocrine pathways in cancer: Effects of stress and support. Seminars in Clinical Neuropsychiatry, 6, $252-265$.

Sporn, M. B. (1996). The war on cancer. Lancet, 347, 1377.

Stepnowsky, C. J., Moore, P. J., \& Dimsdale, J. E. (2003). Effect of Ethnicity on Sleep: Complexities for Epidemiologic Research. Sleep, 26, 329-332.

Steptoe, A., \& Feldman, P. J. (2001). Neighborhood problems as sources of chronic stress: Development of a measure of neighborhood problems, and associations with socioeconomic status and health. Annals of Behavioral Medicine, 23, 177185. http://doi.org/10.1207/S15324796ABM2303_5

Steptoe, A., O’Donnell, K., Marmot, M., \& Wardle, J. (2008). Positive affect, psychological well-being, and good sleep. Journal of Psychosomatic Research, 64, 409-415. http://doi.org/10.1016/j.jpsychores.2007.11.008 
Sternthal, M. J., Slopen, N., \& Williams, D. R. (2011). RACIAL DISPARITIES IN HEALTH. Du Bois Review: Social Science Research on Race, 8, 95-113. http://doi.org/10.1017/S1742058X11000087

Stowe, R. P., Peek, M. K., Cutchin, M. P., \& Goodwin, J. S. (2010). Plasma Cytokine Levels in a Population-Based Study: Relation to Age and Ethnicity. The Journals of Gerontology Series A: Biological Sciences and Medical Sciences, 65A, 429433. http://doi.org/10.1093/gerona/glp198

Straif, K., Baan, Robert, Grosse, Yann, Secretan, Beatrice, Ghissassi, Fatiha El, Bouvard, Veronique, Altieri, Andrea, Benbrahim-Tallaa, Lamia, Cogliano, Vincent. (2007). Carcinogenicity of shift-work, painting, and fire-fighting. Lancet Oncology, 8, 1065-1066.

Suglia, S. F., Staudenmayer, J., Cohen, S., Enlow, M. B., Rich-Edwards, J. W., \& Wright, R. J. (2010). Cumulative Stress and Cortisol Disruption Among Black and Hispanic Pregnant Women in an Urban Cohort. Psychological Trauma: Theory, Research, Practice, \& Policy December 2010, 2, 326-334. http://doi.org/10.1037/a0018953

Swartzman, S., Booth, J. N., Munro, A., \& Sani, F. (2016). Posttraumatic stress disorder after cancer diagnosis in adults: A meta-analysis. Depression and Anxiety. http://doi.org/10.1002/da.22542

Tammemagi, C. M., Neslund-Dudas, C., Simoff, M., \& Kvale, P. (2004). Smoking and lung cancer survival*: The role of comorbidity and treatment. Chest, 125, 27-37. http://doi.org/10.1378/chest.125.1.27 
Taylor, K. L., Lamdan, R. M., Siegel, J. E., Shelby, R., Moran-Klimi, K., \& Hrywna, M. (2003). Psychological Adjustment Among African American Breast Cancer Patients: One-Year Follow-Up Results of a Randomized Psychoeducational Group Intervention. Health Psychology, 22, 316-323.

Taylor, T. R., Huntley, E. D., Makambi, K., Sween, J., Adams-Campbell, L. L., Frederick, W., \& Mellman, T. A. (2012). Understanding sleep disturbances in African-American breast cancer survivors: a pilot study. Psycho-Oncology, 21, 896-902. http://doi.org/10.1002/pon.2000

Taylor, S. E., \& Stanton, A. L. (2007). Coping resources, coping processes, and mental health. Annual. Review of Clinical Psychology, 3, 377-401.

Touitou, Y., Lévi, F., Bogdan, A., Benavides, M., Bailleul, F., \& Misset, J. L. (1995). Rhythm alteration in patients with metastatic breast cancer and poor prognostic factors. Journal of Cancer Research and Clinical Oncology, 121, 181-188. http://doi.org/10.1007/BF01198101

Tsimopoulou, I., Pasquali, S., Howard, R., Desai, A., Gourevitch, D., Tolosa, I., \& Vohra, R. (2015). Psychological prehabilitation before cancer surgery: a systematic review. Annals of Surgical Oncology, 22, 4117-4123.

Utsey, S. O., Chae, M. H., Brown, C. F., \& Kelly, D. (2002). Effect of Ethnic Group Membership on Ethnic Identity, Race-Related Stress, and Quality of Life. Cultural Diversity \& Ethnic Minority Psychology, 8, 366-377.

Vachani, C., \& Millar, L. B. (2015, March 30). Biologic Therapy: The Basics | OncoLink. Retrieved from http://www.oncolink.org/cancer-treatment/biologictherapy/biologic-therapy-the-basics 
Valković, T., Lučin, K., Krstulja, M., Dobi-Babić, R., \& Jonjić, N. (1998). Expression of monocyte chemotactic protein-1 in human invasive ductal breast cancer. Pathology - Research and Practice, 194, 335-340. http://doi.org/10.1016/S03440338(98)80057-5

Van Goethem, M., Schelfout, K., Kersschot, E., Colpaert, C., Weyler, J., Verslegers, I., ... Parizel, P. M. (2006). Comparison of MRI features of different grades of DCIS and invasive carcinoma of the breast. Clinical Imaging, 30, 225. http://doi.org/10.1016/j.clinimag.2006.01.009

Veikkola, T., \& Alitalo, K. (1999). VEGFs, receptors and angiogenesis. Seminars in Cancer Biology, 9, 211-220. http://doi.org/10.1006/scbi.1998.0091

Vin-Raviv, N., Hillyer, G. C., Hershman, D. L., Galea, S., Leoce, N., Bovbjerg, D. H., ... Neugut, A. I. (2013). Racial disparities in posttraumatic stress after diagnosis of localized breast cancer: the BQUAL study. Journal of the National Cancer Institute, 105, 563-572. http://doi.org/10.1093/jnci/djt024

Voigt, V., Neufeld, F., Kaste, J., Bühner, M., Sckopke, P., Wuerstlein, R., ... Hermelink, K. (2016). Clinically assessed posttraumatic stress in patients with breast cancer during the first year after diagnosis in the prospective, longitudinal, controlled COGNICARES study. Psycho-Oncology. http://doi.org/10.1002/pon.4102

Von Ah, D., Kang, D. H., \& Carpenter, J. S. (2007). Stress, optimism, and social support: Impact on immune responses in breast cancer. Research in nursing \& health, 30, 72-83.

Vos, M. S., Putter, H., van Houwelingen, H. C., \& de Haes, H. C. (2008). Denial in lung cancer patients: a longitudinal study. Psycho-Oncology, 17, 1163-1171. 
Vucenik, I., \& Stains, J. P. (2012). Obesity and cancer risk: evidence, mechanisms, and recommendations. Annals of the New York Academy of Sciences, 1271, 37-43. http://doi.org/10.1111/j.1749-6632.2012.06750.x

Waitzman, N. J., \& Smith, K. R. (1998). Phantom of the area: poverty-area residence and mortality in the United States. American Journal of Public Health, 88, 973-976.

Wang, X., Sánchez, B. N., Golden, S. H., Shrager, S., Kirschbaum, C., Karlamangla, A. S., ... Diez Roux, A. V. (2014). Stability and predictors of change in salivary cortisol measures over six years: MESA. Psychoneuroendocrinology, 49, 310 320. http://doi.org/10.1016/j.psyneuen.2014.07.024

Watson, M., Greer, S., Blake, S., \& Shrapnell, K. (1984). Reaction to a diagnosis of breast cancer relationship between denial, delay and rates of psychological morbidity. Cancer, 53, 2008-2012.

Wei, J., Xu, H., Davies, J. L., \& Hemmings, G. P. (1992). Increase of plasma IL-6 concentration with age in healthy subjects. Life sciences, 51, 1953-1956.

Weinrib, A. Z., Sephton, S. E., DeGeest, K., Penedo, F., Bender, D., Zimmerman, B., ... Lutgendorf, S. K. (2010). Diurnal cortisol dysregulation, functional disability, and depression in women with ovarian cancer. Cancer, 116, 4410-4419. http://doi.org/10.1002/cncr.25299

Whinnery, J., Jackson, N., Rattanaumpawan, P., \& Grandner, M. A. (2014). Short and long sleep duration associated with race/ethnicity, sociodemographics, and socioeconomic position. Sleep, 37, 601-611. http://doi.org/10.5665/sleep.3508

Wicklow, A., \& Espie, C. A. (2000). Intrusive thoughts and their relationship to actigraphic measurement of sleep: towards a cognitive model of insomnia. 
Behaviour Research and Therapy, 38, 679-693. http://doi.org/10.1016/S00057967(99)00136-9

Winkleby, M., Cubbin, C., \& Ahn, D. (2006). Effect of Cross-Level Interaction Between Individual and Neighborhood Socioeconomic Status on Adult Mortality Rates. American Journal of Public Health, 96, 2145-2153.

http://doi.org/10.2105/AJPH.2004.060970

Wohlfarth, T. D., van den Brink, W., Winkel, F. W., \& ter Smitten, M. (2003). Screening for Posttraumatic Stress Disorder: an evaluation of two self-report scales among crime victims. Psychological Assessment, 15, 101-109.

Wright, C. E., Schnur, J. B., Montgomery, G. H., \& Bovbjerg, D. H. (2010).

Psychological Factors Associated with Poor Sleep Prior to Breast Surgery: An Exploratory Study. Behavioral Medicine, 36, 85-91.

http://doi.org/10.1080/08964280903521305

Yehuda, R. (2002). Post-traumatic stress disorder. The New England Journal of Medicine, 346, 108-114. http://doi.org/10.1056/NEJMra012941

Yu J. B., Gross. C. P., Wilson L. D., Smith B. D. (2009). NCI SEER public-use data: applications and limitations in oncology research. Oncology, 23, 288-95.

Zabaleta, J., Schneider, B. G., Ryckman, K., Hooper, P. F., Camargo, M. C., Piazuelo, M. B., ... Ochoa, A. C. (2008). Ethnic differences in cytokine gene polymorphisms: potential implications for cancer development. Cancer Immunology, Immunotherapy, 57, 107-114. http://doi.org/10.1007/s00262-007-0358-4 
Zamarron, B. F., \& Chen, W. (2011). Dual Roles of Immune Cells and Their Factors in Cancer Development and Progression. International Journal of Biological Sciences, 7(5), 651-658.

Zeiders, K. H., Hoyt, L. T., \& Adam, E. K. (2014). Associations between self-reported discrimination and diurnal cortisol rhythms among young adults: The moderating role of racial-ethnic minority status. Psychoneuroendocrinology, 50, 280-288.

Zein, N. N., Germer, J. J., El-Zayadi, A. R., \& Vidigal, P. G. (2004). Ethnic Differences in Polymorphisms of Tumor Necrosis Factor- $\alpha$, Interleukin-10, and Transforming Growth Factor - $\beta 1$ Genes in Patients with Chronic Hepatitis C Virus Infection. The American Journal of Tropical Medicine and Hygiene, 70, 434-437.

Zheng Q., Safina, A., Bakin, A. V. (2008). Role of high-molecular weight tropomyosins in TGF-beta-mediated control of cell motility. International Journal of Cancer. Journal International Du Cancer, 122, 78-90. 


\section{APPENDIX A}

Abbreviations: DCIS, ductal carcinoma in situ; TNM, tumor-node-metastasis; ER, estrogen receptor; PR, progesterone receptor; HER2, human epidermal growth factor receptor 2; QOL, quality of life; SES, socioeconomic status; NH, non-Hispanic; ASD, acute stress disorder; PTSD, post-traumatic stress disorder; DSM, Diagnostic and Statistical Manual of Mental Disorders; SCN, suprachiasmatic nucleus; HPA, hypothalamic-pituitary-adrenal; CAR, cortisol awakening response; VEGF, vascular endothelial growth factor; MMP, matrix metalloproteinase; TGF, transforming growth factor; IL-6R, interleukin-6 receptor; MCP, monocyte chemoattractant protein; IL, interleukin; TNF, tumor necrosis factor; IFN, interferon; REM, rapid eye movement; GCSF, granulocyte-colony stimulating factor; CRP, C-reactive protein; SEER, Surveillance, Epidemiology, and End Results Program; AUC, area under the curve; KCR, Kentucky Cancer Registry; IES, Impact of Events Scale; MEMS, medication event monitoring system; EIA, enzyme immunoassay; CV, coefficient of variation; EFA, exploratory factor analysis; POM-TMD, Profile of Mood States- Total Mood Disturbance; PCA, principal component analysis; KMO, Kaiser-Meyer-Olkin; MEIM, Multigroup Ethnic Identity Measure 


\section{APPENDIX B}

Ethnicity (as measured in this study)

What is your racial-ethnic background?

$\begin{array}{ll}\mathrm{O} & \text { Asian } \\ \mathrm{O} & \text { Black } \\ \mathrm{O} & \text { Hispanic } \\ \mathrm{O} & \text { Native American } \\ \mathrm{O} & \text { White/Caucasian } \\ \mathrm{O} & \text { Other }\end{array}$




\section{Measure of Cancer-Related Stress}

Impact of Events Scale (IES; Horowitz et al., 1979)

Below is a list of comments made by people after stressful life events. Please fill in each item, indicating how frequently the comments were true for you DURING THE CURRENT DAY regarding your diagnosis and treatment of BREAST CANCER. If they did not occur, please mark the "Not at All" column.

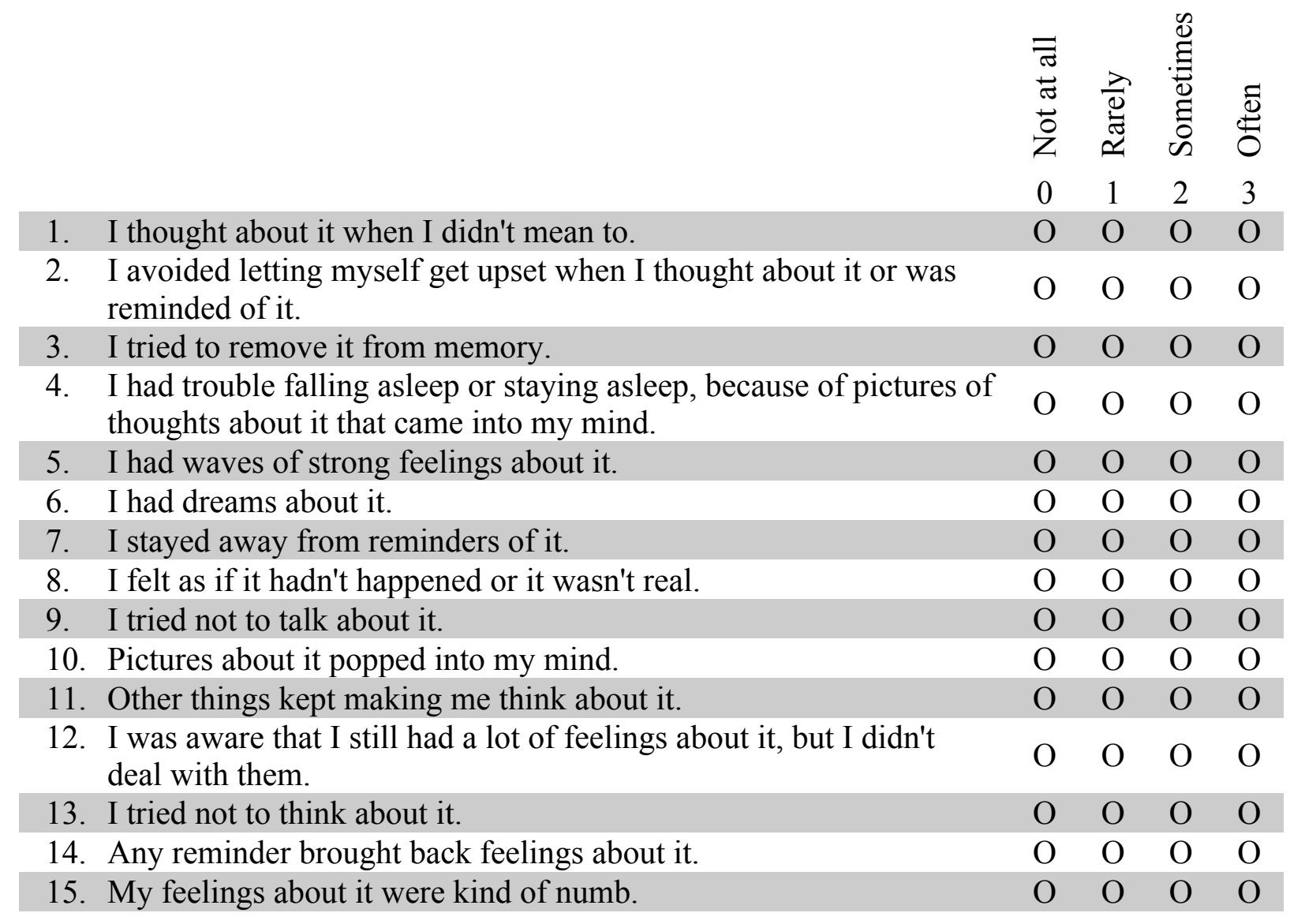




\section{APPENDIX C}

All results are presented here in full as a reference.

Spearman's rho correlation matrix for hypothesis 1.

\begin{tabular}{|c|c|c|c|c|}
\hline & Mean (SD) & 1 & 2 & 3 \\
\hline 1. Ethnicity & -- & -- & $.615^{*}$ & -.066 \\
\hline $\begin{array}{l}\text { 2. Neighborhood } \\
\text { socioeconomic } \\
\text { stress }\end{array}$ & $0(1)^{+}$ & -- & -- & .172 \\
\hline $\begin{array}{l}\text { 3. Cancer-related } \\
\text { stress }\end{array}$ & $28.38(15.37)$ & -- & -- & -- \\
\hline
\end{tabular}

Summary of results for hypothesis 2A.1: Hierarchical regression analyses entering ethnicity as a predictor of circadian rhythm variables.

\begin{tabular}{|c|c|c|c|c|c|c|c|}
\hline Variable & $\mathrm{N}$ & B & SE B & $\beta$ & $\mathrm{R}^{2}$ & $\Delta \mathrm{R}^{2}$ & p of $\Delta R^{2}$ \\
\hline Rest/activity rhythm & 43 & & & & & & \\
\hline Step 1 & & & & & .223 & .223 & $.019^{*}$ \\
\hline Age & & .003 & .002 & .209 & & & \\
\hline Stage & & .008 & .011 & .104 & & & \\
\hline Income & & .041 & .013 & $.436^{*}$ & & & \\
\hline Step 2 & & & & & .257 & .034 & .195 \\
\hline Ethnicity & & -.058 & .052 & -.170 & & & \\
\hline Nighttime restfulness & 43 & & & & & & \\
\hline Step 1 & & & & & .093 & .093 & 277 \\
\hline Age & & -.001 & .042 & -.005 & & & \\
\hline Stage & & .102 & .269 & .059 & & & \\
\hline Income & & .618 & .314 & .301 & & & \\
\hline Step 2 & & & & & .094 & .000 & .890 \\
\hline Ethnicity & & -.180 & 1.298 & -.024 & & & \\
\hline Daytime sedentariness & 43 & & & & & & \\
\hline Step 1 & & & & & .185 & .185 & $.045^{*}$ \\
\hline Age & & -.087 & .064 & -.200 & & & \\
\hline Stage & & .244 & 409 & .088 & & & \\
\hline Income & & -1.221 & 478 & $-.370 *$ & & & \\
\hline Step 2 & & & & & .189 & .004 & .655 \\
\hline Ethnicity & & -.890 & 1.974 & -.074 & & & \\
\hline
\end{tabular}

$* p<.05$ 
Summary of results for hypothesis 2A.1: Hierarchical regression analyses entering ethnicity as a predictor of endocrine disruption variables.

\begin{tabular}{|c|c|c|c|c|c|c|c|}
\hline Variable & $\mathrm{N}$ & B & SE B & $\beta$ & $\mathrm{R}^{2}$ & $\Delta \mathrm{R}^{2}$ & $p$ of $\Delta R^{2}$ \\
\hline CAR slope & 36 & & & & & & \\
\hline Step 1 & & & & & .041 & .041 & .714 \\
\hline Age & & -.006 & .007 & -.166 & & & \\
\hline Stage & & .013 & .044 & .051 & & & \\
\hline Income & & .019 & .053 & .061 & & & \\
\hline Step 2 & & & & & .119 & .078 & .107 \\
\hline Ethnicity & & .335 & .202 & .314 & & & \\
\hline Diurnal cortisol slope & 45 & & & & & & \\
\hline Step 1 & & & & & .056 & .056 & .495 \\
\hline Age & & -.001 & .001 & -.072 & & & \\
\hline Stage & & -.006 & .007 & -.133 & & & \\
\hline Income & & -.011 & .008 & -.201 & & & \\
\hline Step 2 & & & & & .056 & .000 & .908 \\
\hline Ethnicity & & .004 & .033 & .020 & & & \\
\hline $\begin{array}{l}\text { Diurnal cortisol mean } \\
\text { Step } 1\end{array}$ & 45 & & & & .070 & .070 & .391 \\
\hline Age & & .009 & .006 & .245 & & & \\
\hline Stage & & .035 & .038 & .143 & & & \\
\hline Income & & -.002 & .045 & -.005 & & & \\
\hline Step 2 & & & & & .081 & .011 & .491 \\
\hline Ethnicity & & .121 & .174 & .115 & & & \\
\hline
\end{tabular}

Summary of results for hypothesis 2A.1: Hierarchical regression analyses entering ethnicity as a predictor of immune factor variables.

\begin{tabular}{lccccccc}
\hline Variable & $\mathrm{N}$ & $\mathrm{B}$ & $\mathrm{SE} \mathrm{B}$ & $\beta$ & $\mathrm{R}^{2}$ & $\Delta \mathrm{R}^{2}$ & $\mathrm{p}$ of $\Delta \mathrm{R}^{2}$ \\
\hline Factor 1 & 36 & & & & .054 & .054 & .616 \\
Step 1 & & -.005 & .010 & -.078 & & & \\
Age & & -.088 & .071 & -.214 & & & \\
Stage & & -.056 & .078 & -.057 & & & \\
Income & & & & & .055 & .001 & .852 \\
Step 2 & & -.066 & .351 & -.039 & & & \\
Ethnicity & 36 & & & & & & \\
\hline Factor 2 & & & & & .07 & .076 & .465 \\
Step 1 & & .018 & .013 & .219 & & & \\
Age & & -.071 & .091 & -.132 & & & \\
Stage & & .071 & .101 & .120 & & & \\
Income & & -.767 & .432 & -.344 & & & .085 \\
Step 2 & & & & & & & \\
Ethnicity & 36 & & & & .342 & .342 & $.004^{*}$ \\
\hline Factor 3 & & & & & &
\end{tabular}




\begin{tabular}{lrrrrrrr} 
Age & -.045 & .011 & $-.560^{*}$ & & & \\
Stage & -.105 & .077 & -.194 & & & \\
Income & -.040 & .086 & -.067 & & & \\
Step 2 & & & & .388 & .046 & .137 \\
Ethnicity & .568 & .372 & .252 & & & \\
\hline
\end{tabular}

$* p<.05$

Summary of results for hypothesis 2A.2: Hierarchical regression analyses entering neighborhood socioeconomic stress as a predictor of circadian rhythm variables.

\begin{tabular}{|c|c|c|c|c|c|c|c|}
\hline Variable & $\mathrm{N}$ & B & SE B & $\beta$ & $\mathrm{R}^{2}$ & $\Delta \mathrm{R}^{2}$ & $\mathrm{p}$ of $\Delta \mathrm{R}^{2}$ \\
\hline Rest/activity rhythm & 40 & & & & & & \\
\hline Step 1 & & & & & .281 & .281 & $.007 *$ \\
\hline Age & & .003 & .002 & 214 & & & \\
\hline Stage & & .013 & .011 & .171 & & & \\
\hline Income & & .045 & .013 & $.497^{*}$ & & & \\
\hline Step 2 & & & & & .282 & .002 & .763 \\
\hline $\begin{array}{l}\text { Neighborhood } \\
\text { socioeconomic stress }\end{array}$ & & -.008 & .027 & -.048 & & & \\
\hline Nighttime restfulness & 40 & & & & & & \\
\hline Step 1 & & & & & .097 & .097 & .295 \\
\hline Age & & -.006 & .045 & -.023 & & & \\
\hline Stage & & .292 & .268 & .176 & & & \\
\hline Income & & .532 & .324 & .262 & & & \\
\hline Step 2 & & & & & .099 & .003 & .747 \\
\hline $\begin{array}{l}\text { Neighborhood } \\
\text { socioeconomic stress }\end{array}$ & & -.218 & .671 & -.058 & & & \\
\hline Daytime sedentariness & 40 & & & & & & \\
\hline Step 1 & & & & & .148 & .148 & .119 \\
\hline Age & & -.075 & .065 & -.180 & & & \\
\hline Stage & & -.202 & .389 & -.081 & & & \\
\hline Income & & -1.092 & 471 & $-.359 *$ & & & \\
\hline Step 2 & & & & & .163 & .014 & .444 \\
\hline $\begin{array}{l}\text { Neighborhood } \\
\text { socioeconomic stress }\end{array}$ & & .750 & .968 & .133 & & & \\
\hline
\end{tabular}

$* p<.05$

Summary of results for hypothesis 2A.2: Hierarchical regression analyses entering neighborhood socioeconomic stress as a predictor of endocrine disruption variables.

\begin{tabular}{lccccccc} 
Variable & $\mathrm{N}$ & $\mathrm{B}$ & $\mathrm{SE} \mathrm{B}$ & $\beta$ & $\mathrm{R}^{2}$ & $\Delta \mathrm{R}^{2}$ & $\mathrm{p}$ of $\Delta \mathrm{R}^{2}$ \\
\hline CAR slope & 34 & & & & & & \\
Step 1 & & & & & .046 & .046 & .696 \\
Age & & -.007 & .007 & -.183 & & & \\
Stage & & .005 & .043 & .023 & & & \\
Income & & .026 & .053 & .089 & & &
\end{tabular}


Step 2

$\begin{array}{llllll} & & & 080 & .034 & .308\end{array}$

Neighborhood

$\begin{array}{lll}-.141 & .136 & -.211\end{array}$

socioeconomic stress

Diurnal cortisol slope

Step 1

Age

41

Stage

Income

Step 2

Neighborhood

socioeconomic stress

Diurnal cortisol mean

Step 1

Age

Stage

Income

Step 2

Neighborhood

$\begin{array}{lll}-.001 & .001 \quad-.111\end{array}$

$.138 \quad .138$

.135

$\begin{array}{lll}-.001 & .006 & -.021\end{array}$

$-.018 \quad .007 \quad-.366^{*}$

$.139 \quad .001$

.829

socioeconomic stress

$* p<.05$

Summary of results for hypothesis 2A.2: Hierarchical regression analyses entering neighborhood socioeconomic stress as a predictor of immune factor variables.

\begin{tabular}{lccccccc}
\hline Variable & $\mathrm{N}$ & $\mathrm{B}$ & $\mathrm{SE} \mathrm{B}$ & $\beta$ & $\mathrm{R}^{2}$ & $\Delta \mathrm{R}^{2}$ & $\mathrm{p}$ of $\Delta \mathrm{R}^{2}$ \\
\hline Factor 1 & 34 & & & & & & \\
Step 1 & & & & & .076 & .076 & .490 \\
Age & & -.008 & .011 & -.126 & & & \\
Stage & & -.036 & .069 & -.091 & & & \\
Income & & -.114 & .080 & -.253 & & & \\
Step 2 & & & & & .180 & .103 & .066 \\
Neighborhood & & -.309 & .162 & -.356 & & & \\
socioeconomic stress & & & & & & & \\
\hline Factor 2 & 34 & & & & & & \\
Step 1 & & & & & .032 & .032 & .803 \\
Age & & .013 & .015 & .163 & & & \\
Stage & & -.017 & .088 & -.034 & & & \\
Income & & .048 & .102 & .086 & & & \\
Step 2 & & & & .049 & .017 & .477 \\
Neighborhood & & -.156 & .217 & -.145 & & & \\
Socioeconomic stress & & & & & & & \\
Factor 3 & 34 & & & & & & \\
Step 1 & & & & & .438 & .438 & $.001 *$ \\
Age & -.046 & .011 & $-.602^{*}$ & & & \\
Stage & -.150 & .064 & $-.324 *$ & & & \\
Income & -.070 & .074 & -.950 & & &
\end{tabular}


Step 2

$.473 \quad .035$

Neighborhood

$.213 \quad .154 \quad .207$

socioeconomic stress

$* p<.05$

Summary of results for hypothesis 2A.3: Hierarchical regression analyses entering cancer-related stress as a predictor of circadian rhythm variables.

\begin{tabular}{|c|c|c|c|c|c|c|c|}
\hline Variable & $\mathrm{N}$ & $\mathrm{B}$ & SE B & $\beta$ & $\mathrm{R}^{2}$ & $\Delta \mathrm{R}^{2}$ & $\mathrm{p}$ of $\Delta \mathrm{R}^{2}$ \\
\hline Rest/activity rhythm & 45 & & & & & & \\
\hline Step 1 & & & & & .236 & .236 & $.011 *$ \\
\hline Age & & .002 & .002 & .191 & & & \\
\hline Stage & & .010 & .011 & .127 & & & \\
\hline Income & & .041 & .012 & $.461 *$ & & & \\
\hline Step 2 & & & & & .176 & .015 & .374 \\
\hline Cancer-related stress & & -.001 & .001 & -.123 & & & \\
\hline Nighttime restfulness & 45 & & & & & & \\
\hline Step 1 & & & & & .092 & .092 & .262 \\
\hline Age & & .000 & .039 & .000 & & & \\
\hline Stage & & .246 & .250 & .149 & & & \\
\hline Income & & .523 & .291 & .269 & & & \\
\hline Step 2 & & & & & .092 & .000 & .972 \\
\hline Cancer-related stress & & .001 & .034 & .005 & & & \\
\hline Daytime sedentariness & 45 & & & & & & \\
\hline Step 1 & & & & & .156 & .156 & .071 \\
\hline Age & & -.074 & .058 & -.188 & & & \\
\hline Stage & & -.140 & .366 & -.056 & & & \\
\hline Income & & -1.087 & .426 & $-.368 *$ & & & \\
\hline Step 2 & & & & & .160 & .004 & .664 \\
\hline Cancer-related stress & & .022 & .050 & .064 & & & \\
\hline
\end{tabular}

$* p<.05$

Summary of results for hypothesis 2A.3: Hierarchical regression analyses entering cancer-related stress as a predictor of endocrine disruption variables.

\begin{tabular}{lccccccr}
\hline Variable & $\mathrm{N}$ & $\mathrm{B}$ & $\mathrm{SE} \mathrm{B}$ & $\beta$ & $\mathrm{R}^{2}$ & $\Delta \mathrm{R}^{2}$ & $\mathrm{p}$ of $\Delta \mathrm{R}^{2}$ \\
\hline CAR slope & 39 & & & & & & \\
Step 1 & & & & & .042 & .042 & .674 \\
Age & & -.006 & .006 & -.181 & & & \\
Stage & & .003 & .040 & .014 & & & \\
Income & & .019 & .049 & .067 & & & \\
Step 2 & & & & & .058 & .016 & .455 \\
Cancer-related stress & & -.004 & .005 & -.129 & & & \\
Diurnal cortisol slope & 47 & & & & & & \\
Step 1 & & & & & .071 & .071 & .358 \\
Age & & -.001 & .001 & -.099 & & &
\end{tabular}




\begin{tabular}{|c|c|c|c|c|c|c|c|}
\hline Stage & & -.002 & .006 & -.053 & & & \\
\hline Income & & -.013 & .008 & -.258 & & & \\
\hline Step 2 & & & & & .156 & .084 & $.047^{*}$ \\
\hline Cancer-related stress & & -.002 & .001 & -.292 & & & \\
\hline Diurnal cortisol mean & 47 & & & & & & \\
\hline Step 1 & & & & & .067 & .067 & .391 \\
\hline Age & & .008 & .006 & .227 & & & \\
\hline Stage & & .041 & .036 & .169 & & & \\
\hline Income & & .003 & .043 & .011 & & & \\
\hline Step 2 & & & & & .118 & .051 & .127 \\
\hline Cancer-related stress & & .007 & .005 & .227 & & & \\
\hline
\end{tabular}

$* p<.05$

Summary of results for hypothesis 2A.3: Hierarchical regression analyses entering cancer-related stress as a predictor of immune factor variables.

\begin{tabular}{|c|c|c|c|c|c|c|c|}
\hline Variable & $\mathrm{N}$ & $\mathrm{B}$ & SE B & $\beta$ & $\mathrm{R}^{2}$ & $\Delta \mathrm{R}^{2}$ & $\mathrm{p}$ of $\Delta \mathrm{R}^{2}$ \\
\hline Factor 1 & 39 & & & & & & \\
\hline Step 1 & & & & & .050 & .050 & .608 \\
\hline Age & & -.002 & .011 & -.037 & & & \\
\hline Stage & & -.060 & .069 & -.144 & & & \\
\hline Income & & -.085 & .079 & -.180 & & & \\
\hline Step 2 & & & & & .051 & .001 & .853 \\
\hline Cancer-related stress & & -.002 & .009 & -.031 & & & \\
\hline Factor 2 & 39 & & & & & & \\
\hline Step 1 & & & & & .064 & .064 & .502 \\
\hline Age & & .018 & .013 & .227 & & & \\
\hline Stage & & -.046 & .082 & -.092 & & & \\
\hline Income & & .040 & .094 & .070 & & & \\
\hline Step 2 & & & & & .074 & .010 & .551 \\
\hline Cancer-related stress & & .007 & .011 & .100 & & & \\
\hline Factor 3 & 39 & & & & & & \\
\hline Step 1 & & & & & .288 & .288 & $.007 *$ \\
\hline Age & & -.040 & .011 & $-.505^{*}$ & & & \\
\hline Stage & & -.116 & .073 & -.229 & & & \\
\hline Income & & -.069 & .083 & -.120 & & & \\
\hline Step 2 & & & & & .315 & .028 & .248 \\
\hline Cancer-related stress & & .011 & .009 & .168 & & & \\
\hline
\end{tabular}

$* p<.05$

Summary of results for hypothesis 2 B.1: Cox proportional hazards model entering ethnicity as a predictor of progression-free survival.

\begin{tabular}{|c|c|c|c|c|c|}
\hline 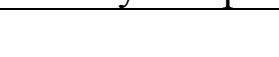 & $\mathrm{B}$ & SE B & Wald & Hazards Ratio & 95\% Confidence Interval \\
\hline 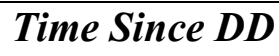 & & & & & \\
\hline Ethnicity & .157 & .579 & .073 & 1.170 & $.376-3.637$ \\
\hline
\end{tabular}


Time Since $\mathrm{SE}^{+}$

Ethnicity

$-.078 \quad .542 \quad .021$

.925

$.320-2.678$

$* p<.05$

${ }^{+}$Study entry (SE) calculated from date of first cortisol collection

Summary of results for hypothesis 2B.2: Cox proportional hazards model entering neighborhood socioeconomic stress as a predictor of progression-free survival.

\begin{tabular}{|c|c|c|c|c|c|}
\hline \multirow{2}{*}{ Time Since DD } & $\mathrm{B}$ & SE B & Wald & Hazards Ratio & $95 \%$ Confidence Interval \\
\hline & & & & & \\
\hline $\begin{array}{l}\text { Neighborhood } \\
\text { socioeconomic } \\
\text { stress }\end{array}$ & .302 & .263 & 1.319 & 1.352 & $.808-2.263$ \\
\hline Time Since $S E^{+}$ & & & & & \\
\hline $\begin{array}{l}\text { Neighborhood } \\
\text { socioeconomic } \\
\text { stress }\end{array}$ & .271 & .257 & 1.112 & 1.311 & $.793-2.168$ \\
\hline
\end{tabular}

Summary of results for hypothesis 2B.3: Cox proportional hazards model entering cancer-related stress as a predictor of progression-free survival.

\begin{tabular}{|c|c|c|c|c|c|}
\hline & $\mathrm{B}$ & SE B & Wald & Hazards Ratio & $95 \%$ Confidence Interval \\
\hline Time Since DD & & & & & \\
\hline $\begin{array}{l}\text { Cancer-related } \\
\text { stress }\end{array}$ & -.001 & .020 & .003 & .999 & $.961-1.038$ \\
\hline Time Since $S E^{+}$ & & & & & \\
\hline $\begin{array}{l}\text { Cancer-related } \\
\text { stress }\end{array}$ & -.014 & .019 & .569 & .986 & $.950-1.023$ \\
\hline
\end{tabular}

Summary of results for hypothesis 2C.1: Cox proportional hazards model entering circadian disruption variables as predictors of progression-free survival.

\begin{tabular}{lccccc}
\hline & B & SE B & Wald & Hazards Ratio & 95\% Confidence Interval \\
\hline $\begin{array}{l}\text { Time Since DD } \\
\begin{array}{l}\text { Rest/activity } \\
\text { rhythm }\end{array}\end{array}$ & .712 & 1.539 & .214 & 2.038 & $.100-41.633$ \\
$\begin{array}{l}\text { Nighttime } \\
\text { restfulness }\end{array}$ & -.012 & .082 & .880 & 1.012 & $.862-1.188$ \\
$\begin{array}{l}\text { Daytime } \\
\text { sedentariness }\end{array}$ & .000 & .050 & .000 & 1.000 & $.907-1.103$ \\
& & & & &
\end{tabular}




\section{Time Since $\mathrm{SE}^{+}$}

\begin{tabular}{|c|c|c|c|c|c|}
\hline $\begin{array}{l}\text { Rest/activity } \\
\text { rhythm }\end{array}$ & .910 & 1.524 & .356 & 2.484 & $.125-49.285$ \\
\hline $\begin{array}{l}\text { Nighttime } \\
\text { restfulness }\end{array}$ & -.016 & .082 & .040 & 1.016 & $.865-1.194$ \\
\hline $\begin{array}{l}\text { Daytime } \\
\text { sedentariness }\end{array}$ & -.002 & .050 & .001 & .998 & $.904-1.102$ \\
\hline
\end{tabular}

${ }^{+}$Study entry (SE) calculated from date of first actigraphy assessment

Summary of results for hypothesis 2C.2: Cox proportional hazards model entering endocrine disruption variables as predictors of progression-free survival.

\begin{tabular}{|c|c|c|c|c|c|}
\hline & $\mathrm{B}$ & SE B & Wald & Hazards Ratio & $95 \%$ Confidence Interval \\
\hline \multicolumn{6}{|l|}{ Time Since DD } \\
\hline CAR slope & .470 & .739 & .404 & 1.599 & $.376-6.806$ \\
\hline $\begin{array}{l}\text { Diurnal cortisol } \\
\text { slope }\end{array}$ & .093 & 3.166 & .001 & 1.098 & $.002-543.143$ \\
\hline $\begin{array}{l}\text { Diurnal cortisol } \\
\text { mean }\end{array}$ & .463 & .531 & .762 & 1.589 & $.562-4.496$ \\
\hline \multicolumn{6}{|l|}{ Time Since $S E^{+}$} \\
\hline CAR slope & .404 & .731 & .306 & 1.498 & $.357-6.281$ \\
\hline $\begin{array}{l}\text { Diurnal cortisol } \\
\text { slope }\end{array}$ & 1.207 & 2.926 & .170 & 3.343 & $.011-1035.063$ \\
\hline $\begin{array}{l}\text { Diurnal cortisol } \\
\text { mean }\end{array}$ & .421 & .498 & .716 & 1.524 & $.574-4.045$ \\
\hline
\end{tabular}

Summary of results for hypothesis 2C.3: Cox proportional hazards model entering immune factors as predictors of progression-free survival.

\begin{tabular}{lccccc}
\hline & B & SE B & Wald & Hazards Ratio & 95\% Confidence Interval \\
\hline Time Since DD & & & & & \\
Factor 1 & -.168 & .258 & .422 & .846 & $.510-1.402$ \\
Factor 2 & -.108 & .359 & .091 & .897 & $.444-1.812$ \\
Factor 3 & -.123 & .304 & .163 & .884 & $.487-1.606$ \\
\hline
\end{tabular}

\section{Time Since $\mathrm{SE}^{+}$}




\begin{tabular}{llllll} 
Factor 1 & -.168 & .251 & .448 & .846 & $.518-1.382$ \\
Factor 2 & -.226 & .338 & .447 & .798 & $.412-1.546$ \\
Factor 3 & -.214 & .291 & .542 & .807 & $.456-1.428$ \\
\hline
\end{tabular}

$* p<.05$

+ Study entry (SE) calculated from date of blood draw

Summary of results for post hoc analyses: Hierarchical regression analyses entered cancer-related stress subscales as a predictor of diurnal cortisol slope.

\begin{tabular}{lccccccr}
\hline Variable & $\mathrm{N}$ & $\mathrm{B}$ & $\mathrm{SE} \mathrm{B}$ & $\beta$ & $\mathrm{R}^{2}$ & $\Delta \mathrm{R}^{2}$ & $\mathrm{p}$ of $\Delta \mathrm{R}^{2}$ \\
\hline Diurnal Cortisol Slope & 48 & & & & & & \\
Step 1 & & & & & .091 & .091 & .234 \\
Age & & -.001 & .001 & -.124 & & & \\
Stage & -.003 & .006 & -.077 & & & \\
Income & -.015 & .008 & -.283 & & & \\
Step 2 & & & & .107 & .015 & .395 \\
Cancer-related stress: & -.001 & .002 & -.124 & & & \\
Intrusions & & & & & & \\
Step 2 & & & & .244 & .153 & $.005^{*}$ \\
Cancer-related stress: & -.004 & .001 & -.399 & & & \\
Avoidance & & & & & & & \\
\hline$* p<.05$ & & & & & &
\end{tabular}




\title{
CURRICULUM VITA
}

\author{
Whitney N. Rebholz, M.A. \\ 1603 Keever Court \\ Louisville, Kentucky 40245 \\ Phone: 502.432.6974 \\ e-mail: whitney.rebholz@gmail.com
}

\section{Education}

University of Louisville

Expected Graduation Date: December

2016

Doctor of Philosophy in Clinical Psychology

Current Cumulative GPA: 3.89

Dissertation title: Distress, Circadian Rhythms, Immunity, and Survival in

Presurgical Breast Cancer Patients

Mentor: Sandra Sephton, Ph.D.

University of Louisville

Master of Arts in Clinical Psychology

Graduated December 2013

Cumulative GPA: 3.86

University of Louisville

Graduated May 2011

Bachelor of Arts in Psychology, Concentration: Natural Sciences

Cum Laude with Psychology Honors

Cumulative GPA: 3.60

\section{Honors and Awards}

\begin{tabular}{|c|c|c|}
\hline 2014 & & $\begin{array}{l}\text { University of Louisville Clinical Psychology Excellence in } \\
\text { Peer Mentoring Award; chosen by peers for excellence in } \\
\text { peer mentoring }\end{array}$ \\
\hline 2014 & Spring & $\begin{array}{l}\text { American Psychosomatic Society (APS) Scholar } \\
\text { Award; } \$ 500 \text { award for an outstanding abstract } \\
\text { submission }\end{array}$ \\
\hline 2014 & Spring & $\begin{array}{l}\text { University of Louisville Arts and Sciences Graduate } \\
\text { Student Union Research Fund; } \$ 100 \text { award to assist } \\
\text { in travel to a conference to present research }\end{array}$ \\
\hline 2013 & Fall & $\begin{array}{l}\text { American Psychological Association of Graduate } \\
\text { Students Excellence in Campus Leadership Award; } \\
\text { presented to the highest point earner for the semester } \\
\text { in each region }\end{array}$ \\
\hline
\end{tabular}




\begin{tabular}{|c|c|}
\hline 2011 Spring & Dean's Scholar \\
\hline 2011 Spring & $\begin{array}{l}\text { Amber M. Heuerman Research Fund; } \$ 500 \text { award to } \\
\text { assist in presentation of research at a national conference. }\end{array}$ \\
\hline 2010 Fall & Dean's Scholar \\
\hline 2010 Spring & Dean's List \\
\hline 2009 Fall & Dean's Scholar \\
\hline 2008 Fall & Dean's List \\
\hline 2007-2011 & $\begin{array}{l}\text { Trustees' Scholarship: President's Scholar } \\
\text { This scholarship is a full-tuition (Kentucky in-state resident } \\
\text { rate) scholarship for one full year and is renewable for an } \\
\text { additional six semesters. }\end{array}$ \\
\hline 2007-2011 & $\begin{array}{l}\text { Kentucky Educational Excellence Scholarship } \\
\text { This scholarship awards } \$ 2,500 \text { each year for four years. }\end{array}$ \\
\hline esearch Experience & \\
\hline
\end{tabular}

Dissertation: Stress, Circadian Rhythms, Immune Function, and Tumor Progression in Presurgical Breast Cancer Patients

Among presurgical breast cancer patients the relationships between the unique stressors of ethnicity-related stress, neighborhood stress, and cancer-related stress will be explored. Additionally, the relationships between distress and stress and the relationships between distress, circadian rhythms, and immune function with cancer outcomes will be explored. Dissertation Chair/Mentor: Sandra Sephton, Ph.D.

Committee Members: Paul Salmon, Ph.D.; Barbara Stetson, Ph.D.; Tamara Newton, Ph.D.; Elizabeth Cash, Ph.D.

Project Director: Effects of a Meditation program on Illness Symptoms, Neuroendocrine and Immune Responses in Women with Fibromyalgia August 2013-January 2015

Manage regulatory issues through the Internal Review Board for this study on the effects of the Mindfulness-Based Stress Reduction (MBSR) program in women with fibromyalgia.

Directed by Sandra Sephton, Ph.D.

Project Director: Psychosocial Effects in Lung Cancer Outcomes July 2011-July 2015 Manage regulatory issues through the Internal Review Board for this study on the effects of psychosocial factors in the outcomes in lung cancer patients.

Directed by Sandra Sephton, Ph.D.

Project Director: Effects of Stress in Ovarian Cancer Outcomes July 2011-July 2015 Manage regulatory issues through the Internal Review Board for this study on the effects of stress in women with gynecological cancers.

Directed by Sandra Sephton, Ph.D.

Project Director: Stress, Coping, and Sleep in Breast Cancer

July 2011-Present 
Manage regulatory issues through the Internal Review Board for this study on the effects of stress, sleep, and cope in breast cancer patients.

Directed by Sandra Sephton, Ph.D.

Project Director: iPod-Based Coping Skills for Newly Diagnosed Breast Cancer Patients May 2011-July 2015

Manage regulatory issues for an iPod-based coping skills intervention in breast cancer patients. Credentialed through University of Louisville Hospital to recruit patients from the surgical oncology clinics. Prepare study materials to recruit participants. Meet with physicians and other personnel to prepare and continue effective recruitment. Recruit patients for a study on the effectiveness of iPod-based coping skills intervention. Meet with participants when necessary to deliver or collect study materials. Process salivary cortisol samples, blood samples, actigraphy, and logger data provided by patients enrolled in the study.

Directed by Sandra Sephton, Ph.D.

Undergraduate Honors' Thesis Project

August 2010-April 2011

An undergraduate honors thesis, "Distress and Immunity in Women Newly Diagnosed with Breast Cancer" was developed. This study analyzed the effects of immunity, measured via cytokine levels, on cancer-specific distress in women newly diagnosed with breast cancer. A thesis proposal was written and was approved on September 22, 2010. Honors thesis was orally defended and approved by a faculty committee on April 7, 2011. Results were disseminated at local and national conferences.

Thesis Mentor: Sandra Sephton, Ph.D.

Committee Members: Paul Salmon, Ph.D. and L. Kevin Chapman, Ph.D.

Undergraduate Research Assistant

May 2010-April 2011

Completed yearly progress reports and created IRB amendments for the laboratory's randomized controlled trial of meditation for fibromyalgia, and observational research among women with cancer. Updated literature and revised a manuscript for publication from the fibromyalgia RCT. Updated the literature, researched healthy cytokine levels, and updated the Endnote library for a breast cancer study. Updated literature and revised a manuscript for publication using gynecologic cancer data. An undergraduate research grant was written and submitted which proposes a study involving genotyping in newly diagnosed breast cancer patients.

Directed by Sandra Sephton, Ph.D.

Supervised by Elizabeth Lush, M.S. (Ph.D. Candidate)

Undergraduate Research Assistant

May 2010-April 2011

Managed regulatory issues, developed study documents, edited study materials, prepared and submitted materials for IRB approval and IRB amendments for an iPod-based stress reduction intervention in breast cancer patients. Completed human subjects protection 
training, biohazardous waste training, HIPAA training, and received credentials through University of Louisville Hospital to recruit patients from the surgical oncology clinics.

Directed by Sandra Sephton, Ph.D.

Undergraduate Research Assistant

January 2010-April 2010

Reviewed and formatted baseline, follow-up psychometric, and cortisol questionnaires, set up an SPSS database, drafted a paragraph for the study protocol regarding a newly added measure, and researched measures and articles regarding body image, sexual functioning, and relationships in women with breast cancer for the iPod-based stress reduction intervention in breast cancer study. Completed the yearly progress report and updated the literature for the lung cancer study, edited graphs for the Parkinson's disease study, researched the 5HTT, PER3, and NPAS2 genes, and collected /processed salivary cortisol data.

Directed by Sandra Sephton, Ph.D.

Supervised by Emily Eismann, B.A. (Ph.D. Candidate)

Undergraduate Research Assistant

September 2009-December 2009

Entered data into SPSS and cleaned the undergraduate stress study data.

Directed by Sandra Sephton, Ph.D.

Supervised by Scott Hanneman, B.A. (Ph.D. Candidate)

\section{Laboratory and Data Analytic Experience}

Endocrinology

Experience preparing and running enzyme-linked immunosorbent assays (ELISA) for salivary cortisol. Experience in collecting, preparing, processing, and preserving human saliva samples. Experience in cortisol data cleaning and reduction.

Immunology

Experience processing and preserving serum and plasma human blood samples.

Experience preparing under sterile technique and preserving human whole blood samples for stimulated immunomodulator analysis. Experience preparing immunomodulator stimulation media.

Circadian Rhythms

Experience in initializing and downloading MicroMini Motionlogger Actiwatches. Experience in cleaning and scoring actigraphy data.

Data Analysis

Experience in conducting univariate and multivariate analyses, structural equation modeling, hierarchical linear modeling, survival analyses, and geocoding. Experience using the following statistical software: SPSS, AMOS, HLM, and American Fact Finder (Census data). 


\section{Research Funding}

Title: Understanding the prognostic significance of circadian disruption in lung cancer, and piloting an intervention

Role: Research Assistant

PI: Sandra Sephton, Ph.D.

Status: Funded

Dates: June 1, 2014-May 31, 2016

Budget: $\$ 150,000$

Sponsor: Kentucky Lung Cancer Research Board

Description: This study will explore circadian disruption as a mediator of psychosocial effects on tumor progression. Innovations of the study include a focus on circadian disruption as a potential mediator of psychosocial effects on tumor progression; studying distress in a population of high need; and piloting a portable, highly feasible psychosocial intervention in lung cancer patients.

Title: Gene-Environment Interactions in the Biobehavioral Sequelae of Breast Cancer

Diagnosis and Treatment

Role: Research Assistant

PI: Sandra Sephton, Ph.D.

Status: Funded

Dates: January 1, 2011-June 30, 2011

Budget: $\$ 3,000$

Sponsor: University of Louisville Intramural Incentives Grant-Undergraduate Research Grant

Description: This genotyping study will explore how the serotonin transporter gene (5HTT) and the circadian genes Period3 (PER3) and Neuronal PAS domain protein 2 (NPAS2) interact with the stress of breast cancer diagnosis and treatment to produce psychopathology and circadian disruption.

\section{Published Manuscripts}

Rebholz, W.N.; Cash, E.; Zimmaro, L.A.; Bayley-Veloso, R.; Phillips, K.; Chagpar, A.; Dhabhar, F.; Spiegel, D.; Saltsman Bell, B.N.; Sephton, S.E. (2016) Distress and QOL in an Ethnically Diverse Sample Awaiting Breast Cancer Surgery. Journal of Health Psychology. In Press. doi: 10.1177/1359105316659916.

Zimmaro, L.A., Salmon, P., Naidu, H., Rowe, J., Phillips, K., Rebholz, W.N., GieseDavis, J., Cash, E., Dreeben, S., Bayley-Veloso, R.C., Jablonski, M., Hicks, A., Siwik, C., and Sephton, S.E. (2016) The Role of Dispositional Mindfulness in Stress and Health Outcomes in University Undergraduate Students. Mindfulness, 7, 874-885. doi: 10.1007/s12671-016-0526-8.

Cash, E.; Sephton, S.E.; Chagpar, A.B.; Spiegel, D.; Rebholz, W.N.; Zimmaro, L.A.; Tillie, J.M.; Dhabhar, F.S. (2015) Circadian disruption and tumor promotion/progression signals in breast cancer patients awaiting surgery. Brain Behavior, Immunity, 102-114. doi: 10.1016/j.bbi.2015.02.017. 
Cash, E.; Salmon, P.; Weissbecker, I.; Rebholz, W.N; Bayley-Veloso, R.; Zimmaro, L.; Floyd, A.; Dedert, E.; Sephton, S.E. (2014). Mindfulness Meditation Alleviates Fibromyalgia Symptoms in Women: Results of a Randomized Trial. Annals of behavioral medicine, 1-12. doi: 10.1007/s12160-014-9665-0

Sephton, S. E., Lush, E., Dedert, E. A., Floyd, A. R., Rebholz, W. N., Dhabhar, F. S., Spiegel, D., \& Salmon, P. (2013). Diurnal cortisol rhythm as a predictor of lung cancer survival. Brain, behavior, and immunity, 30 Suppl, S163-170. doi: 10.1016/j.bbi.2012.07.019

\section{Published Abstracts and Presentations}

Seibert, A.; Pace, A.; Rebholz, W.; Wilson, L.; Bumpous, J.M.; Cash, E. (2016). Preoperative Biomarkers as Predictors of Surgical Complications and Survival in Head and Neck Cancer. Poster presentation at the AHNS $9^{\text {th }}$ Annual International Conference, Seattle, WA.

Pace, A.; Seibert, A.; Rebholz, W.; Wilson, L.; Bumpous, J.M.; Cash, E. (2016). Psychological Distress and Malnutrition Biomarkers are associated with Head and Neck Cancer Progression and Survival. Oral paper presentation at the American Psychosomatic Society $74^{\text {th }}$ Annual Scientific Meeting, Denver, CO.

Rebholz, W.N., Weissbecker, I., Cash, E., Bayley-Veloso, R., Zimmaro, L.A., Phillips, K., Sephton, S.E. (2015). Diurnal Cortisol Rhythms and Systemic Norepinephrine Predict Gynecologic Cancer Survival. Poster presentation at the American Psychosomatic Society 73rd Annual Scientific Conference, Savannah, GA.

Cash, E.; Chilton, P.; Rebholz, W.; Bayley-Veloso, R.; Zimmaro, L.; Chagpar, A.B.; Spiegel, D.; Dhabhar, F.S.; Sephton, S.E. (2015). HPA and Rest/Activity Rhythms Independently Associated with Different Aspects of Inflammatory Response in Patients Awaiting Treatment for Breast Cancer. Oral paper presentation at the American Psychosomatic Society 73rd Annual Scientific Meeting, Savannah, GA.

Bayley-Veloso, R., Weissbecker, I., Rebholz, W.N., Cash, E., Zimmaro, L.A., Phillips, K., Salmon, P., Sephton, S.E. (2015). Do traumatic events build resilience and promote survival through social support in gynecologic cancer?. Poster presentation at the American Psychosomatic Society 73rd Annual Scientific Conference, Savannah, GA.

Zimmaro, L.A.; Rebholz, W.; Cash, E.; Bayley-Veloso, R.; Phillips, K.; Salmon, P.; Sephton, S.E. (2105). Optimism as a Moderator of Psychoneuroimmune Pathways in Lung Cancer Patients. Poster presentation at the American Psychosomatic Society 73rd Annual Scientific Conference, Savannah, GA.

Phillips, K.; Salmon, P.; Patel, H.; Rowe, J.; Rebholz, W.; Zimmaro, L.; Bayley-Veloso, R.; Cash, E.; Giese-Davis, J.; Sonnier, H.; Sephton, S.E. (2015). The Role of 
Mindfulness in Stress and Health Outcomes in University Undergraduate Students. Poster presentation at the American Psychosomatic Society 73rd Annual Scientific Conference, Savannah, GA.

Gettelfinger, J.; Rebholz, W.; Russ, E.; Wilson, L.; Sephton, S.E.; Bumpous, J.M.; Cash, E. (2014). Depressive Symptoms Predict Poor Treatment Adherence and Response in Head and Neck Cancer. Paper presentation at the Kentucky Society for Otolaryngology Annual Meeting, August 1-2.

Cash, E.; Gettelfinger, J.; Rebholz, W.; Russ, E.; Wilson, L.; Sephton, S.E.; Bumpous, J.M. (2014). Depressive Symptoms Predict Poor Treatment Adherence and Response in Head and Neck Cancer. Paper presentation at the International Federation of Head and Neck Oncologic Societies $5^{\text {th }}$ World Congress and American Head and Neck Society Annual Meeting, New York, NY, July 26-30.

Rebholz, W.N., Weissbecker, I., Cash, E., Bayley, R., Zimmaro, L.A., Sephton, S.E. (2014). Distress and support: Links with circadian disruption and quality of life in gynecologic cancer. Oral paper presentation at the American Psychosomatic Society 72nd Annual Scientific Conference, San Francisco, CA.

Sephton, S.E., Cash, E., Chagpar, A., Spiegel, D., Rebholz, W.N., Bayley, R.C., Zimmaro, L.A., and Dhabhar, F.S. (2014). Biological correlates of marital status in recently diagnosed breast cancer patients. Oral paper presentation at the American Psychosomatic Society 72nd Annual Scientific Conference, San Francisco, CA.

Cash, L., Rebholz, W., Gettelfinger, J., Russ, E., Wilson, L., Bumpous, J. M., Sephton, S. E. (2014). Depressive Symptoms Predict Poor Treatment Adherence and Response in Head and Neck Cancer. Poster presentation at the American Psychosomatic Society 72nd Annual Scientific Conference, San Francisco, CA.

Bayley, R., Rebholz, W., Cash, E., Weissbecker, I., Salmon, P., Sephton, S. (2014). Associations between Trauma Across the Lifespan, Health Behaviors, and Allostatic Load in Gynecologic Cancer Patients. Poster presentation at the American Psychosomatic Society 72nd Annual Scientific Conference, San Francisco, CA.

Zimmaro, L.A., Rebholz, W.N., Bayley, R., Cash, L., Sephton, S.E. (2014). Gender Differences among Lung Cancer Patients in Appraisal, Coping, and Distress. Poster presentation at the American Psychosomatic Society 72nd Annual Scientific Conference, San Francisco, CA.

Rebholz, W.N., Cash, E., Dhabhar, F., Chagpar, A., Chapman, L.K., Sephton, S.E. (2013). Psychological distress, but not stress physiology, is associated with quality of life in presurgical breast cancer patients. Poster presentation at the 
American Psychosomatic Society 71st Annual Scientific Conference, Miami, Florida.

Rebholz, W.N., Sephton, S. (2013). Social support in lung cancer patients: the association with endocrine and immune function. Poster presentation at the American Psychosomatic Society 71st Annual Scientific Conference, Miami, Florida.

Cash, E., Sephton, S.E., Chagpar, A.B., Spiegel, D., Rebholz, W.N., Dhabhar, F.S. (2013). Circadian disruption and tumor promotion/progression signals in newly diagnosed breast cancer patients. Poster presentation at the American Psychosomatic Society 71st Annual Scientific Conference, Miami, Florida.

Bayley, R., Rebholz, W.N., Sephton, S.E. (2013). Cancer-specific distress, coping, and health behaviors in lung cancer patients. Poster presentation at the American Psychosomatic Society 71st Annual Scientific Conference, Miami, Florida.

Gesler, W., Lush, E., Dhabhar, F., Chagpar, A., Chapman, L.K., Dedert, E., Sephton, S. (2012). Psychological distress, but not stress physiology, is associated with quality of life in presurgical breast cancer patients. Poster presentation at the University of Louisville Graduate Student Research Symposium, Louisville, KY.

Gesler, W.N.; Dhabhar, F.S.; Chagpar, A.B.; Lush, E.; Dedert, E.A.; Daup, M.B.; Spiegel, D.; Dayyat, E.A.; Tillie, J.M.; McMasters, K.M.; Sephton, S.E. (2011). Cancer-Specific Distress: No Association with Serum Cytokines in Presurgical Breast Cancer Patients. Poster presentation at the American Psychosomatic Society 69th Annual Scientific Conference, San Antonio, TX.

Daup, M., Lush, E., Gesler, W., Dedert, E., McMasters, K., Sephton, S., Chagpar, A. (2010). Is cancer specific distress correlated with time to definitive surgery in breast cancer patients? Poster Presentation at the San Antonio Breast Cancer Symposium, San Antonio, TX.

Gesler, W., Dhabhar, F., Chagpar, A., Dedert, E., Lush, E., Daup, M., Dayyat, E., McMasters, K., Sephton, S.E. (2010). Distress and Inflammation in Women Newly Diagnosed with Breast Cancer. Poster Presentation at the James Graham Brown Cancer Center Ninth Annual Retreat, Louisville, KY.

\section{Manuscripts in Preparation}

Rebholz, W.N.; Cash, E.; Bayley, R.C.; Zimmaro, L.A.; Phillips, K.; Siwik, C.; Sephton, S.E. Diurnal Cortisol Rhythm and Norepinephrine Predict Gynecologic Cancer Survival. To be submitted to Journal of the National Cancer Institute.

Jablonski, M. E.; Dreeben, S.; Hanneman, S.; Vines, L.; Rebholz, W.N.; Bayley, R.; Eismann, E.; Sephton, S.E.; Litvan, I.; Houghton, D.; Constantino, A.; Salmon, P. A Randomized-Controlled Pilot Study of Mindfulness-Based Stress Reduction (MBSR) for Early-Stage Parkinson's Disease Patient and Caregiver Dyads. 
Rebholz, W.N.; Weissbecker, I.; Cash, E.; Bayley, R.C.; Sephton, S.E. Distress and Support: Links with Circadian Disruption and Quality of Life in Gynecologic Cancer. To be submitted to Psychoneuroendocrinology.

Bayley, R., Rebholz, W.N., Cash, E., Weissbecker, I., Salmon, P., Sephton, S. Associations between Trauma Across the Lifespan, Health Behaviors, and Allostatic Load in Gynecologic Cancer Patients.

Sephton, S.E.; Cash, E.; Chagpar, A.B.; Spiegel, D.; Rebholz, W.N.; Bayley, R.C.; Zimmaro, L.Z.; Dhabhar, F.S. Breast Cancer, Circadian Disruption, and Stimulated Immunity. To be submitted to Brain, Behavior, Immunity.

Rebholz, W.N.; Sephton, S.E. The Effects of Coping and Social Support on Physiology in Patients with Lung Cancer.

Zimmaro, L.A.; Cash, E.; Rebholz, W.N.; Bayley, R.C.; Sephton, S.E. Mindfulness, Sleep, and Circadian Rhythms.

\section{Clinical Experience}

July 2015-July 2016

Predoctoral clinical psychology internship at the Central Texas Veterans Health Care System. The predoctoral clinical psychology internship includes three major rotations, which are four days a week for three months each, two minor rotations, which are one half day a week for six months, and a half day of didactics/group supervision a week. Major rotations included primary care/behavioral health, health behavior clinic, and mental health clinic. Minor rotations included the psychosocial rehabilitation resource center and the Post-Traumatic Stress Disorder clinic. Additional activities included case conference write-up and presentation (assessment and intervention case), didactics (EBP and general topics relevant to clinical psychology and working in the VA), group supervision, diversity seminar, interdisciplinary professional training presentations, and staff meetings.

Training Directors: Andrew Cook, Ph.D. and Erin Andrews, Psy.D., ABPP

April 2016-July 2016

Predoctoral clinical psychology internship

The mental health clinic major rotation includes consultation intakes, individual therapy, group therapy (facilitating acceptance and commitment therapy (ACT) for anxiety and depression and co-facilitating cognitive processing therapy (CPT) for sexual trauma), treatment planning, consultation, staff meetings, chart review/clinical report writing, reading, and one-on-one supervision.

Supervised by Kentrell Avery, Psy.D. 
February 2016-July 2016

Predoctoral clinical psychology internship

The Post-Traumatic Stress Disorder clinic minor rotation includes individual therapy (coping skills for PTSD, CPT, and prolonged exposure), treatment planning, chart review/clinical report writing, reading, and one-on-one supervision.

Supervised by Regina Hiraoka, Ph.D.

November 2015-March 2016

Predoctoral clinical psychology internship

The health behavior clinic major rotation included presurgical evaluations (transplant and bariatric), consultation intakes and individual therapy (CBT-insomnia, CBT-chronic pain, and chronic disease management), co-facilitating group therapy (MOVE weight management, CBT-insomnia, tobacco cessation, disease management), treatment planning, consultation, national conference calls, case conferences and presentation, staff meetings, co-facilitating motivational interviewing and TEACH trainings, audio recordings, chart review/clinical report writing, reading, and one-on-one supervision. Supervised by Andrew Cook, Ph.D.

July 2015-January 2016

Predoctoral clinical psychology internship

The psychosocial resource and recovery center minor rotation included treatment planning sessions, recovery coaching, individual therapy, co-facilitating group therapy (mindfulness for depression and stress management), treatment planning, staff meetings, audio recording, chart review/clinical report writing, reading, and one-on-one supervision. This clinic population includes Veterans with long-term severe mental illness needs.

Supervised by Jennifer Rigsby, Ph.D.

July 2015-November 2015

Predoctoral clinical psychology internship

The primary care/behavioral health major rotation included consultation intakes, brief solution-focused therapy, consultation-liaison, co-facilitating group therapy (CBTinsomnia, CBT-chronic pain, and mindfulness-based stress reduction), staff meetings, audio recording, chart review/clinical report writing, reading, and one-on-one supervision.

Supervised by Claudia Goleburn, Psy.D.

August 2014-June 2015

Graduate program practicum

Clinical practicum learning Cognitive-Behavioral therapeutic interventions with a focus on treatment of anxiety disorders in adults and children. Weekly team supervision, peer consultation, one-on-one supervision, intakes, assessments, individual therapy, group therapy, case conceptualization, audio/visual digital recording review, chart review/clinical report writing, and reading are activities that are included.

Supervised by Janet Woodruff-Borden, Ph.D. 
May 2014-June 2015

Clinic Graduate Teaching Assistant

Graduate teaching assistant at the Noble H. Kelley Psychological Services Center, a graduate student run clinic at the University of Louisville. Responsibilities include coordinating the PSC colloquium series, opening and closing the clinic, checking in clients for appointments, phone intakes for therapy and assessment clients, clinical interviews/intakes with prospective therapy clients; supervision of graduate students; one-on-one supervision, chart review, report writing, and audio/visual digital recording review are activities that are included.

Supervised by Bernadette Walter, Ph.D.

April 2014-June 2015

Graduate program practicum

Neuropsychology practicum learning how to conduct dementia assessments. Clinical interviewing, administration of neuropsychological assessment battery, collateral interview, one-on-one supervision, chart review/assessment report writing, and reading are all activities that are included. Additional opportunities to complete surgical evaluations (i.e., cochlear implant), conduct cognitive assessments in children with possible central auditory processing disorder, and work with therapy clients are included. Supervised by Elizabeth Cash, Ph.D. and Eric Russ, Ph.D.

August 2013-August 2014

Graduate program practicum

Clinical practicum learning Behavioral/Cognitive-Behavioral therapeutic interventions with a focus on treatment of ADHD and emotional difficulties in children. Weekly team supervision, peer consultation, one-on-one supervision, intakes, assessments, individual therapy, group therapy, case conceptualization, audio/visual digital recording review, chart review/clinical report writing, and reading are activities that are included. Supervised by Paul Rosen, Ph.D.

May 2013-May 2014

Graduate program practicum

Head and neck cancer clinic practicum learning how to conduct health and behavior assessments and medical/health related interventions for newly diagnosed patients. Clinical interviewing, administration of self-report questionnaires, consultation/liaison work, evaluations (e.g., cochlear implant), one-on-one supervision, chart review/assessment report writing, program development, and reading are activities that are included.

Supervised by Elizabeth Cash, Ph.D. and Eric Russ, Ph.D.

May 2012-June 2015

Graduate program practicum

Assessment practicum learning how to conduct ADHD and learning disability testing for adults. Clinical interviewing, administration of standardized testing, one-on-one supervision, chart review/clinical report writing, and reading are activities that are included. 
Supervised by David Winsch, Ph.D.

May 2012-June 2015

Graduate program practicum

Assessment practicum learning how to conduct advanced placement testing for children. Clinical interviewing of parents/legal guardians, administration of standardized testing, one-on-one supervision, chart review/clinical report writing, and reading are activities that are included.

Supervised by Bernadette Walter, Ph.D.

August 2011-August 2013

Graduate program practicum

Clinical practicum learning a Mindfulness approach to therapeutic interventions, primarily working with adults with mood disorders. Weekly team supervision, peer consultation, one-on-one supervision, individual therapy, case conceptualization, audio/visual digital recording review, chart review/clinical report writing, and reading are activities that are included.

Supervised by Paul Salmon, Ph.D.

\section{Teaching Experience}

2011-2015

Graduate Teaching Assistant, University of Louisville Department of Psychological and Brain Sciences

Courses Assisted: Psychology 385: Abnormal Psychology; Psychology 344:

Physiological Psychology; Psychology 443: Intro to Neuroscience; Psychology 302:

Experimental Psychology (including teaching recitations); Psychology 372: Social

Psychology; Psychology 375: Personality; Psychology 693: Interviewing Skills

Practicum; Psychology 680: Intellectual and Cognitive Assessment

\section{Community Service \& Volunteer Experience}

Peer Reviewer: American Psychosomatic Society Conference Abstracts

Athena's Sisters Empowerment Workshop

"I am woman, hear me roar"

Peer Reviewer: American Psychosomatic Society

Conference Abstracts

Psychiatry Resident Lecture

Topic: Geriatric Neuropsychological Assessment

Peer Reviewer: Psychosomatic Medicine

Peer Reviewer: Psycho-Oncology
November 2015

Louisville, $\mathrm{KY}$

May 11, 2015

November 2014

Louisville, KY

October 2014

October 2014

June 2014 
Cohort Liaison to the Faculty

Dress for Success Talk

Topic: Stress Management and Your Career

Dress for Success Health Fair

Psychological Services Center Representative

Heart Walk Volunteer

American Heart Association

St. James Catholic Church

Volunteer

University of Louisville

Disability Resource Center Note Taker

Children's Hospital Foundation

Teen Advisory Board President

National Youth Leadership Forum

Medical Outreach Program

Professional Membership

APA Division 29- Psychotherapy

APA Division 38- Health Psychology

American Psychological Association

APAGS-Campus Representative

Kentucky Psychological Association

American Psychosomatic Society

Psi Chi International Honor Society in Psychology

Vice President and Historian: UofL Chapter Fundraising Coordinator: UofL Chapter
October 2013-September 2014

Louisville, KY

June 18, 2013

Louisville, KY

November 10, 2012

Louisville, $\mathrm{KY}$

September 22, 2012

Louisville, KY

Winter 2011

Louisville, KY

Fall 2010

Louisville, KY

Summer 2006-Spring 2007

Atlanta, GA

July 25-29, 2006
Summer 2013-Summer 2014

Summer 2013-Present

Summer 2011-Present

August 2013-August 2014

Summer 2011-Present

Spring 2011-Present

Inducted Spring 2010

Fall 2010-Summer 2011

Fall 2009-Summer 2010 